\title{
Forecasting of work in process quality using Holt-Winters method for missing observations
}

\author{
Sarang Ram Kayande \\ West Virginia University
}

Follow this and additional works at: https://researchrepository.wvu.edu/etd

\section{Recommended Citation}

Kayande, Sarang Ram, "Forecasting of work in process quality using Holt-Winters method for missing observations" (1999). Graduate Theses, Dissertations, and Problem Reports. 972.

https://researchrepository.wvu.edu/etd/972

This Thesis is protected by copyright and/or related rights. It has been brought to you by the The Research Repository @ WVU with permission from the rights-holder(s). You are free to use this Thesis in any way that is permitted by the copyright and related rights legislation that applies to your use. For other uses you must obtain permission from the rights-holder(s) directly, unless additional rights are indicated by a Creative Commons license in the record and/ or on the work itself. This Thesis has been accepted for inclusion in WVU Graduate Theses, Dissertations, and Problem Reports collection by an authorized administrator of The Research Repository @ WVU. For more information, please contact researchrepository@mail.wvu.edu. 


\title{
FORECASTING OF WORK IN PROCESS QUALITY USING HOLT-WINTERS METHOD FOR MISSING OBSERVATIONS
}

Sarang R. Kayande

\author{
Thesis Submitted to the \\ College of Engineering and Mineral Resources \\ At West Virginia University \\ In Partial fulfillment of the Requirements for \\ the Degree of \\ Master of Science
in
Industrial Engineering
}

Dr. Majid Jaraiedi, Ph.D., Chair

Dr. B. Gopalakrishnan, Ph.D.

Dr. Ralph Plummer, Ph.D.

Department of Industrial and Management Systems Engineering

\author{
Morgantown, West Virginia
}

1999

Keywords: Missing Observations, Holt-Winters Method, Forecasting 


\section{ABSTRACT \\ Forecasting of Work in Process Quality \\ Using Holt-Winters Method for Missing Observations}

\section{Sarang R. Kayande}

Since the pace of technological change is so great, and since new products and processes may be key to a company's future plans, an increasing number of companies are emphasizing regular and complete technological forecasts affecting their industry. Those companies which have gone far in developing planning premises from their technological forecasts have tended to be high-technology enterprises. What has been done in these instances is to encourage members of their technical staffs to be alert to future developments; to think in terms of the impact of current scientific developments on the future state of technology; and to develop orderly forecasts of how these developments affect the company's products, processes or markets. Many attempts have been made to accurately forecast future and some of the accurate and meaningful methods used to forecast the state of technology are the Delphi technique, the opportunity and goal oriented techniques.

The objective of this research was to develop a forecasting model using extension of Holt-Winters method for missing data. The variable of interest considered was the fraction non-conforming of a process. Initial values were generated using Beta distribution. Values of fraction non-conforming for future periods were generated using different processes such as Autoregressive and Autoregressive Moving Average process. Some of the values in each data set were assumed to be missing. The factors that were considered for forecasting using this method were the level, trend and the seasonal factor. Forecasting was done for at least one period ahead and at the most twelve periods ahead. The developed models were found to give acceptable results with as many as $40 \%$ of the total observations missing and this was validated by performing tracking signal analysis. 


\section{ACKNOWLEDGEMENTS}

I would like to express my sincere thanks and gratitude to Dr. Majid Jaraiedi, my committee chair for his continuous support through the research. He was always available to answer my questions and explicate problems that I encountered.

I also appreciate the time and efforts of Dr. B. Gopalakrishnan, my committee member who offered numerous constructive suggestions. Further, I would like to thank Dr. Ralph Plummer for his constant encouragement and support throughout the research.

Finally, I would like to thank my parents, my better half to be and all my friends for their support all through my studies. I dedicate this thesis to my grandmother. 


\section{TABLE OF CONTENTS}

ABSTRACT.............................................. ii

ACKNOWLEDGEMENT ................................. iii

TABLE OF CONTENTS...................................... iv

LIST OF FIGURES....................................... vii

LIST OF TABLES...................................... ix

1. Introduction and Research Objectives.................... 1

1.1 Background 1

1.2 Defining the Forecasting Problem 3

1.3 Time Series Models 4

1.4 Model Selection 6

1.5 Need for Research 8

1.6 Research Objectives 9

$\begin{array}{lll}1.7 & \text { Organization } & 10\end{array}$

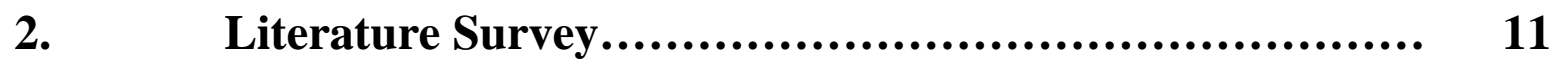

2.1 Linear Filter Model 11

2.1.1 Autoregressive Process 12

2.2 Moving Average Models 14

2.2.1 Mixed Autoregressive-Moving Average Models 14 
$\begin{array}{lll}2.3 .1 & \text { Parsimony } & 18\end{array}$

2.4 Exponential Smoothing for Irregularly Spaced Data 20

2.5 Holt's Method for Regularly Spaced Data 21

2.6 Extension of Holt-Winters Method 24

2.7 Tracking Signal Tests 28

2.7.1 Tests Based on the Cumulative Forecast Error $\quad 29$

2.7.2 Tests Based on the Smoothed Forecast Error $\quad 30$

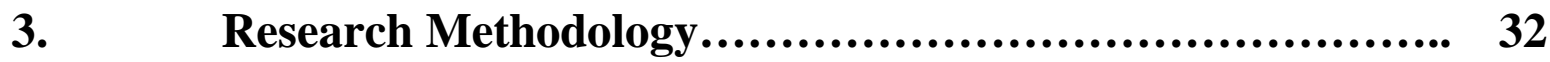

3.1 Assumptions 32

3.2 Symbols and Notations 33

3.3 Calculation of the Initial Values 34

3.4 Method Adopted to Develop the Model 35

3.4.1 Data Generation and Model Development 35

3.4.2 Tracking Signal Analysis 37

Example $1 \quad 38$

3.4.3 Analysis of the Developed Model 45

4. Analysis of Results............................................ 46

4.1 Results for AR(1) Process 47 
4.2 Results for AR(2) Process $\quad 50$

5. Conclusions and Future Research........................... 87

$\begin{array}{lll}5.1 \text { Conclusions } & 87\end{array}$

5.2 Future Research 88

REFERENCES............................................ 90

APPENDIX............................................... 92

APPROVAL OF EXAMINING COMMITTEE............... 


\section{LIST OF FIGURES}

$\begin{array}{lll}\text { Figure } & \text { Description } & \text { Pg. No }\end{array}$

$\begin{array}{llr}2.1 & \text { Linear Filter } & 12\end{array}$

$\begin{array}{lll}2.2 & \text { Block Diagram for ARIMA Model } & 16\end{array}$

3.1 Generated vs. Predicted Values, AR(1) Process, 12 Observations Missing 43

3.2 Generated vs. Forecasted Values, AR(1) Process, 12 Observations Missing 43

4.1.1(a) Generated vs. Predicted Values, AR(1) Process, 12 Observations Missing 52

4.1.1(b) Generated vs. Forecasted Values, AR(1) Process, 12 Observations Missing 52

4.1.2(a) Generated vs. Predicted Values, AR(1) Process, 16 Observations Missing 54

4.1.2(b) Generated vs. Forecasted Values, AR(1) Process, 16 Observations Missing 54

4.1.3(a) Generated vs. Predicted Values, AR(1) Process, 18 Observations Missing 56

4.1.3(b) Generated vs. Forecasted Values, AR(1) Process, 18 Observations Missing 56

4.2.1(a) Generated vs. Predicted Values, AR(2) Process, 11 Observations Missing 58

4.2.1(b) Generated vs. Forecasted Values, AR(2) Process, 11 Observations Missing 58

4.2.2(a) Generated vs. Predicted Values, AR(2) Process, 13 Observations Missing 60

4.2.2(b) Generated vs. Forecasted Values, AR(2) Process, 13 Observations Missing 60

4.2.3(a) Generated vs. Predicted Values, AR(2) Process, 16 Observations Missing 62

4.2.3(b) Generated vs. Forecasted Values, AR(2) Process, 16 Observations Missing 62

4.2.4(a) Generated vs. Predicted Values, AR(2) Process, 18 Observations Missing 64

4.2.4(b) Generated vs. Forecasted Values, AR(2) Process, 18 Observations Missing 64

4.3.1(a) Generated vs. Predicted Values, ARMA(1,1) Process, 10 Observations Missing 66

4.3.2(a) Generated vs. Predicted Values, ARMA(1,1) Process, 11 Observations Missing 68

4.3.2(b) Generated vs. Forecasted Values, ARMA(1,1) Process, 11 Observations Missing 68 
4.3.3(a) Generated vs. Predicted Values, ARMA(1,1) Process, 13 Observations Missing 70

4.3.3(b) Generated vs. Forecasted Values, ARMA(1,1) Process, 13 Observations Missing $\quad 70$

4.3.4(a) Generated vs. Predicted Values, ARMA(1,1) Process, 18 Observations Missing 72

4.3.4(b) Generated vs. Forecasted Values, ARMA(1,1) Process, 18 Observations Missing 72

4.4.1(a) Generated vs. Predicted Values, ARMA(1,2) Process, 11 Observations Missing 74

4.4.1(b) Generated vs. Forecasted Values, ARMA(1,2) Process, 11 Observations Missing 74

4.4.2(a) Generated vs. Predicted Values, ARMA(1,2) Process, 15 Observations Missing 76

4.4.2(b) Generated vs. Forecasted Values, ARMA(1,2) Process, 15 Observations Missing 76

4.4.3(a) Generated vs. Predicted Values, ARMA(1,2) Process, 17 Observations Missing 78

4.4.3(b) Generated vs. Forecasted Values, ARMA(1,2) Process, 17 Observations Missing 78

4.4.4(a) Generated vs. Predicted Values, ARMA(1,2) Process, 18 Observations Missing 80

4.4.4(b) Generated vs. Forecasted Values, ARMA(1,2) Process, 18 Observations Missing $\quad 80$

4.5.1(a) Generated vs. Predicted Values, ARMA(2,2) Process, 11 Observations Missing 82

4.5.1(b) Generated vs. Forecasted Values, ARMA(2,2) Process, 11 Observations Missing 82

4.5.2(a) Generated vs. Predicted Values, ARMA(2,2) Process, 14 Observations Missing 84

4.5.2(b) Generated vs. Forecasted Values, ARMA(2,2) Process, 14 Observations Missing 84

4.5.3(a) Generated vs. Predicted Values, ARMA(2,2) Process, 12 Observations Missing 86

4.5.3(b) Generated vs. Forecasted Values, ARMA(2,2) Process, 12 Observations Missing 86 


\section{LIST OF TABLES}

3.1 Forecasting fraction defective for AR(1) process, 12 observations missing 42

3.2 Tracking signal analysis $\{$ AR(1) process, 12 observations missing $\} \quad 44$

4.1.1 Forecasting fraction defective for AR(1) process, 12 observations missing 51

4.1.2 Forecasting fraction defective for AR(1) process, 16 observations missing 53

4.1.3 Forecasting fraction defective for AR(1) process, 18 observations missing 55

4.2.1 Forecasting fraction defective for AR(2) process, 11 observations missing $\quad 57$

4.2.2 Forecasting fraction defective for AR(2) process, 13 observations missing 59

4.2.3 Forecasting fraction defective for AR(2) process, 16 observations missing 61

4.2.4 Forecasting fraction defective for AR(2) process, 18 observations missing 63

4.3.1 Forecasting fraction defective for $\operatorname{ARMA}(1,1)$ process, 10 observations missing 65

4.3.2 Forecasting fraction defective for $\operatorname{ARMA}(1,1)$ process, 11 observations missing $\quad 67$

4.3.3 Forecasting fraction defective for $\operatorname{ARMA}(1,1)$ process, 13 observations missing 69

4.3.4 Forecasting fraction defective for $\operatorname{ARMA}(1,1)$ process, 18 observations missing 71

4.4.1 Forecasting fraction defective for $\operatorname{ARMA}(1,2)$ process, 11 observations missing 73

4.4.2 Forecasting fraction defective for $\operatorname{ARMA}(1,2)$ process, 15 observations missing 75

4.4.3 Forecasting fraction defective for $\operatorname{ARMA}(1,2)$ process, 17 observations missing 77

4.4.4 Forecasting fraction defective for $\operatorname{ARMA}(1,2)$ process, 18 observations missing 79

4.5.1 Forecasting fraction defective for $\operatorname{ARMA}(2,2)$ process, 11 observations missing 81

4.5.2 Forecasting fraction defective for $\operatorname{ARMA}(2,2)$ process, 14 observations missing 83

4.5.3 Forecasting fraction defective for $\operatorname{ARMA}(2,2)$ process, 12 observations missing 85

A.1.1 Tracking signal analysis $\{$ AR(1) process, 12 observations missing $\} \quad 92$

A.1.2 Tracking signal analysis \{AR(1) process, 16 observations missing $\} \quad 93$ 
A.1.3 Tracking signal analysis \{AR(1) process, 18 observations missing \} 94

A.2.1 Tracking signal analysis \{AR(2) process, 11 observations missing $\} \quad 95$

A.2.2 Tracking signal analysis \{AR(2) process, 13 observations missing $\} \quad 96$

A.2.3 Tracking signal analysis \{AR(2) process, 16 observations missing $\} \quad 97$

A.2.4 Tracking signal analysis $\{\mathrm{AR}(2)$ process, 18 observations missing $\} \quad 98$

A.3.1 Tracking signal analysis $\{$ ARMA(1,1) process, 10 observations missing $\} \quad 99$

A.3.2 Tracking signal analysis \{ARMA(1,1) process, 11 observations missing $\} \quad 100$

A.3.3 Tracking signal analysis $\{$ ARMA(1,1) process, 13 observations missing $\} 101$

A.3.4 Tracking signal analysis \{ARMA(1,1) process, 18 observations missing $\} 102$

A.4.1 Tracking signal analysis $\{\operatorname{ARMA}(1,2)$ process, 11 observations missing $\} 103$

A.4.2 Tracking signal analysis \{ARMA(1,2) process, 15 observations missing $\} 104$

A.4.3 Tracking signal analysis \{ARMA(1,2) process, 17 observations missing $\} 105$

A.4.4 Tracking signal analysis $\{\operatorname{ARMA}(1,2)$ process, 18 observations missing $\} 106$

A.5.1 Tracking signal analysis $\{$ ARMA(2,1) process, 12 observations missing $\} 107$

A.6.1 Tracking signal analysis $\{\operatorname{ARMA}(2,2)$ process, 11 observations missing $\} \quad 108$

A.6.2 Tracking signal analysis $\{\operatorname{ARMA}(2,2)$ process, 14 observations missing $\} \quad 109$

A.6.3 Tracking signal analysis $\{\operatorname{ARMA}(2,1)$ process, 12 observations missing $\} \quad 110$

$\begin{array}{lll}\text { A.7 } & \text { Factors for computing prediction intervals } & 111\end{array}$ 


\section{INTRODUCTION AND RESEARCH OBJECTIVES}

\subsection{BACKGROUND}

One of the principal activities for improving productivity in manufacturing systems is to forecast the product defects during processes and to recover the processes. The product defects come from typically even small changes during manufacturing processes that cause big variations in the product that in turn result in out of acceptable limits. It is therefore necessary to forecast the product quality during processes and finding desirable process conditions that result in high yield. Forecasting the in-process product quality requires a predictive model that identifies the relationship between a set of productivity measures and a set of process variables. Casual models are generally based on multiple regression. However, since the forecasting difficulty grows exponentially with the number of predictors, it is necessary to have various techniques so as to reduce the number of variables. Further the accuracy of forecasting results is truly dependant on the determination of the true nature of the relationships between the productivity measures (or "predictee") and the process variables (or "predictor"). It is also important to know as to how well the model accounts for real manufacturing processes. So a casual forecasting model based on multiple regression needs to be developed to predict work-in-process quality under the current process conditions.

Much of the statistical methodology is concerned with models in which the observations are assumed to vary independently. In many applications dependence between the observations is regarded as a nuisance, and in planned experiments, 
randomization of the experimental design is introduced to validate analysis conducted as if the observations were independent. However a great deal of data occur in the form of time series where observations are dependent and where the nature of this dependence is of interest in itself. The body of techniques available for the analysis of such series of dependent observations is time series analysis.

Time series are best represented by nonstationery models in which trends and other pseudo-systematic characteristic, which can change with time, are treated as statistical rather than as deterministic phenomena. Furthermore, some time series often posses marked seasonal or periodic components themselves capable of changing and needing seasonal statistical models for their description.

The process of model building is concerned with relating a class of statistical models to the data at hand and involves much more than model fitting. Thus, identification techniques, designed to suggest what particular kind of model might be worth considering, need to be developed first. The fitting of the identified model to a time series using likelihood function can then supply maximum likelihood estimates of the parameters. When accuracy of forecasts is the objective, the fitted statistical model is used directly to generate optimal forecasts by simple recursive calculation. In particular, this model completely determines whether the forecast projections should follow a straight line, an exponential curve, and so on. In addition, the fitted model allows one to see exactly how the forecasts utilize past data, to determine the variance of the forecast errors, and to calculate limits within which a future value of the series will lie with a given probability. 


\subsection{DEFINING THE FORECASTING PROBLEM}

The use of available observations from a time series to forecast its value at some future time can provide a basis for planning, control, and optimization of industrial processes. Defining a forecasting problem starts with the decision problem. Information from the forecasting process is to be used to improve the decision process. In determining what is to be forecast, it is necessary to define the variables that are to be analyzed and predicted. The level of detail required is also an important consideration. Various factors influence the level of detail used: availability of data, accuracy attainable, cost of analysis, etc. In cases where the appropriate choice of variables is not clear, it is necessary to try several alternatives and select the one giving the best performance. This is generally done during the development of the forecasting system through simulation using historical data.

Another important class of decision involves elements such as the forecasting period, the forecasting horizon, and the forecasting interval. The forecasting period and horizon are usually dictated by the decision process requiring the forecast. For a forecast to be of value, the horizon must be no less than the lead-time for implementing the decision. The forecasting interval is determined by the operating mode of the data processing system that provides information on the variable being forecasted.

One more aspect of the forecasting problem relates to the required form of the forecast. It is generally convenient to conceive the variable of interest as being a random variable having a particular probability distribution. The decision problem requires an estimate of some characteristic of the distribution, such as the mean, median, or most 
probable value for use as a forecast of the variable. Usually the forecast demands an estimate of the expected value of the variable, plus an estimate of the standard deviation of the forecast error.

\subsection{TIME SERIES MODELS}

A time series is a sequence of observations on a variable of interest. The variable is observed at discrete time points, usually equally spaced. Time series analysis involves describing the process or phenomena that generate the sequence. To forecast time series, it is necessary to represent the behavior of the process by a mathematical model that can be extended into the future.

It is required that the model be a good representation of the observations in any local segment of time close to the present. Once a valid model for the time series process has been established, an appropriate forecasting technique can be developed. Many of the models used to represent time series are algebraic or transcendental functions of time, or some composite model that combines both algebraic and transcendental components.

The idea of using a mathematical model to describe the behavior of a physical phenomenon is well established. In particular, it is sometimes possible to derive a model based on physical laws, which are capable of calculating the value of some timedependant variable, such a s quality, nearly exactly at any instant of time. Probably no phenomenon is deterministic because of the presence of unknown factors. In many problems it is required to consider a time-dependant phenomenon in which there are many unknown factors and for which it is not possible to write a deterministic model that 
allows exact calculation of the future behavior of the phenomenon. Nevertheless, it may be possible to derive a model that can be used to calculate the probability of a future value. The models for time series that are needed are stochastic models.

In a given lot of any commodity the number of parts that lie outside the mentioned specification limits is a very vital information as far as the work in process quality is concerned. This information which is usually specified in terms of percent defective i.e. the percentage of parts lying outside the specification limits is calculated by figuring out the dispersion of the specification limits from the process average. One can mention numerous factors like the workers capability, the machine condition etc, which are very obvious and are bound to affect the percent defective. But we can state some of the very fundamental factors, which will definitely control the value of percent defective in a lot.

Primarily the discipline of acceptance sampling relies on different distributions for its calculations. The value of percent defective for a lot size certainly depends upon the probability distributions, which are being followed. It is very well known that a process can be said to be composed of different stages. These stages can be primarily stratified into three stages viz. the infant or the early stage, the matured or the stable stage and finally the wear out or the decline stage. The percent defective during these stages of the process life differ a lot like for example one will agree that the percent defectives during the decline stage will be more than during the stable stage.

Process capability can be said to be the measure of the spread of the process. By this study, it becomes possible to know the percentage of the products, which will be produced within specified limits on either side of the process mean. Thus it also 
influences the value of percent defective to a certain extent. For example higher the process capability lower will be the value of percent defective for that lot.

\subsection{MODEL SELECTION}

In regression analysis the dependent variables $Y_{\mathrm{i}}$ are assumed to be independently distributed, but in reality they are not. They are correlated and follow a certain distribution. In the proposed model the dependent variable (Yi) is the percent defective which depends on the factors as explained above. The basic aim is to develop a regression model, which will explain the relationship between the dependent variable and the various independent variables. Since there exists a correlation between $Y_{I}$ 's a separate model needs to be developed to cater this aspect of the problem. This is being accomplished by developing a stochastic model. Some of the models considered are autoregressive model, moving average model, and ARIMA. Autoregressive model is a stochastic model, which can be extremely useful in representation of certain practically occurring series. To achieve greater flexibility in fitting of actual time series, it is sometimes advantageous to include both autoregressive and moving average terms in the model. ARIMA provides a powerful model for describing stationary and nonstationary time series. These models will be viewed as univariate time series models or for more than one time series. It is possible to build a model that uses the information content in other models to help forecast the dependent variable. These models are called transfer function models. The ARIMA model postulates that time series can be successfully represented as an output from a dynamic system to which the input is white noise and for which the transfer function can be parsimoniously expressed as the ratio of two 
polynomials. The data analysis tool employed for the identification of transfer function model is the cross correlation function between the input and the output. Multivariate transfer function models are used to relate several mutually interacting output time series to one or more time series.

With suitable inputs and outputs, a dynamic system can represent any process. The value $\mathrm{Y}_{\infty}(\mathrm{X})$ at which the discrete output from a stable system eventually comes to equilibrium when the input is held at a fixed level $\mathrm{X}$ is the steady state level of the output. Very often, over the range of interest, the relationship between $Y_{\infty}(X)$ and $X$ will be approximately linear. Hence if we use $\mathrm{Y}$ and $\mathrm{X}$ to denote deviations from convenient origins situated on the line, we can write the steady state relationship as

$$
\mathrm{Y}_{\infty}=\mathrm{gX}
$$

where $\mathrm{g}$ is the steady state gain, and it is understood that $\mathrm{Y}_{\infty}$ is a function of $\mathrm{X}$.

Corresponding to the continuous representation, discrete dynamic systems are often parsimoniously represented by the general linear difference equation also referred to as a transfer function model.

$$
\begin{gathered}
\left(1+\xi_{1} \nabla+\ldots \ldots \ldots \ldots . .+\xi_{r} \nabla^{r}\right) Y_{t}=g\left(1+\eta_{1} \nabla+\ldots \ldots \ldots \ldots . .+\eta_{s} \nabla^{s}\right) X_{t-b} \\
\left(1-\partial_{1} B-\ldots \ldots-\partial_{r} B^{r}\right) Y^{t}=\left(\omega_{0}-\omega_{1} B-\ldots \ldots \ldots \omega_{s} B^{s}\right) X_{t-b}
\end{gathered}
$$

In terms of backward shift operator $\mathrm{B}=1-\nabla$, the above equation may also be written as or as

$$
\partial(B) Y_{t}=\omega(B) X_{t-b}
$$


In these models $X_{t}$ and $Y_{t}$ were the deviations from equilibrium of the system input and output. In practice the system will be infected by disturbances, or noise, whose net effect it to corrupt the output predicted by the transfer function model by an amount $\mathrm{N}_{t}$. The combined transfer function-noise model may then be written as

$$
Y_{t}=\partial^{-1}(B) \omega(B) X_{t-b}
$$

The basic aim of this research is to develop a regression model in which the dependant variable is the percent defective and independent variables are as mentioned before. To incorporate the correlation between the independent variables, i.e., to predict percent defective from a set of previously recorded percent defective values, a time series model is being developed. From the above models a third model, which will cover the relationship of these two models, will be developed. This is being done using transfer function model.

In developing the final model, noise factor will be considered which will also take into account the disturbances in the correlation as well as the regression model.

\subsection{NEED FOR RESEARCH}

Most of the traditional forecasting approaches deal with inventory management, production planning, financial planning, staff scheduling, facilities planning or process control. In today's world where process and product quality is of utmost importance, it is necessary to forecast process quality as well so as to take corrective measures and also 
improve upon the existing process conditions. An approximate model can be developed using historical data to forecast work in process quality to be used in various applications.

In this research a similar model is being developed to forecast work in process quality. The variable of interest considered here is the percent non-conforming. This is assumed to follow beta distribution, which is a very general distribution. Not much research has been done in the past considering a variable percentage defective. This assumption is far more realistic as opposed to assuming a constant value of percent defective.

Forecasting using time series analysis and multiple regression is one of the most widely used approaches. Unfortunately very less research has been done in the field of forecasting work in process quality that lays out conditions under which effectiveness of a process can be studied. The objective of this research is to develop a model to forecast work in process quality and take corrective measures so as to improve over the existing conditions under the assumption of percent defective, which is the variable of interest being a random variable.

\subsection{RESEARCH OBJECTIVES}

The objective of this thesis is to develop a forecasting model using Holt-Winters method. This research will be aimed to present a procedure for interpolating, smoothing, and predicting work in process quality in seasonal time series with missing observations. The approach suggested by Wright (1986) for the Holt's method with nonseasonal data 
published at irregular time intervals will be extended to the Holt-Winters method in the seasonal case. The variable considered will be percent defective and the data will be generated using various processes like Autoregressive process and Autoregressive Moving Average process. The exponential smoothing approaches, including the Holt's and Holt-Winters methods, constitute the basic equipment of practical time series analysis. Wright has suggested such extensions for simple exponential smoothing and for Holt's method. This research extends this approach to the Holt-Winters method for seasonal method for seasonal data with missing observations.

\subsection{ORGANIZATION}

The organization of this thesis consists of five chapters. Chapter 2 comprises of the literature regarding the basic forecasting concepts and various approaches to forecasting. Time series analysis and exponential smoothing approaches are presented n this chapter. Chapter 3 presents the methodology followed to develop the model and lists the various steps involved. Chapter 4 consists of the results obtained using the methodology. Chapter 5 summarizes the conclusions drawn from this research and provides recommendations for future research. 


\section{LITERATURE SURVEY}

One of the operators which are employed extensively for stochastic models for forecasting and control is the backward shift operator $B$ which is defined by $B z_{t}=z_{t-1}$; hence $B^{m} z_{t}=z_{t-m}$. The inverse operation is the reverse of the forward shift operator $F=B^{-1}$ given by $F z_{t}=z_{t+1}$. Another important operator is the backward difference operator $\nabla$, which can be written in terms of $\mathrm{B}$, since

$\nabla z_{t}=z_{t}-z_{t-1}=(1-B) z_{t}$

In turn, $\nabla$ has for its inverse the summation operator $\mathrm{S}$ given by

$\nabla^{-1} z_{t}=S z_{t}=\sum_{j=0}^{\infty} z_{t-j}=(1-B)^{-1} z_{t}$

\subsection{LINEAR FILTER MODEL}

The stochastic models that are employed are based on the idea that a time series in which successive values are highly dependent can be usefully regarded as having been generated from a series of independent "shocks" $a_{t}$. These shocks are random drawings

from a fixed distribution usually assumed normal and having mean zero and variance $\sigma_{\mathrm{a}}^{2}$. Such a sequence of random variables is called a white noise process. The white noise process is supposed to be transformed to the process $z_{t}$ by what is called a linear filter as shown in figure 2.1. The linear filtering operation takes a weighted sum of previous observations so that

$z_{t}=\mu+a_{t}+\psi_{1} a_{t-1}+\psi_{2} a_{t-2}+\ldots .=\mu+\psi(B) a_{t}$ 
In general, $\mu$ is a parameter that determines the "level" of the process and

$\psi(B)=1+\psi_{1} B+\psi_{2} B^{2}+\ldots \ldots$

is the linear operator that transforms $\mathrm{a}_{\mathrm{t}}$ into $\mathrm{z}_{\mathrm{t}}$ and is called the transfer function of the filter.

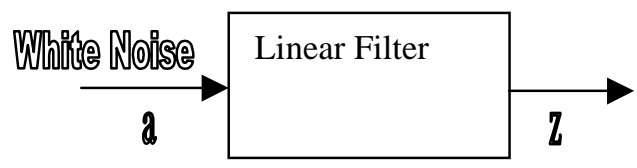

Figure 2.1

The sequence $\Psi_{i}$ formed by the weights may, theoretically, be finite or infinite. If this sequence is finite, or infinite and convergent, the filter is said to be stable and the process to be stationary. The parameter $\mu$ is then the mean about which the process varies. Otherwise, $\mathrm{z}_{\mathrm{t}}$ is non-stationery and $\mu$ has no specific meaning except as a reference point for the level of the process.

\subsubsection{AUTOREGRESSIVE PROCESS}

A stochastic model, which can be extremely useful in the representation of certain practically occurring series, is the so-called autoregressive model. In this model, the current value of the process is expressed as a finite, linear aggregate of previous values of the process and a shock. If the values of a process at equally spaced times $t, t-1, \ldots$ are $z_{t}$,

$$
\begin{aligned}
& \quad \tilde{z}=\phi_{1} \tilde{z}_{t-i}+\phi_{2} \tilde{z}_{t-2}+\ldots \ldots \ldots \ldots \ldots+\phi_{p} \tilde{z}_{t-p}+a_{t} \\
& \mathrm{z}_{\mathrm{t}-1} \ldots \text { then, }
\end{aligned}
$$

Where

$\tilde{z}_{t}=z_{t}-\mu \quad$ is the deviation from the mean 
is called an autoregressive process of order $\mathrm{p}$. The reason for this name is that a linear model relating a dependent variable $\mathrm{z}$ to a set of independent variables plus an error term is a regression model and $\mathrm{z}$ is said to be regressed on the independent variables. In equation (2.5) the variable $\mathrm{z}$ is regressed on the previous values of itself; hence the model is autoregressive. An autoregressive operator of order $\mathrm{p}$ can be defined by

$\Phi(B)=1-\Phi_{1} B-\Phi_{2} B^{2}-\ldots . . \Phi_{p} B^{p}$

and the autoregressive model may be written economically as

$\Phi(B) \tilde{z}_{t}=a_{t}$

The model contains $p+2$ unknown parameters $\mu, \phi_{1} \ldots \phi_{p}, \sigma_{a}^{2}$ which in practice have to be estimated from the data. The autoregressive model is a special case of the linear filter model. This can be seen by eliminating $\mathrm{z}_{\mathrm{t}-1}$ from the right hand side of equation (2.5) by substituting

$\tilde{z}_{t-1}=\Phi_{1} \tilde{z}_{t-2}+\Phi_{2} \tilde{z}_{t-3}+\ldots .+\Phi_{p} \tilde{z}_{t-p-1}+a_{t-1}$

Similarly, further substitution eventually yields an infinite series in the a's. Symbolically it can be written as

$\Phi(B) \tilde{z}_{t}=a_{t} \quad$ or $\quad \tilde{z}_{t}=\psi(\mathrm{B}) \mathrm{a}_{\mathrm{t}} \quad$ with $\quad \psi(B)=\Phi^{-1}(\mathrm{~B})$

Autoregressive process can be stationary or non-stationery. For the process to be stationary, the $\phi$ 's must be chosen such that they are the respective weights form a convergent series. 


\subsection{MOVING AVERAGE MODELS}

The autoregressive model expresses the deviation of the process as a finite weighted sum of p previous deviations of the process plus a random shock. Another kind of model, of great practical importance in the representation of observed time series, is the finite moving average process. Here $\tilde{z_{t}}$ is made linearly dependent on a finite number, q of previous a's. Thus,

$$
\tilde{z}_{t}=a_{t}-\theta_{1} a_{t-1}-\theta_{2} a_{t-2}-\ldots \ldots \ldots . .-\theta_{q} a_{t-q}
$$

is called a moving average (MA) process of order q. The name "moving average" is somewhat misleading because the weights which multiply the a's need not total unity nor need they be positive. If a moving average operator of order $\mathrm{q}$ is defined by

$\theta(B)=1-\theta_{1} B-\theta_{2} B^{2}-\ldots . .-\theta_{q} B^{q}$

then the moving average model may be written as

$\tilde{z}_{t}=\theta(B) a$

it contains $\mathrm{q}+2$ unknown parameters $\mu, \phi_{1}, \ldots \ldots, \phi_{\mathrm{q}}, \sigma_{\mathrm{a}}^{2}$ which in practice can be estimated from the data.

\subsubsection{MIXED AUTOREGRESSIVE-MOVING AVERAGE MODELS}

To achieve greater flexibility in fitting of actual time series, it is sometimes advantageous to include both autoregressive and moving average terms in the model. This leads to the mixed autoregressive-moving average model 
$\tilde{z}_{t}=\Phi_{1} \tilde{z}_{t-1}+\ldots . .+\Phi_{p} \tilde{z}_{t-p}+a_{t}-\theta_{1} a_{t-1}-\ldots . . \theta_{q} a_{t-q}$

which is equivalent to

$\Phi(B) \tilde{z}_{t}=\theta(B) a_{t}$

which employs $\mathrm{p}+\mathrm{q}+2$ unknown parameters $\mu ; \phi_{1} \ldots \phi_{\mathrm{p}} ; \theta_{1} \ldots \theta_{\mathrm{q}} ; \sigma_{\mathrm{a}}^{2}$ that are estimated from the data.

In practice, it is frequently true that adequate representation of actually occurring stationery time series can be obtained with autoregressive, moving average, or mixed models, in which $\mathrm{p}$ and $\mathrm{q}$ are not greater than 2 and often less than 2.

\subsubsection{NONSTATIONARY MODELS}

Many series encountered in practice exhibit nonstationary behavior and in particular do not vary about a fixed mean. Such series may nevertheless exhibit homogeneous behavior. In particular, although the general level about which fluctuations are occurring may be different at different times, the broad behavior of the series, when differences in level are allowed for, may be similar. Such behavior may be represented by a generalized autoregressive operator $\Phi(B)$, in which one or more of the zeroes of the polynomial $\Phi(B)$ (i.e. one or more of the roots of the equation $\Phi(B)=0$ ) is unity. Thus the operator $\Phi(\mathrm{B})$ can be written as $\Phi(\mathrm{B})=\Phi(\mathrm{B})(1-\mathrm{B})^{\mathrm{d}}$ where $\Phi(\mathrm{B})$ is a stationary operator. Thus, a general model that can represent homogeneous nonstationary behavior is of the form

$\Phi(B) z_{t}=\Phi(B)(1-B)^{d} z_{t}=\theta(B) a_{t}$

or $\Phi(B) w_{t}=\theta(B) a_{t}$

where $w_{t}=\nabla^{d} z_{t}$ 
Homogeneous nonstationery behavior can therefore be represented as a model which calls for the d'th difference of the process to be stationery. In practice $\mathrm{d}$ is usually 0,1 , or at most 2 .

The process defined by the above two equations provides a powerful model for describing stationary and nonstationary time series and is called an autoregressive integrated moving average (ARIMA) process, of order (p,d,q). The process is defined by $w_{t}=\phi_{1} w_{t-1}+\ldots \ldots .+\phi_{p} w_{t-p}+a_{t}-\theta_{1} a_{t-1}-\ldots \ldots . .-\theta_{q} a_{t-q} \quad$ where $\quad w_{t}=\nabla^{d} z_{t}$

If $w_{t}$ is replaced by $z_{t}-\mu$, when $d=0$, the above model will include the stationery mixed model as a special case and the pure autoregressive model and the pure moving average model. The relationship which is inverse to the relationship in equation (2.12a) is $\mathrm{z}_{\mathrm{t}}=\mathrm{S}^{\mathrm{d}} \mathrm{W}_{\mathrm{t}}$ where $\mathrm{S}$ is the summation operator and is defined by

$$
S w_{t}=\sum_{j=0}^{\infty} w_{t-j}=w_{t}+w_{t-1}+w_{t-2}+\ldots \ldots \ldots \ldots
$$

thus the ARIMA process can be generated from white noise by means of three filtering operations as indicated by the block diagram of figure 2.2. The first filter has input $a_{t}$, transfer function $\theta(B)$ and output $e_{t}$, where

$e_{t}=a_{t}-\theta_{1} a_{t-1}-\ldots . .-\theta_{q} a_{t-q}=\theta(B) a_{t}$

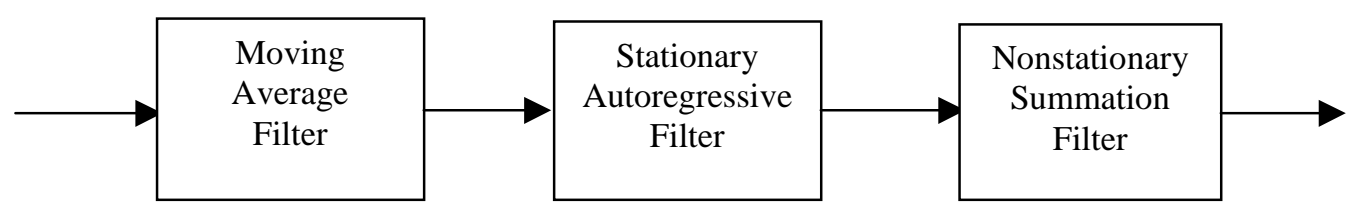

Figure 2.2 Block Diagram for ARIMA model 
The second filter in the above diagram has input $e_{t}$, transfer function $\phi^{-1}(B)$ and output $w_{t}$ according to $w_{t}=\phi_{1} w_{t-1}+\ldots \ldots \ldots+\phi_{p} w_{t-p}+e_{t}=\phi^{-1}(B) e_{t}$

Finally, the third filter has input $\mathrm{w}_{\mathrm{t}}$ and output $\mathrm{z}_{\mathrm{t}}$, and has transfer function $\mathrm{S}^{\mathrm{d}}$.

\subsection{TRANSFER FUNCTION MODELS}

An important type of dynamic relationship between a continuous input and a continuous output, is that in which the deviations of input $\mathrm{X}$ and of output $\mathrm{Y}$, from appropriate mean values, are related by a differential equation of the form

$$
\left(1+\Xi_{1} D+\ldots \ldots \ldots+\Xi_{R} D^{R}\right) Y(t)=\left(H_{0}+H_{1} D+\ldots \ldots \ldots+H_{S} D^{S}\right) X(t-\tau)
$$

Where $\mathrm{D}$ is the differential operator $\mathrm{d} / \mathrm{dt}$, the $\Xi$ 's and H's are unknown parameters and $\tau$ is a parameter which measures the dead-time or pure delay between input and output. In a similar way, for discrete data, the transfer between output $\mathrm{Y}$ and input $\mathrm{X}$, each measured at equispaced times can be represented by the difference equation

$$
\left(1+\xi_{1} \nabla+\ldots \ldots \ldots \ldots \ldots+\xi_{r} \nabla^{r}\right) Y_{t}=g\left(1+\eta_{1} \nabla+\ldots \ldots \ldots \ldots . .+\eta_{s} \nabla^{s}\right) X_{t-b}
$$

In which the differential operator $\mathrm{D}$ is replaced by the difference operator $\nabla$. An equation of the above form containing only a few parameters may often be used as an approximation to a dynamic relationship, whose true nature is more complex. This linear model can be written in terms of past values of input and output by substituting $B=1-\nabla$,

$$
\left(1-\partial_{1} B-\ldots \partial_{r} B^{r}\right) Y_{t}=\left(\omega_{0}-\omega_{1} B-\ldots \ldots-\omega_{s} B^{s}\right) X_{t-b}=\left(\omega_{0} B^{b}-\omega_{1} B^{b+1}-\ldots . \omega_{s} B^{b+s}\right) X
$$


or

$\partial(B) Y_{t}=\omega(B) B^{b} X_{t}=\Omega(B) X_{t}$

alternatively, it can also be said that the output $\mathrm{Y}_{\mathrm{t}}$ and the input $\mathrm{X}_{\mathrm{t}}$ are linked by a linear filter

$Y_{t}=v_{0} X_{t}+v_{1} X_{t-1}+v_{2} X_{t-2}+\ldots \ldots . .=v(B) X_{t}$

for which the transfer function

$v(B)=v_{0}+v_{1} B+v_{2} B^{2}+\ldots \ldots$

can be expressed as a ratio of two polynomials

$v(B)=\Omega(B) / \partial(B)=\partial^{-1}(B) \Omega(B)$

the linear filter is said to be stable if the above series converges for $|\mathrm{B}| \leq 1$. The series of weights, which appear in the transfer function, is called the impulse response function.

\subsubsection{PARSIMONY}

Mathematical models contain certain constants or parameters whose values must be estimated from the data. It is important, in practice, to employ the smallest number of parameters for adequate representation. The central role played by the principle of parsimony can be seen to be evident from the illustration that follows. Here a dynamic model of the following form can be considered [Box and Jenkins, 1976]

$Y_{t}=\left(\eta_{0}+\eta_{1} \nabla+\eta_{2} \nabla^{2}+\ldots \ldots \ldots+\eta_{s} \nabla^{s}\right) X_{t}$

Which can also be represented as 
$(1+\xi \nabla) Y_{t}=X_{t}$

The second model here contains only one parameter $\xi$ but, for s sufficiently large, could be represented approximately by the previous model. Because of experimental error, the relationship between the coefficients in the fitted equation may be misinterpreted. Thus, a relationship like the previous equation could be developed unnecessarily, which will contain s parameters. This could lead to poor estimation of the output $Y_{t}$ for given values of the input. It is necessary that the objective should be to obtain adequate but parsimonious models. Forecasting and control procedures could be seriously deficient if these models were either inadequate or unnecessarily prodigal in the use of parameters. Care and effort is needed in selecting the model. The process of selection is necessarily iterative; that is, it is a process of evolution, adaptation, or trial and error.

In practice many data series contain observations at irregular times whereas most forecasting methods are restricted to the case of equal time intervals between data points. The whole range of forecasting methods available in the literature from elementary smoothing methods to sophisticated Box Jenkins Analysis is almost entirely restricted in producing forecasts from data series with a fixed time interval between observations. Newbold (1981), in his reviews of the forecasting literature, deals entirely with such methods. In practice, however, data often occur intermittently.

Firstly, the frequency of reporting of a series may change. Equivalently older data may be aggregated to give a less frequent reporting interval when summary statistics are compiled. Secondly, the data may naturally occur at irregular time intervals. Thirdly, occasional data points may simply be unavailable in an otherwise regularly spaced series. 
This does not often occur in published time series. Interpolation can also be used when data points are missing but not in the first two cases described above.

\subsection{EXPONENTIAL SMOOTHING FOR IRREGULARLY SPACED DATA}

A time series $\mathrm{x}_{\mathrm{i}}$ occurring at regularly spaced times $i=0 . . . t$ can be "exponentially smoothed" by taking a weighted average of all the data points with a weighted factor (1 -

a) ${ }^{t-i}$ for $x_{i}, 0<\mathrm{a}<1$. A sequential method of calculating this weighted average is the standard equation for single exponential smoothing, Brown (1959):

$\bar{x}_{t}=(1-a) \bar{x}_{t-1}+a x_{t}$

The extension of (2.21) to the case of data at irregular times is obtained by extending the weighted average upon which (2.21) is based:

$\bar{X}_{n}=A_{n} V_{n}$,

Where the weighted average,

$A_{n}=\sum_{i=-\infty}^{n}(1-a)^{t_{n}-t_{i}} X_{i}$

And the normalizing factor,

$\frac{1}{V_{n}}=\sum_{i=-\infty}^{n}(1-a)^{t_{n}-t_{i}}$

The sequential form of (2.23) and (2.24) is: 
$A_{n}=b_{n} A_{n-1}+X_{n}$,

$\mathrm{V}_{\mathrm{n}}=V_{n-1} /\left(b_{n}+V_{n-1}\right)$,

where

$b_{n}=(1-a)^{t_{n}-t_{n-1}}$

Substituting (2.25) into (2.22) we obtain:

$\bar{X}_{n}=\left(1-V_{n}\right) \bar{X}_{n-1}+V_{n} X_{n}$

The extension of single exponential smoothing for data with irregular time spacing therefore consists of (2.26), (2.27) and (2.28) with initial conditions

$$
\begin{aligned}
& \bar{X}_{0}=X_{0} \\
& V_{0}=1-(1-a)^{q}
\end{aligned}
$$

The initial condition, (2.30), is an approximation obtained by assuming all data earlier than time $\mathrm{t}_{0}$ occurred at the same time spacing, $\mathrm{q}$, which is taken to be the average time spacing of the data. It is important to note that the extended form (2.28) and the regular form (1) correspond in the sense of following the basic principle new estimate $=(1-$ parameter $) *$ previous estimate + parameter $*$ new information.

In the extended form (2.28) the parameter changes reflecting changes in time spacing.

\subsection{HOLT'S METHOD FOR REGULARLY SPACED DATA}

This consists of fitting a trend line $l_{t}-m_{t}(t-i)$ to the data where the estimates of $l_{t}$ and $m_{t}$ are obtained sequentially from single exponential smoothing equations based on above relation. In the case of irregularly spaced data the following line can be fitted: 


$$
L_{n}-M_{n}\left(t_{n}-t_{i}\right)
$$

In the data $X_{I}$ using (2.31) as follows. At time, $t_{n}$ the previous estimate of $\mathrm{Ln}$ is $l_{n-1}+\left(t_{n}-t_{n-1}\right) M_{n-1} \quad$ and the new information is $\mathrm{X}_{\mathrm{n}}$.

The linearity of the approximation (2.32) implies that the slope does not change. Thus, the previous estimate of $L_{n}$ is $M_{n-1}$. The new information about $M n$ is $\left(L_{n}-L_{n}-1\right) /\left(t_{n}-t_{n}-1\right)$. This leads to the two equations:

$$
\begin{aligned}
& L_{n}=\left(1-V_{n}\right)\left[L_{n-1}+\left(t_{n}-t_{n-1}\right) M_{n-}\right]+V_{n} X_{n} \\
& M_{n}=\left(1-U_{n}\right) M_{n-1}+U_{n}\left(L_{n}-L_{n-1}\right) /\left(t_{n}-t_{n-1}\right)
\end{aligned}
$$

The derivations of this result follows ad hoc arguments rather than a strict mathematical proof, and this corresponds directly with the derivation given by Holt (1960). The parameter $\mathrm{V}_{\mathrm{n}}$ is updated by (2.26) and (2.27) and $\mathrm{U}_{\mathrm{n}}$ by the corresponding equations:

$$
U_{n}=U_{n-1} /\left(d_{n}+U_{n-1}\right)
$$

$$
d_{n}=(1-c)^{t_{n}-t_{n-1}}
$$

The extended Holt's method therefore maintains one of the primary advantages of the regular Holt's method, viz. the flexibility afforded by having two smoothing parameters: $0<\mathrm{a}<1, \quad 0<\mathrm{c}<1$.

Gilchrist (1976) recommends that the use of least squares regression on the first few data points as a means of obtaining initial conditions on the level and slope in Holt's method. We use the same approach with irregularly spaced data taking initial conditions for (2.32) and (2.33) from the slope B of the regression line and its intercept a at time $t_{0}$ : 
$L_{0}=A$

$M_{0}=B$

The initial condition on $\mathrm{U}_{\mathrm{n}}$ corresponds to (2.30)

$$
U_{0}=1-(1-c)^{q}
$$

The sequential nature of this forecasting procedure renders it highly efficient in terms of computer storage and time. The number of variables to be stored is 2 as compared to 5 for the regular Holt's method. The computation time is dependent on equations (2.27) and (2.35). If the time intervals between data points are integer multiples of a base value, then these equations can be evaluated by table look up. This is the case when the frequency of reporting changes, e.g. from quarterly to monthly, or when there are occasional observations missing in an otherwise regular series. If, however the data occur at completely irregular times then (2.27) and (2.35) must be evaluated by exponentiation, which is much more time consuming. The other variable contributor to computation time is $t_{n}-t_{n-1}$ in (2.32) and (2.33). In the case of changed reporting frequency or missing data in a regular series, $t_{n}-t_{n-1}$ can be scaled to be 1 for much of the analysis. This accelerates the computation of (2.32) and (2.33).

The literature on time series analysis with missing observations offers a broad range of methods that differ in their theoretical level, numerical complexity, and general applicability. From a practical point of view, extensions of the classical methods of exponential smoothing to the case of missing observations (or more generally, to the case of irregularly observed data) seem to be very important. The exponential smoothing approaches, including Holt's and Holt-Winters methods, constitute now the basic equipment of practical time series analysis. 
Wright (1986) has suggested such extensions for simple exponential smoothing and for Holt's method, which give good practical results in spite of their apparent numerical simplicity, as already discussed above. In this research this approach can be extended to the Holt-Winters method for seasonal data with missing observations. Although this extension, like Wright's, can be based on ad hoc arguments, it is expected to work well in practice. It can be treated in an acceptable way similar to seasonal time series even with $50 \%$ missing observations. Moreover, some theoretical investigations comparing the suggested procedure with the optimal results obtained by interpolating missing observations in the corresponding seasonal ARIMA models have been done.

\subsection{EXTENSION OF THE HOLT-WINTERS METHOD}

Let us consider observations $\mathrm{y}_{\mathrm{t} 1}, \mathrm{y}_{\mathrm{t} 2}, \ldots$ published at irregularly spaced times $\mathrm{t}_{1}<\mathrm{t}_{2}<$ $\ldots<\mathrm{t}_{\mathrm{n}}$ (in general these times need not be integers). In this case of the simple exponential smoothing and Holt's method for nonseasonal irregularly spaced data, as already discussed, Wright's (1986) argument consist of application of recursive scheme of the type:

Estimate for time $t_{n}=V_{t n} *$ new info at time $t_{n}+\left(1-V_{t n}\right) *$ estimate for time $t_{n}$ at time $t_{n-1}$

$$
V_{t_{n}}=\frac{V_{t_{n-1}}}{b_{t_{n}}+V_{t_{n-1}}}, \quad b_{t_{n}}=(1-\alpha)^{t_{n}-t_{n-1}}
$$

Where the smoothing coefficient $V_{\mathrm{tn}}$ is also calculated as

And the constant $\alpha$ would be used as the smoothing coefficient in the classical case of regularly observed data. For instance, in the case of simple exponential smoothing the formula (2.39) can be used as 
$\hat{y}_{t_{n}}=V_{t_{n}} y_{t_{n}}+\left(1-V_{t_{n}}\right) \hat{y}_{t_{n-1}}$

Where the R.H.S value is the smoothed value for time $t_{\mathrm{i}}$. The non-recursive form

$$
\begin{aligned}
& y_{t_{n}}=V_{t_{n}} \sum_{i=1}^{n}(1-\alpha)^{t_{n}-t_{i}} y_{t_{i}} \cdot \\
& V_{t_{n}}=1 / \sum_{i=1}^{n}(1-\alpha)^{t_{n}-t_{i}}
\end{aligned}
$$

In the formulae (2.40) and (2.41) shows that it is the weighted average of observations with decreasing exponential weights, reflecting the irregular character of observations. The schemes (2.39) and (2.40) can be used to extend the Holt-Winters method. Let $p$ denote the seasonal length. Further times $t_{1}<t_{2}<\ldots \ldots . t_{n}$ correspond to time points chosen from a regular seasonal pattern. It can also be assumed that for each of p seasonal periods at least one observation is present in an initial portion of data. If the observations are completely missing for a seasonal period, then it is impossible to estimate the seasonal index for this seasonal period.

Let the symbols $S_{t}, T_{t}$, and $I_{t}$, denote the estimated level, trend and seasonal index at time t, respectively. It is sufficient to give formulas of the extended Holt-Winters method for he additive case only since, then their form for the multiplicative case will be

$S_{t_{n}}=V_{t_{n}}\left(y_{t_{n}}-I_{t_{n}}\right)+\left(1-V_{t_{n}}\right)\left\lfloor S_{t_{n-1}}+\left(t_{n}-t_{n-1}\right) T_{t_{n-1}}\right\rfloor$

immediate.

$$
T_{t_{n}}=U_{t_{n}} \frac{S_{t_{n}}-S_{t_{n-1}}}{t_{n}-t_{n-1}}+\left(1-U_{t_{n}}\right) T_{t_{n-1}}
$$




$$
\begin{aligned}
& I_{t_{n}}=W_{t_{n}}\left(y_{t_{n}}-S_{t_{n}}\right)+\left(1-W_{t_{n}}\right) I_{t_{n}} \\
& V_{t_{n}}=\frac{V_{t_{n-1}}}{b_{t_{n}}+V_{t_{n-1}}}, \quad b_{t_{n}}=(1-\alpha)^{t_{n}-t_{n-1}} \\
& U_{t_{n}}=\frac{U_{t_{n-1}}}{d_{t_{n}}+V_{t_{n-1}}}, \quad d_{t_{n}}=(1-\gamma)^{t_{n}-t_{n-1}} \\
& W_{t_{n}}=\frac{W_{t_{n}}}{f_{t_{n}}+W_{t_{n}}}, \quad f_{t_{n}}=(1-\partial)^{\left(t_{n}-t_{n}^{\prime}\right) / p}
\end{aligned}
$$

Where $\mathrm{t}_{\mathrm{n}}{ }_{\mathrm{n}}$ denotes the largest value among $\mathrm{t}_{\mathrm{n}-1}, \mathrm{t}_{\mathrm{n}-2}, \ldots$. such that the time $\mathrm{t}_{\mathrm{n}}$ corresponds to the same seasonal period as time $t_{n}$. The constants $\alpha, \gamma, \delta(0<\alpha, \gamma, \delta<1)$ would be used as the smoothing coefficients by the classical Holt-Winters method in the case without missing observations.

The recursive formulas (2.43), (2.44), (2.46) and (2.47) for the level and trend correspond to the ones of the Holt's method extended by Wright (1986) and as already discussed earlier. The estimation of the seasonal indices according to (2.45), (2.47) can be explained as follows: the estimated seasonal index $\mathrm{I}_{\mathrm{tn}}$ combines the new observation adjusted by the estimated trend $\mathrm{S}_{\mathrm{tn}}$ with the most recent estimate $\mathrm{I}_{\mathrm{tn}}$ ' of the seasonal index for the same seasonal period.

The estimated level, trend, and seasonal indices enable us to calculate the

$$
\hat{y}_{t_{n+m}}\left(t_{n}\right)=S_{t_{n}}+m T_{t_{n}}+I_{\left(t_{n+m}\right)} \quad \text { for } \mathrm{m}=1,2, \ldots \ldots \ldots \ldots
$$

predicted values for $\mathrm{m}$ steps ahead. 
the smoothed values

$\hat{y}_{t_{n}}=S_{t_{n}}+I_{t_{n}}$

and the interpolated values

$\hat{y}_{t}=\hat{y}_{t}\left(t_{n-1}\right) \quad$ for $\mathrm{t}=\mathrm{t}_{\mathrm{n}-1}+1, \ldots \ldots \ldots, t_{n-1}$

where $\left(t_{n}+m\right)^{*}$ in equation 2.48 denotes the largest value among $t_{n}, t_{n-1}, \ldots$ such that the time $\left(t_{n}+m\right)^{*}$ corresponds to the same seasonal period as the time $t_{n}+m$, The initial values $\mathrm{S}_{0}, \mathrm{~T}_{0}, \mathrm{I}_{\mathrm{I}}, \mathrm{V}_{0}, \mathrm{U}_{0}, \mathrm{~W}_{\mathrm{I}}(\mathrm{I}=-\mathrm{p}+1,-\mathrm{p}+2, \ldots, 0)$, which are necessary to start the recursive formulas (2.46), (2.49) at time $\mathrm{t}_{0}=0$, can be calculated following the practical recommendation by Chatfield and Yar (1988) for the classical Holt-Winters method:

$$
\begin{aligned}
& T_{0}=\frac{\bar{y}_{k_{1}}-\bar{y}_{k_{0}}}{\left(k_{1}-k_{0}\right) p} \\
& S_{0}=\bar{y}_{k_{0}}-\left(k_{0} p-\frac{p-1}{2}\right) T_{0} \\
& I_{i}=\frac{1}{k(i)} \sum_{k} I_{i+k p}
\end{aligned}
$$

for $i=-p+1,-p+2, \ldots, 0$

$V_{o}=1-(1-\alpha)^{q}, \quad U_{0}=1-(1-\gamma)^{q}$

$W_{i}=1-(1-\partial)^{Q_{i}}$ for $i=-p+1,-p+2, \ldots, 0$,

where

$I_{i+k p}=y_{i+k p}-\left[\bar{y}_{k}+\left(i+\frac{p-1}{2}\right) T_{0}\right]$

and the following notation is used:

$k_{0}$ is such that the $k_{0}$ th season is the first nonempty season containing at least one observation; 
$k_{1}$ is minimal $\left(k_{1} \geq k_{0}+1\right)$ such that the first $k_{1}$ seasons contain at least one observation for each of the $p$ seasonal periods;

the sum in equation 2.52 is over all such $k$ that the observations $y_{i+k p}\left(k=1, \ldots, k_{1}\right)$ are present in the first $k_{1}$ seasons and $k(i)$ is the number of summands in this sum;

$q$ is the average time spacing of the data (i.e., every $q$ th observation is at our disposal on average)

$Q_{\mathrm{i}}$ is the average time spacing in the framework of the $i$ th seasonal period.

Since the assessment of the average time spacing $q$ at $Q_{\mathrm{i}}$ may be complicated, for the sake of simplicity it can be assumed that $q=Q_{-\mathrm{p}+1}=Q_{-\mathrm{p}+2}=\ldots=Q_{0}=1$ in the initial values. This assumption does not affect the forecasting model and seems to work well in practice.

\subsection{TRACKING SIGNAL TESTS}

Having developed statistics for estimating the mean and variance of forecast errors, the next step is to use these results to periodically monitor and control the forecasting process. Generally, a major concern is that the forecasts be unbiased, i.e., that the expected value of forecast error be zero. To test this hypothesis, the deviation of the estimate of the expected forecast error from zero can be measured, relative to the natural variability of this statistic about its true mean. If this deviation is large, the hypothesis would be rejected. 


\subsubsection{TESTS BASED ON THE CUMULATIVE FORECAST ERROR}

Suppose $\mathrm{Y}(\mathrm{T})$ is the sum of errors and this is used as the estimate of the expected error. At any time $\mathrm{T}, \mathrm{Y}(\mathrm{T})$ will undoubtedly differ from zero. There are two possible explanations: (1) The forecasts are unbiased and the deviation from zero is due to random variation; and (2) the forecasts are biased and the deviation is due to a combination of bias and random variation. To distinguish between the two cases, a measure of the natural random variation in the statistic $\mathrm{Y}(\mathrm{T})$ about its mean is needed. The standard deviation of $\mathrm{Y}(\mathrm{T}), \sigma_{\mathrm{y}}$, provides this information. Assuming that $\sigma_{\mathrm{y}}$ can be estimated, it can be concluded that the forecast is biased if $|\mathrm{Y}(\mathrm{T})|$ is greater than some multiple $\mathrm{K}_{1}$ of this estimate of the standard deviation, that is if

$$
\left|\frac{Y(T)}{\hat{\sigma}_{Y}}\right|>K_{1}
$$

To estimate $\sigma_{\mathrm{y}}$ a relationship between $\sigma_{\mathrm{y}}^{2}$ and $\sigma_{\varepsilon}^{2}$ is sought, in which the estimate of the latter can be used to obtain that of the former. Brown (refer), gives the following results for direct smoothing of constant and linear processes:

$$
\begin{array}{lrl}
\sigma_{Y}^{2}=\frac{1}{1-\beta^{2}} \sigma_{\varepsilon}^{2} & \text { constant } & 2.51 \\
\sigma_{Y}^{2}=\frac{2-2 \alpha^{2}+\alpha^{4}}{4-\alpha-4 \alpha^{2}+2 \alpha^{3}} \frac{1}{1-\beta^{2}} \sigma^{2}{ }_{\varepsilon} & \text { linear } & 2.52
\end{array}
$$

In general, for direct smoothing with an n-parameter forecasting model, brown found through simulation studies that the following relationship is approximately correct:

$$
\sigma_{Y}^{2}=\frac{1}{1-\beta^{2 n}} \sigma_{\varepsilon}^{2}
$$


Normally, the decision rule given by equation 2.50 is operated using the mean absolute deviation, $\Delta$ rather than $\sigma_{\mathrm{Y}}$. Equation 2.54 expresses the relation between estimate of the error variance and $\Delta$, so this can be substituted in equation 2.53 and then the result in equation 2.50 to obtain

$$
\begin{aligned}
& \hat{\sigma}_{Y} \approx\left[c_{1}\left(1-\beta^{2 n}\right)\right]^{-1 / 2}(1.25 \hat{\Delta}) \\
& \left|\frac{Y(T)}{\hat{\Delta}(T)}\right|>\frac{1.25 K_{1}}{\left[c_{1}\left(1-\beta^{2 n}\right)\right]^{1 / 2}} \equiv K_{2}
\end{aligned}
$$

The ratio $\mathrm{Y}(\mathrm{T}) / \Delta(\mathrm{T})$ is called the tracking signal, computed at time $\mathrm{T}$. Each period the absolute value of the tracking signal is compared with the constant $\mathrm{K}_{2}$ to test the hypothesis that the expected forecast error is zero. Since $K_{1}$ is chosen to be 2 or 3 , it turns out that $\mathrm{K}_{2}$ is typically between 4 and 6 .

\subsubsection{TESTS BASED ON THE SMOOTHED FORECAST ERROR}

It is also possible to develop a tracking signal test based on the smoothed error statistic $\mathrm{Q}(\mathrm{T})$. The hypothesis of an unbiased forecast would be rejected if,

$$
\left|\frac{Q(T)}{\hat{\Delta}(T)}\right|>K_{3}
$$

It is clear that the above ratio can never exceed 1.0. the proper value of $K_{3}$ is usually between 0.2 and 0.5 . For a constant model, where the level of time series is estimated by exponential smoothing, Brown has shown that

$$
\sigma_{Q}^{2}=\frac{\alpha}{(1+\beta)^{2}} \sigma_{\varepsilon}^{2}
$$

If the tracking signal test is of the form $\left|\mathrm{Q}(\mathrm{T}) / \sigma_{\mathrm{Q}}\right|>\mathrm{K}_{1}$, where $\mathrm{K}_{1}$ is usually between 2 and 5, substitution will yield 
$\left|\frac{Q(T)}{\hat{\sigma}_{e}}\right|>\frac{\sqrt{\alpha} K_{1}}{1+\beta}$

$\left|\frac{Q(T)}{\hat{\Delta}(T)}\right|>\frac{1.25 \sqrt{\alpha}}{1+\beta} K_{1} \equiv K_{3}$

Although the result in equation 2.57 is for a constant model, it is a good approximation for other models. From simulation runs using a seasonal model similar to equation 2.57 Brown found that $\sigma_{\mathrm{Q}} \approx \alpha^{0.5} \Delta$.

The smoothed error form of the tracking signal appears to have several advantages relative to the cumulative error version. Suppose that there was a large, but random, error in the forecast for a particular time period. This would increase both the smoothed error and the cumulative error, but assume that the increase is not quite enough to generate an out-of-control signal. Now suppose that several periods go by with small errors averaging zero. The cumulative error remains at its large value, but the smoothed error decreases toward zero. At some point of time, a second large, random error with the same sign as the previous one occurs. The cumulative error tracking signal would incorrectly generate an out-of-control signal, but the smoothed error version would not.

As a second example, suppose that the cumulative error tracking signal was just less than the control limit, and a period having zero forecast error occurs. The cumulative error would not change, but the mean absolute deviation estimate would become smaller. The net effect would be to increase the value of tracking signal, perhaps above the control limit. If the smoothed error tracking signal had been employed, the forecast would have caused the smoothed error to decrease along with the mean absolute deviation, resulting in little change in the tracking signal. 


\section{RESEARCH METHODOLOGY}

The literature on time series analysis with missing observations offers a broad range of methods that differ in their theoretical level, numerical complexity, and general applicability. The exponential smoothing approaches, including the Holt's and HoltWinters methods constitute the basic equipment of practical time series analysis. This research presents a procedure for interpolating, smoothing, and predicting in seasonal time series with missing observation. The approach suggested by Wright (1986) for the Holt's method for non-seasonal data published at irregular time intervals is extended to the Holt-Winters method in the seasonal case. Numerical examples demonstrate the procedure.

\subsection{ASSUMPTIONS}

The fraction non-conforming of a process has usually been assumed to be constant throughout the quality control literature. This is often not the case in the real world situations. In this research, the variation in fraction non-conforming is recognized and applied to the decision making criteria. The initial value in all the time series is generated using Beta distribution. The density function of the Beta distribution varies between 0 and 1 and the value of the fraction non-conforming varies between the same limits. Further values for fraction non-conforming are generated assuming the process to be Autoregressive or Moving Average or Autoregressive-Moving Average. This assumption is more realistic than the traditional approach. 
Another assumption used to generate the values of fraction non-conforming is that the error terms are normally distributed with mean zero and a certain variance. Three smoothing constants are used to develop the model and the values of these constants are assumed to be $\alpha=0.4, \gamma=0.1, \delta=0.4$, where $\alpha$ is the coefficient of level factor, $\gamma$ is the coefficient of trend factor and $\delta$ is the coefficient of seasonal factor in consideration as explained before. These values are "guestimates" of the smoothing coefficients as recommended by Chatfield and Yar, 1988. For performing the tracking signal analysis, values of $\alpha$ (smoothing constant) and $\beta$ are assumed to be between 0.1 and 0.3 and 0.7 and 0.95 respectively.

\subsection{SYMBOLS AND NOTATIONS}

The symbols and notations that were employed in the research are summarized below:

$\mathrm{y}_{\mathrm{t}} \quad$ Observation at time $\mathrm{t}$

$\mathrm{S}_{\mathrm{t}} \quad$ Level index at time $\mathrm{t}$

$\mathrm{T}_{\mathrm{t}} \quad$ Trend index ant time $\mathrm{t}$

$\mathrm{I}_{\mathrm{t}} \quad$ Seasonal index at time $\mathrm{t}$

$\alpha, \gamma, \delta \quad$ Smoothing constants, $0<\alpha, \gamma, \delta<1$

$\mathrm{V}_{\mathrm{t}} \quad$ Coefficient of level index at time $\mathrm{t}$

$\mathrm{U}_{\mathrm{t}} \quad$ Coefficient of trend index at time $\mathrm{t}$

$\mathrm{W}_{\mathrm{t}} \quad$ Coefficient of seasonal index at time $\mathrm{t}$

q $\quad$ Average time spacing

Q $\quad$ Average time spacing in the base seasons (assumed to be 1) 


\subsection{CALCULATION OF THE INITIAL VALUES}

The initial values of the level, trend, and seasonal indices $S_{0}, T_{0}$, and $I_{i}$ and the coefficients of these indices which are necessary to start the recursive formulae for calculation of the respective values at time $t=0$, are calculated following the practical recommendation by Chatfield and Yar (1988) for the classical Holt-Winters method. Since the assessment of the average time spacing, $q$, and $\mathrm{Q}_{\mathrm{i}}$ is complicated, for the sake of simplicity, it can be assumed that $\mathrm{q}=\mathrm{Q}_{\mathrm{p}+1}=\mathrm{Q}_{\mathrm{p}+2}=\ldots \ldots=\mathrm{Q}_{0}=1$ in the initial values of equation 3.5 (i.e., $\mathrm{V}_{0}=\alpha, \mathrm{U}_{0}=\gamma, \mathrm{W}_{-\mathrm{p}+1}=\mathrm{W}_{-\mathrm{p}+2}=\ldots \ldots=\mathrm{W}_{0}=\delta$ ). The formulae used are:

$T_{0}=\frac{\bar{y}_{k_{1}}-\bar{y}_{k_{0}}}{\left(k_{1}-k_{0}\right) p}$

$S_{0}=\bar{y}_{k_{0}}-\left(k_{0} p-\frac{p-1}{2}\right) T_{0}$

$I_{i}=\frac{1}{k(i)} \sum_{k} I_{i+k p}$

where,

$I_{i+k p}=y_{i+k p}\left[\bar{y}_{k}+\left(i+\frac{p-1}{2}\right)\right] T_{0}$

$V_{0}=1-(1-\alpha)^{q} ; \quad U_{0}=1-(1-\gamma)^{q}$

$W_{i}=1-(1-\delta)^{Q_{i}}$ for $i=-p+1,-p+2, \ldots, 0$.

and the following notation is used:

$k_{0}$ is such that the $k_{0}$ th season is the first non-empty season containing at least on observation; 
$k_{1}$ is minimal $\left(k_{1 \geq} k_{0}+1\right)$ such that the first $k_{1}$ seasons contain at least one observation for each of the $p$ seasonal periods;

the sum in equation 3.3 is over all such $k$ that the observation $y_{i+k p}\left(k=1, \ldots \ldots, k_{l}\right)$ are present in the first $k_{l}$ seasons and $\mathrm{k}(i)$ is the number of summands in this sum; $q$ is the average time spacing of the data.

\subsection{METHOD ADOPTED TO DEVELOP THE MODEL}

The model development started with generating the data. This was done by assuming the error terms to be normally distributed with mean zero and a range of values for variance. Further, the following steps were employed to generate the data and develop the final model:

\subsubsection{DATA GENERATION AND MODEL DEVELOPMENT}

1. The mean and variance of Beta distribution are functions of the parameters $\alpha$ and $\beta$. For a given set of mean and variance of Beta distribution, the values of the parameters $\alpha$ and $\beta$ were calculated using the following relations:

$$
\begin{aligned}
& \text { mean }=\alpha /(\alpha+\beta) \\
& \text { variance }=\alpha \beta /(\alpha+\beta)^{2}(\alpha+\beta+1)
\end{aligned}
$$

2. For a given combination of the parameters $\alpha$ and $\beta$, a random number was generated from the Beta distribution to represent the value of fraction non-conforming for the first period. Now, the data was assumed to be collected over four days with twelve observations each day and some of the observations missing. This initially required 48 data points. This was done by developing different processes like $\operatorname{AR}(1), \operatorname{AR}(2)$, 
$\operatorname{MA}(1), \operatorname{MA}(2), \operatorname{ARMA}(1,1), \operatorname{ARMA}(1,2), \operatorname{ARMA}(2,1)$, and $\operatorname{ARMA}(2,2)$. These processes were generated using the following relations:

$\mathrm{AR}(\mathrm{p})$ process : $y_{t}=\phi_{1} y_{t-1}+\phi_{2} y_{t-1}+\ldots \phi_{p} y_{t-p}+\varepsilon_{t}$

MA(q) process : $y_{t}=\varepsilon_{t}-\theta_{1} \varepsilon_{t-1}-\theta_{2} \varepsilon_{t-2}+\ldots \theta_{q} \varepsilon_{t-q}$

$\operatorname{ARMA}(\mathrm{p}, \mathrm{q})$ process : $y_{t}=\phi_{1} y_{t-1}+\phi_{2} y_{t-2}+\ldots+\phi_{p} y_{t-p}-\theta_{1} \varepsilon_{t-1}-\theta_{2} \varepsilon_{t-2}-\ldots \theta_{q} \varepsilon_{t-q}+\varepsilon_{t}$

where $\{\phi\}$ and $\{\theta\}$ are constants and different values are assumed for different models.

3. Another assumption here was of the number of missing observations. After the data was generated, different number of random numbers (integers) between 7 and 24 were generated. This was done so that some of the values could be assumed to be missing from the data. The minimum number of missing observations thus assumed was 7 and the maximum number was 24 . So after getting done with the first three steps, the required data for model development was available.

4. After all the data was generated, the next step now was to start the actual model development procedure. For this the first step was to calculate the initial values of the various indices and their respective coefficients. This was done using equations 3.1 through 3.5 .

5. The initial values thus obtained were then substituted in recursive equations 2.42 through 2.47 to get the level, trend and seasonal indices for all the periods. Values of smoothing coefficients were assumed to be $\alpha=0.4, \gamma=0.1$, and $\delta=0.4$ respectively for all the models.

6. On calculating all these values for the various indices, the smoothed values, the interpolated values and the forecasted values ( $\mathrm{m}$ periods ahead) could be calculated. This was done using equations 2.48 and 2.49. The predicted and the smoothed and 
interpolated values were then presented in the spreadsheet and graphical inspections were done for all the models, which indicated that the method gave practically acceptable results. This conclusion was also supported by the analysis of the corresponding residuals and further by doing the tracking signal analysis.

\subsubsection{TRACKING SIGNAL ANALYSIS}

1. As already discussed in the second chapter, to perform the tracking signal analysis, three variables are required. These variables are $\mathrm{Q}, \mathrm{Y}$ and $\Delta$. The assumption made during performing tracking signal analysis was that the model is correct and the initial parameter estimates are unbiased. So initial values of $Q(0)=0$ and $Y(0)=0$. Since there was some random variation assumed in the process so the initial value of $\Delta(0)$ has to be greater than 0 .

2. The first step was to calculate the errors, and this was the difference between the actual value and the predicted value for any time period. Next step was to initialize the parameters. As already discussed, $\mathrm{Q}(0)=0$ and $\mathrm{Y}(0)=0$. To calculate $\Delta(0)$, equation 2.55 was used. The value of $c_{1}$ can be obtained from Table 1 in Appendix 1, once a proper value of $\beta$ is assumed. Generally, value of $\beta$ is assumed to be between 0.75 and 0.95 .

3. After the initial values for $\mathrm{Q}, \mathrm{Y}$ and $\Delta$ were calculated, the next step was to calculate the same values for all the remaining periods. This was done using equations 2.56 through 2.58 .

4. Since the Holt-Winters method takes into consideration seasonal factor for forecasting, the ratio $\mathrm{Q}(\mathrm{T}) / \Delta(\mathrm{T})$ was then calculated and assuming $K_{l}=2$, the value 
of $K_{3}$ was calculated using equation 2.60. Examining the values, it could be concluded that the values remained well in control. This was expected, since the data for percent non-conforming were generated using random number generators.

\section{Example 1:}

In this example, a mean fraction non-conforming of 0.12 and a variance of 0.01 was considered. For simplicity sake and also to analyze all the models on the same basis, these values of mean and variance were considered for all the models developed.

1. Considering a mean of 0.12 and variance of 0.01 .

Therefore, $0.12=\alpha /(\alpha+\beta)$

and $0.01=\alpha \beta /(\alpha+\beta)^{2}(\alpha+\beta+1)$

Solving the two equations above, we get $\alpha=10.45$ and $\beta=76.6$

2. A random number generated from the Beta distribution using the given values of $\alpha$ and $\beta$ is 0.1504 , corresponding to a fraction non-conforming of $\mathrm{p}=15.04 \%$.

3. Error terms were generated using random number generator in Excel. This was done by generating 48 error terms corresponding the 48 periods into consideration. The mean of these error terms was assumed to be zero and variance was assumed to be 0.01 .

4. An $A R(1)$ process was then developed using the equation

$$
y_{t}=\phi_{1} y_{t-1}+\varepsilon_{t}
$$

Where, $y_{t}$ is the observation for the current period $t$,

$y_{t-1}$ is the observation for the previous period

$\varepsilon_{t}$ is the error term associated with the current period 
$\phi_{1}$ is the smoothing constant used for the AR(1) process and its value was assumed to be 0.65 .

5. After the data generation was complete, a random number between 7 and 24 was generated. This was done to eliminate some of the generated values for fraction nonconforming, since the model considers missing values. A random number generated thus was 16, which meant 12 values out of the 48 values generated were to be considered missing. The next step was to determine which values were to be eliminated. This was done again by generating 16 random numbers between 1 and 48 and the corresponding 12 values were eliminated. One assumption for generating 48 data points was that inspection was made 12 times a day, once every two hours, and data for four days was generated.

6. The above 5 steps gave the final data, which could now be used further for model development. The first step in model development was to determine the base seasons on which all the initial calculations could be performed. As already discussed, the first base season had to be chosen such that it would be the first non-empty season containing at least one observation. In this model, the first base season was season one (day one) and the second was season two (day two). Season two was the second base season, since the second base season had to be such that it contained observations for at least all those periods, which the first base season did not.

7. The averages of the values for the first and the second base seasons were then calculated and used for determining the initial values of the level and trend factors. The initial values for level and trend factors were calculated using equations 3.1 and 3.2 and these values were 0.91241 and -0.00181 respectively. Calculations for initial 
values of $S_{t}$ and $T_{t}$ are shown below and calculations for $I_{I}$ are shown in Table 2 of Appendix 1. Initial values for seasonal factor were calculated using equations 3.3 and 3.4 .

$$
\begin{aligned}
& T_{0}=\frac{(0.693 / 12)-(0.945 / 12)}{(2-1) 12}=-0.00181 \\
& S_{0}=0.057-(12-5.5) *(-0.00181)=0.09421
\end{aligned}
$$

8. After all the initializing was done, the next step was to calculate the values for trend, level and seasonal factors for all the periods. This was done using equations 2.42 through 2.47. For periods in which "data was not available" the factor $y_{t}$ was not considered and the negative sign after $y_{t}$ in equations 2.42 and 2.47 was removed. Sample calculations for period 3 in season one are shown below:

$$
\begin{aligned}
& S_{3}=V_{3}\left(y_{3}-I^{*}{ }_{3}\right)+\left(1-V_{3}\right)\left[S_{2}+\left(t_{3}-t_{2}\right) T_{2}\right]=0.4(0.015)+0.6[0.099+(1)(-0.00088)]=0.054 \\
& T_{3}=U_{3} \frac{S_{3}-S_{2}}{t_{3}-t_{2}}+\left(1-U_{3}\right) T_{2}=0.1 \frac{0.054-0.099}{3-2}+0.9(-0.00088)=-0.0052
\end{aligned}
$$

9. The sum of level and seasonal factors for a particular period gave the predicted value of fraction non-conforming for that period and $m$ period ahead forecast was obtained using equations 2.48 and 2.49. calculations for predicted as well as smothed and interpolated values for the third period in the first season are shown below: 
Predicted value for third period in first season :

$\hat{y}_{3}=S_{3}+I_{3}=0.054+0.0151=0.0691=6.91 \%$

Smoothed and Interpolated value for third period in first season (forecast made one period before)

$\hat{y}_{3}(2)=S_{3}+m T_{3}+I^{*}{ }_{3} \quad$ (where $\mathrm{m}$ is the time lag between the period for which forecast is done and the period at which forecast is made

$$
=0.054+1(-0.0052)+0.0151)=0.0638=6.38 \%
$$

10. The predicted values for $m=1, \ldots ., 12$ are given in the third column of Table 3.1 and Figure 3.1, while the smoothed and interpolated values are given in fourth column of Table 3.1 and in Figure 3.2. 


\begin{tabular}{|c|c|c|c|c|c|c|c|}
\hline Periods & 12 values missing & $Y^{\wedge}{ }_{t}\left(t_{1-1}\right)$ & $Y^{\wedge}{ }_{t}$ & Periods & 12 values missing & $\mathrm{Y}_{\mathrm{t}}\left(\mathrm{t}_{\mathrm{l}-1}\right)$ & $\mathrm{Y}_{\mathrm{t}}$ \\
\hline 1 & 0.1504 & 0.135395 & 0.135556 & 25 & & & 0.092282 \\
\hline 2 & 0.115854555 & 0.121499 & 0.122386 & 26 & 0.061867439 & 0.064584 & 0.06626 \\
\hline 3 & & & 0.069368 & 27 & 0.089388621 & 0.075749 & 0.075931 \\
\hline 4 & 0.111620009 & 0.095189 & 0.099016 & 28 & & & 0.094451 \\
\hline 5 & 0.109561231 & 0.098367 & 0.101273 & 29 & 0.069609018 & 0.071548 & 0.073217 \\
\hline 6 & 0.091609763 & 0.083507 & 0.085763 & 30 & 0.079455412 & 0.073443 & 0.07457 \\
\hline 7 & 0.080298013 & 0.07383 & 0.075559 & 31 & 0.072211875 & 0.064794 & 0.065134 \\
\hline 8 & 0.086905232 & 0.083329 & 0.084827 & 32 & 0.091401322 & 0.08337 & 0.082748 \\
\hline 9 & 0.082045314 & 0.078236 & 0.079446 & 33 & & & 0.058001 \\
\hline 10 & & & 0.07368 & 34 & 0.097444077 & 0.080191 & 0.080112 \\
\hline 11 & 0.06590498 & 0.056521 & 0.059726 & 35 & 0.097171636 & 0.087642 & 0.086362 \\
\hline 12 & 0.059514731 & 0.051604 & 0.054222 & 36 & 0.095165771 & 0.088506 & 0.086232 \\
\hline 13 & 0.067934571 & 0.069682 & 0.072845 & 37 & 0.090989548 & 0.097729 & 0.096194 \\
\hline 14 & & & 0.064092 & 38 & 0.104474331 & 0.10335 & 0.101483 \\
\hline 15 & 0.074920565 & 0.057862 & 0.059634 & 39 & 0.087662312 & 0.092757 & 0.091294 \\
\hline 16 & & & 0.085058 & 40 & & & 0.115443 \\
\hline 17 & & & 0.074216 & 41 & 0.089752895 & 0.089818 & 0.090152 \\
\hline 18 & 0.088947398 & 0.073664 & 0.074896 & 42 & 0.090064921 & 0.088967 & 0.089205 \\
\hline 19 & 0.071689058 & 0.063863 & 0.064271 & 43 & & & 0.063943 \\
\hline 20 & 0.08238714 & 0.077249 & 0.077066 & 44 & & & 0.054299 \\
\hline 21 & 0.069388444 & 0.068785 & 0.068503 & 45 & & & 0.028767 \\
\hline 22 & 0.08181357 & 0.080294 & 0.079714 & 46 & 0.110560216 & 0.082702 & 0.083841 \\
\hline 23 & 0.084202241 & 0.082507 & 0.081644 & 47 & 0.092698776 & 0.080792 & 0.080585 \\
\hline \multirow[t]{14}{*}{24} & 0.071793781 & 0.072923 & 0.072092 & 48 & 0.091477781 & 0.082054 & 0.080643 \\
\hline & & & & & & $\mathrm{Y}^{\wedge}{ }_{48+\mathrm{m}}(48)$ & \\
\hline & & & & 49 & & 0.089902 & \\
\hline & & & & 50 & & 0.091743 & \\
\hline & & & & 51 & & 0.085132 & \\
\hline & & & & 52 & & 0.109667 & \\
\hline & & & & 53 & & 0.103617 & \\
\hline & & & & 54 & & 0.103461 & \\
\hline & & & & 55 & & 0.088125 & \\
\hline & & & & 56 & & 0.097876 & \\
\hline & & & & 57 & & 0.08379 & \\
\hline & & & & 58 & & 0.12252 & \\
\hline & & & & 59 & & 0.108353 & \\
\hline & & & & 60 & & 0.097576 & \\
\hline
\end{tabular}

Table 3.1. Forecasting fraction defective for AR(1) process, 12 observations missing 




Figure 3.1 Generated vs. Predicted Values

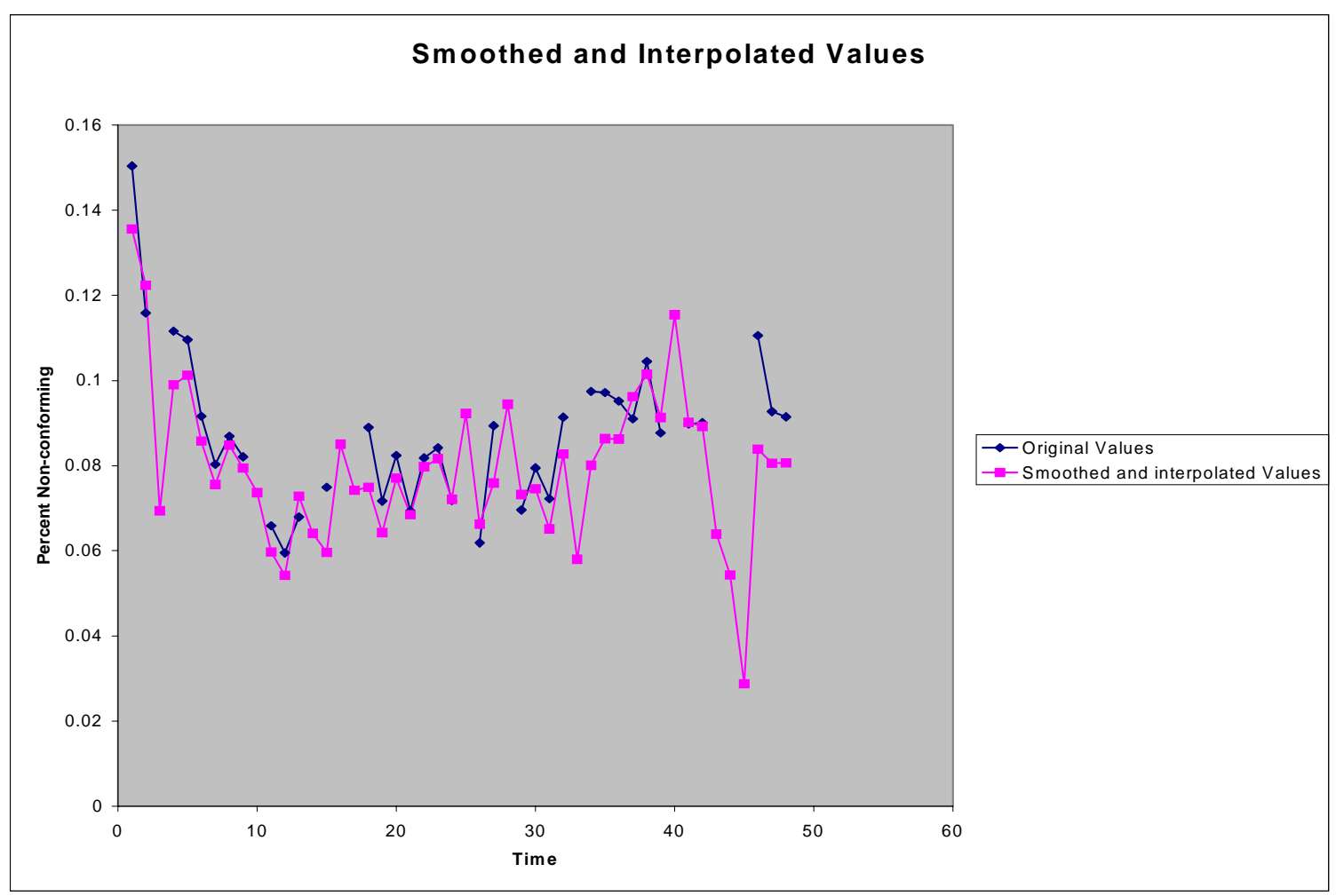

Figure 3.2. Generated vs. Forecasted values 


\begin{tabular}{|c|c|c|c|c|c|c|c|}
\hline Period & Errors & Error Sq. & Delta(t) & $\mathrm{Y}(\mathrm{T})$ & $\mathrm{Q}(\mathrm{T})$ & $\mathrm{Q}(\mathrm{T}) / \operatorname{Delta}(\mathrm{T})$ & $>0.42 ?$ \\
\hline 1 & 0.009505 & $9.03361 \mathrm{E}-05$ & 0.007866 & 0.009505 & 0.001045 & 0.13292022 & $n$ \\
\hline 2 & -0.01274 & 0.000162431 & 0.008354 & -0.00324 & -0.00033 & -0.03992753 & $n$ \\
\hline 4 & 0.008509 & $7.2402 \mathrm{E}-05$ & 0.008369 & 0.005269 & 0.000551 & 0.06580311 & $\mathrm{n}$ \\
\hline 5 & 0.003494 & 1.22048E-05 & 0.007882 & 0.008762 & 0.000845 & 0.10721196 & $n$ \\
\hline 6 & 0.000823 & 6.77208E-07 & 0.007176 & 0.009585 & 0.000843 & 0.11745072 & $n$ \\
\hline 7 & -0.00029 & 8.27853E-08 & 0.006487 & 0.009297 & 0.00073 & 0.11249428 & $\mathrm{n}$ \\
\hline 8 & -0.00262 & 6.83891E-06 & 0.0061 & 0.006682 & 0.000395 & 0.06479815 & $\mathrm{n}$ \\
\hline 9 & -0.00182 & 3.29789E-06 & 0.005671 & 0.004866 & 0.000174 & 0.03070236 & $n$ \\
\hline 11 & 0.004814 & 2.31764E-05 & 0.005586 & 0.00968 & 0.000638 & 0.11424526 & $\mathrm{n}$ \\
\hline 12 & 0.003809 & 1.45095E-05 & 0.005408 & 0.01349 & 0.000955 & 0.17663399 & $\mathrm{n}$ \\
\hline 13 & \begin{tabular}{|l|}
-0.00542 \\
\end{tabular} & 2.94116E-05 & 0.005409 & 0.008066 & 0.000317 & 0.05867137 & $n$ \\
\hline 15 & 0.014582 & 0.000212621 & 0.006327 & 0.022648 & 0.001744 & 0.27562531 & $\mathrm{n}$ \\
\hline 18 & -0.01458 & 0.000212701 & 0.007152 & 0.008064 & 0.000111 & 0.01551803 & $\mathrm{n}$ \\
\hline 19 & 0.006612 & 4.3714E-05 & 0.007098 & 0.014675 & 0.000761 & 0.10721588 & $n$ \\
\hline 20 & 0.003633 & 1.31985E-05 & 0.006752 & 0.018308 & 0.001048 & 0.15525411 & $\mathrm{n}$ \\
\hline 21 & -0.00104 & 1.07347E-06 & 0.00618 & 0.017272 & 0.00084 & 0.13588698 & $\mathrm{n}$ \\
\hline 22 & -0.00043 & 1.81475E-07 & 0.005605 & 0.016846 & 0.000713 & 0.1272536 & $\mathrm{n}$ \\
\hline 23 & 0.000365 & 1.33436E-07 & 0.005081 & 0.017211 & 0.000678 & 0.13352821 & $\mathrm{n}$ \\
\hline 24 & -0.00249 & 6.21254E-06 & 0.004822 & 0.014719 & 0.000361 & 0.07493651 & $\mathrm{n}$ \\
\hline 26 & -0.00351 & 1.23335E-05 & 0.004691 & 0.011207 & $-2.6 \mathrm{E}-05$ & -0.00553801 & $\mathrm{n}$ \\
\hline 27 & 0.012719 & 0.000161785 & 0.005494 & 0.023926 & 0.001249 & 0.22726553 & $\mathrm{n}$ \\
\hline 29 & -0.00202 & 4.07795E-06 & 0.005146 & 0.021907 & 0.000922 & 0.17910915 & $n$ \\
\hline 30 & 0.005559 & 3.09002E-05 & 0.005188 & 0.027466 & 0.001385 & 0.26707101 & $\mathrm{n}$ \\
\hline 31 & 0.006851 & 4.69374E-05 & 0.005354 & 0.034317 & 0.001932 & 0.360858 & $\mathrm{n}$ \\
\hline 32 & -0.00734 & 5.38041E-05 & 0.005552 & 0.026982 & 0.001005 & 0.18106945 & $\mathrm{n}$ \\
\hline 34 & 0.016842 & 0.000283645 & 0.006681 & 0.043824 & 0.002589 & 0.38750669 & $\mathrm{n}$ \\
\hline 35 & -0.00924 & 8.54104E-05 & 0.006937 & 0.034582 & 0.001406 & 0.20266102 & $\mathrm{n}$ \\
\hline 36 & 0.006157 & 3.79058E-05 & 0.006859 & 0.040739 & 0.001881 & 0.27423048 & $\mathrm{n}$ \\
\hline 37 & -0.00745 & 5.54608E-05 & 0.006918 & 0.033291 & 0.000948 & 0.13705855 & $n$ \\
\hline 38 & 0.000432 & 1.86717E-07 & 0.006269 & 0.033724 & 0.000897 & 0.14300627 & $n$ \\
\hline 39 & -0.00579 & 3.35138E-05 & 0.006221 & 0.027934 & 0.000228 & 0.03664629 & $\mathrm{n}$ \\
\hline 41 & $5.16 \mathrm{E}-05$ & 2.66463E-09 & 0.005604 & 0.027986 & 0.00021 & 0.0375336 & $\mathrm{n}$ \\
\hline 42 & 0.000967 & 9.35623E-07 & 0.005141 & 0.028953 & 0.000286 & 0.05564362 & $y$ \\
\hline 46 & 0.028431 & 0.000808303 & 0.00747 & 0.057384 & 0.003101 & 0.41508094 & $\mathrm{n}$ \\
\hline 47 & 0.012234 & 0.000149682 & 0.007946 & 0.069618 & 0.004014 & 0.50513964 & $y$ \\
\hline \multirow[t]{5}{*}{48} & 0.009372 & 8.78277E-05 & 0.008089 & 0.07899 & 0.00455 & 0.56247477 & $y$ \\
\hline & SSE & 0.002757916 & & & & & \\
\hline & SSE/34 & 8.11152E-05 & & & & & \\
\hline & K3 & 0.42 & & & & & \\
\hline & Delta(0) & 0.007683507 & & & & & \\
\hline
\end{tabular}

Table A.1.1 Tracking Signal Analysis \{ AR(1) process, 12 observations missing \} 


\subsubsection{Analysis of the developed models}

Various models were developed using the similar procedures and results were presented in similar form. The different models developed differed in their way of data generation, viz., data was generated using $\operatorname{AR}(1), \operatorname{AR}(2), \operatorname{MA}(1), \operatorname{MA}(2), \operatorname{ARMA}(1,1), \operatorname{ARMA}(1,2), \operatorname{ARMA}(2,1)$ and ARMA(2,2) and also in the number of "missing observations". The maximum number of missing observations considered was 23 (i.e., 47.9\%). The value of Root Mean Squared Forecast Error and graphical inspections of predicted as well as smoothed and interpolated values indicated that the method gave acceptable results. This conclusion is also supported by the analysis of tracking signal and also the corresponding residual time series. 


\section{ANALYSIS OF RESULTS}

The approach to forecasting work in process quality that was considered was HoltWinters method. The variable under consideration was fraction non-conforming and an assumption of some of the values being missing was made. The initial values of fraction nonconforming were generated for given values of mean and variance of Beta distribution and then adding the normally distributed error terms (with zero mean and given variance) to these values. Further values of fraction non-conforming were generated using various processes like Autoregressive, Autoregressive Moving Average process. Considering fraction non-conforming as the variable of interest, forecasting models using Holt-Winters method were developed and compared for effectiveness based on the errors and error variance obtained. The analysis of forecast errors was done using the method of tracking signals. The number of missing observations in each model was varied and the developed models gave acceptable results with as many as $40 \%$ of the observations missing. The results obtained for various processes with different number of missing observations were tabulated.

Almost all the models gave similar results irrespective of the process used or followed for data generation although some of the models gave somewhat varying results depending on the number of missing observations. It was seen in all the models that the coefficients of the trend and level factors remained constant throughout the forecasting process. This is attributed to the fact that these coefficients are smoothed based on the previous period in the same season. Further, it was observed that the estimated level factor adjusted based on level factor for the previous period as well as based on the most recent seasonal factor corresponding to the same seasonal period in which the observed percent non-conforming was not missing. It was also seen 
that the coefficient of seasonal factor combined the new observation adjusted by the estimated trend factor with the most recent estimate of the seasonal factor for the same seasonal period. The trend in all the models was observed to be constant but the seasonal index seemed to vary in a manner such that it was high during the first six periods and low during the last six periods of a season. Further, to avoid complexity, average time spacing in data was considered to be one period so the initial values of the coefficients of level and trend factors came out to be same as the smoothing constants used.

When the tracking signal exceeds the control limit for three or more successive periods, this seems to give a strong indication that something is wrong with the forecasting system. The action to be taken depends upon the likely cause of the out-of-control condition. The possible causes of variation that were observed were:

1. The out-of-control point is caused by random variation alone. In this case no action is to be taken.

2. The parameter estimates used in the forecasting model are not accurate because the true parameter values have changed over time. The model form is still correct, but improved estimates of the model parameters are required.

\subsection{RESULTS FOR AR (1) PROCESS}

The values of fraction non-conforming were generated using the Autoregressive "AR(1)" process in which values for a certain period are obtained by regressing over the values in the previous period. The estimated and forecasted values of percent non-conforming were obtained using the Holt-Winters method as described in chapter 2. The data for AR(1) process was generated by assuming a mean of 0.1 and the value of $\phi_{1}$ was assumed to be 0.65 . Data was 
generated for 48 periods and 12 values of the total values generated were assumed to be missing.

The results showed that unbiased forecasts were obtained for almost all the periods using the method and forecasts for 2 periods out of the total 48 were biased. It could also be seen that the level and trend of the forecasted values were similar to those of the generated data, which confirmed that the method gave acceptable results. Further, for periods where the forecast was biased, it could be seen that either data for the previous period was missing which caused the forecast for the following period to be biased or the period was preceded by a period where the forecast was biased. This bias in the forecast can be justified by the fact that the data was generated using "Autoregressive" process and hence if forecast for a particular period was biased then there was a high probability of the forecast for the following period being biased.

The seasonal index for any period is one of the important factors that decide the forecast and the estimated values for that particular period. Since the forecast and estimation for a period include the seasonal index as one of the major factors any variation in the seasonal index could affect these values and thus cause the forecast to be biased and go out of acceptable limits. So one major reason for the forecast being biased was that data for the same seasonal period in one of the preceding seasons was missing which caused variation in the value of the seasonal index of that period thus giving a biased forecast.

The results which show the generated values and the estimated as well as forecasted values for data generated using $\mathrm{AR}(1)$ process have been listed in table 4.1.1 and also in the following sections. The number of missing observations for each of the models is different and it can be seen that this method gives acceptable results with as many as $40 \%$ of the total observations missing. The results have been tabulated and graphs showing the generated data and the estimated as well as smoothed and interpolated values against time are shown for different 
number of observations missing. Analysis of tracking signal, which shows whether the forecast for a particular period is biased or unbiased is presented in Appendix 1. 


\subsection{RESULTS FOR AR(2) PROCESS}

The values of fraction non-conforming were generated using the AR(2) process in which values for a certain period are obtained by regressing over the values in the preceding two periods. The estimated and forecasted values of percent non-conforming were obtained using the Holt-Winters method as described in chapter 2. The data for $\operatorname{AR}(2)$ process was generated by assuming a mean of 0.1 and the values of $\phi_{1}$ and $\phi_{2}$ were assumed to be 0.45 and 0.54 respectively. Data was generated for 48 periods and 16 values of the total values generated were assumed to be missing. Results obtained by using this method of data generation were similar to those obtained for AR(1) process. The results have been tabulated and graphs showing the generated data and the estimated as well as smoothed and interpolated values against time are shown for different number of observations missing. Analysis of tracking signal, which shows whether the forecast for a particular period is biased or unbiased is presented in Appendix 1. 


\begin{tabular}{|c|c|c|c|c|c|c|c|}
\hline Period & 12 obs. Missing & Prediction & Forecast & Period & 12 obs. Missing & Prediction & Forecast \\
\hline 1 & 0.1504 & 0.135556 & 0.140895 & 31 & 0.072211875 & 0.065641 & 0.066304 \\
\hline 2 & 0.115854555 & 0.124366 & 0.129293 & 32 & 0.091401322 & 0.083369 & 0.084991 \\
\hline 3 & & 0.077024 & & 33 & & 0.058535 & \\
\hline 4 & 0.111620009 & 0.103123 & 0.104854 & 34 & 0.097444077 & 0.080384 & 0.081124 \\
\hline 5 & 0.109561231 & 0.105738 & 0.108067 & 35 & 0.097171636 & 0.086297 & 0.088319 \\
\hline 6 & 0.091609763 & 0.090327 & 0.093031 & 36 & 0.095165771 & 0.086457 & 0.089649 \\
\hline 7 & 0.080298013 & 0.080083 & 0.083081 & 37 & 0.090989548 & 0.09682 & 0.099504 \\
\hline 8 & 0.086905232 & 0.089244 & 0.092282 & 38 & 0.104474331 & 0.102413 & 0.105595 \\
\hline 9 & 0.082045314 & 0.083729 & 0.086884 & 39 & 0.087662312 & 0.092079 & 0.095089 \\
\hline 10 & & 0.083342 & & 40 & & 0.118391 & \\
\hline 11 & 0.06590498 & 0.06373 & 0.064333 & 41 & 0.089752895 & 0.090462 & 0.091721 \\
\hline 12 & 0.059514731 & 0.057995 & 0.058827 & 42 & 0.090064921 & 0.089829 & 0.09124 \\
\hline 13 & 0.067934571 & 0.076351 & 0.076331 & 43 & & 0.064676 & \\
\hline 14 & & 0.070109 & & 44 & & 0.052666 & \\
\hline 15 & 0.074920565 & 0.061775 & 0.062307 & 45 & & 0.026299 & \\
\hline 16 & & 0.087643 & & 46 & 0.110560216 & 0.083098 & 0.082279 \\
\hline 17 & & 0.074957 & & 47 & 0.092698776 & 0.079869 & 0.080393 \\
\hline 18 & 0.088947398 & 0.075031 & 0.075278 & 48 & 0.091477781 & 0.080254 & 0.082078 \\
\hline 19 & 0.071689058 & 0.064894 & 0.06592 & 49 & & & 0.090037 \\
\hline 20 & 0.08238714 & 0.077982 & 0.079601 & 50 & & & 0.092479 \\
\hline 21 & 0.069388444 & 0.069602 & 0.071359 & 51 & & & 0.085695 \\
\hline 22 & 0.08181357 & 0.081452 & 0.083436 & 52 & & & 0.111344 \\
\hline 23 & 0.084202241 & 0.082519 & 0.084889 & 53 & & & 0.105227 \\
\hline 24 & 0.071793781 & 0.073215 & 0.075663 & 54 & & & 0.104897 \\
\hline 25 & & 0.094615 & & 55 & & & 0.090009 \\
\hline 26 & 0.061867439 & 0.067072 & 0.066857 & 56 & & & 0.099973 \\
\hline 27 & 0.089388621 & 0.076684 & 0.077859 & 57 & & & 0.08668 \\
\hline 28 & & 0.096102 & & 58 & & & 0.127765 \\
\hline 29 & 0.069609018 & 0.073248 & 0.072738 & 59 & & & 0.112923 \\
\hline 30 & 0.079455412 & 0.074954 & 0.074894 & 60 & & & 0.102137 \\
\hline
\end{tabular}

Table 4.1.1 Forecasting fraction non-conforming for data generated using $\operatorname{AR}(1)$ process (12 observations missing) 


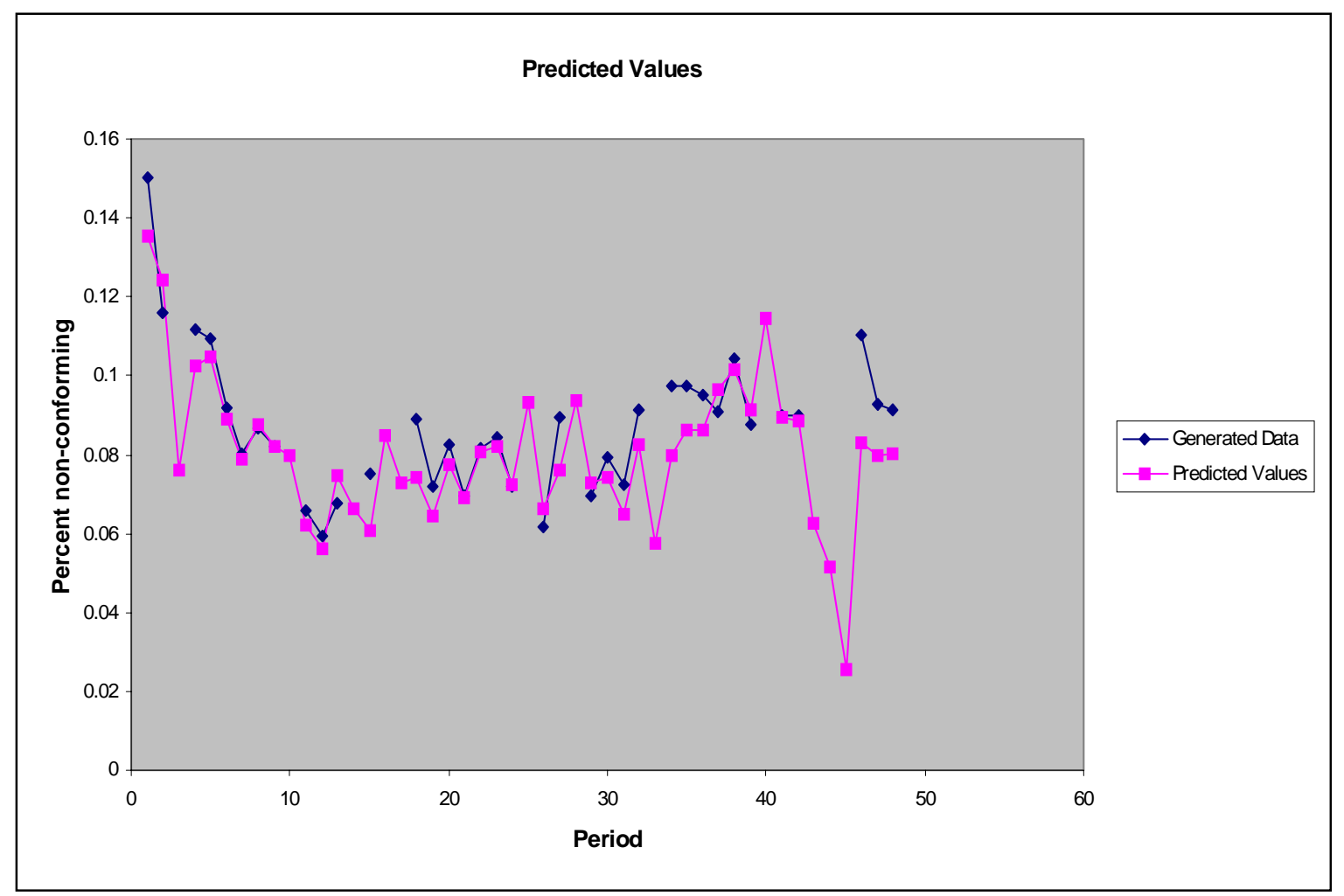

Figure 4.1.1(a) Generated vs. Predicted values, AR(1) process, 12 observations missing

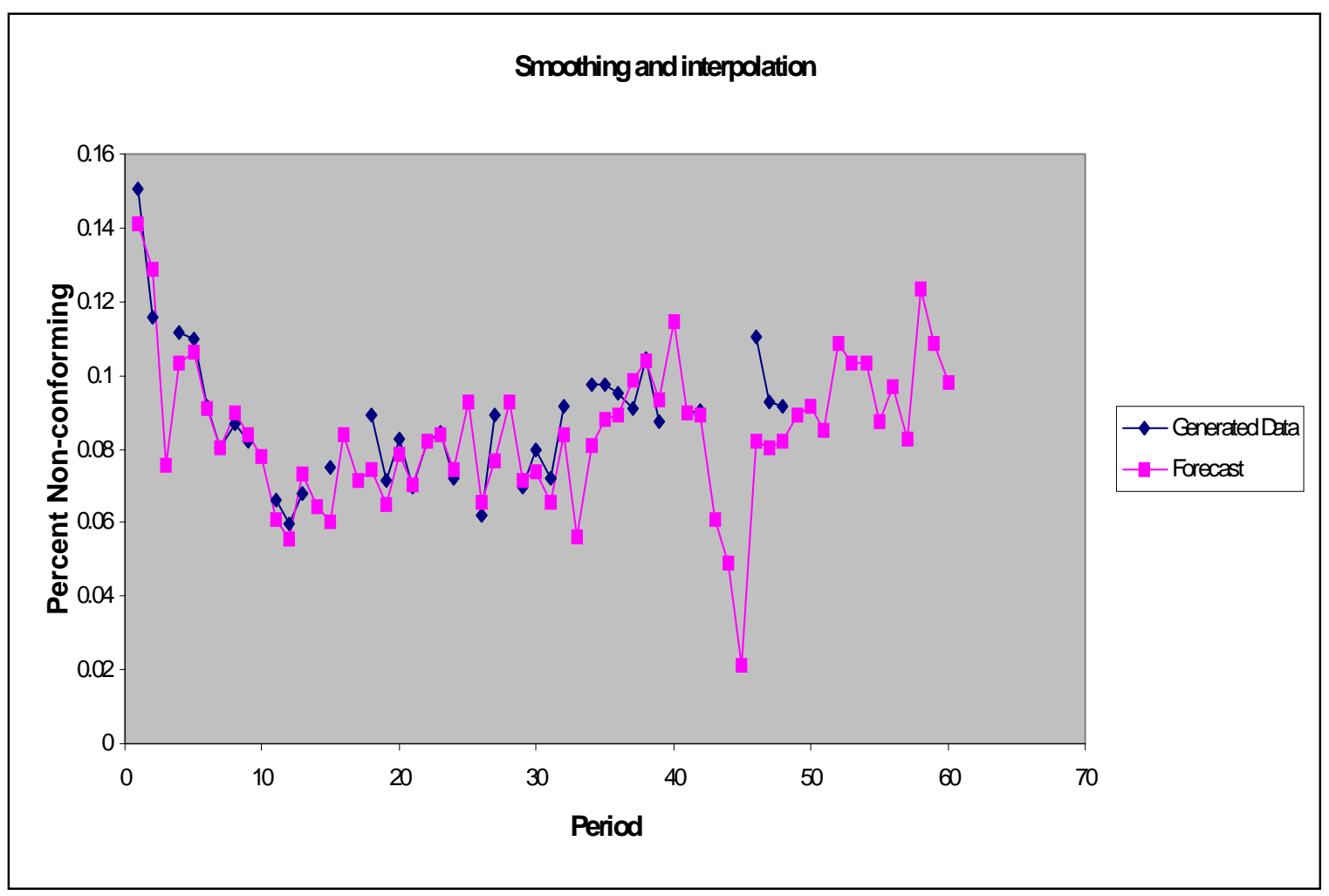

Figure 4.1.1(b) Generated vs. Forecasted values, AR(1) process, 12 observations missing 


\begin{tabular}{|c|c|c|c|c|c|c|c|}
\hline Periods & 16 values missing & Estimate & Forecast & Periods & 16 values missing & Estimate & Forecast \\
\hline 1 & 0.1504 & 0.135556 & 0.155395 & 31 & 0.07 & 0.061885 & 0.06173 \\
\hline 2 & 0.115854555 & 0.129586 & 0.147899 & 32 & 0.091401322 & 0.079206 & 0.080406 \\
\hline 3 & & 0.095576 & 0.108301 & 33 & & 0.050163 & 0.049018 \\
\hline 4 & 0.111620009 & 0.112218 & 0.124876 & 34 & 0.097444077 & 0.07904 & 0.07994 \\
\hline 5 & 0.109561231 & 0.115129 & 0.127169 & 35 & 0.097171636 & 0.086924 & 0.088962 \\
\hline 6 & 0.091609763 & 0.099457 & 0.110625 & 36 & 0.095165771 & 0.086877 & 0.089835 \\
\hline 7 & 0.080298013 & 0.090158 & 0.100231 & 37 & 0.090989548 & 0.094224 & 0.096724 \\
\hline 8 & 0.086905232 & 0.096286 & 0.105316 & 38 & 0.104474331 & 0.102473 & 0.105195 \\
\hline 9 & 0.082045314 & 0.090111 & 0.098245 & 39 & 0.087662312 & 0.091089 & 0.093431 \\
\hline 10 & & 0.09646 & 0.100827 & 40 & & 0.109488 & 0.109606 \\
\hline 11 & 0.06590498 & 0.065264 & 0.069702 & 41 & 0.089752895 & 0.087614 & 0.087969 \\
\hline 12 & 0.059514731 & 0.06359 & 0.067575 & 42 & 0.090064921 & 0.088746 & 0.089248 \\
\hline 13 & 0.067934571 & 0.080843 & 0.083394 & 43 & & 0.075103 & 0.073685 \\
\hline 14 & & 0.07452 & 0.07534 & 44 & & 0.062335 & 0.059978 \\
\hline 15 & 0.074920565 & 0.063658 & 0.066075 & 45 & & 0.030815 & 0.027154 \\
\hline 16 & & 0.085558 & 0.086494 & 46 & 0.110560216 & 0.085318 & 0.084462 \\
\hline 17 & & 0.069765 & 0.069632 & 47 & 0.092698776 & 0.082091 & 0.082413 \\
\hline 18 & 0.088947398 & 0.072847 & 0.074503 & 48 & & 0.056488 & 0.054922 \\
\hline 19 & & 0.044025 & 0.043123 & 49 & & & 0.05256 \\
\hline 20 & 0.08238714 & 0.065059 & 0.066081 & 50 & & & 0.054519 \\
\hline 21 & 0.069388444 & 0.061792 & 0.063658 & 51 & & & 0.042654 \\
\hline 22 & 0.08181357 & 0.079012 & 0.081276 & 52 & & & 0.057701 \\
\hline 23 & & 0.052534 & 0.051821 & 53 & & & 0.05345 \\
\hline 24 & 0.071793781 & 0.057601 & 0.058465 & 54 & & & 0.051196 \\
\hline 25 & & 0.071084 & 0.070565 & 55 & & & 0.043167 \\
\hline 26 & 0.061867439 & 0.061432 & 0.060975 & 56 & & & 0.045528 \\
\hline 27 & 0.089388621 & 0.072766 & 0.074156 & 57 & & & 0.023101 \\
\hline 28 & & 0.085693 & 0.085721 & 58 & & & 0.06201 \\
\hline 29 & 0.069609018 & 0.070095 & 0.070054 & 59 & & & 0.046286 \\
\hline 30 & & 0.057296 & 0.055994 & 60 & & & 0.037689 \\
\hline
\end{tabular}

Table 4.1.2 Forecasting fraction non-conforming for data generated using $\operatorname{AR}(1)$ process (16 observations missing) 


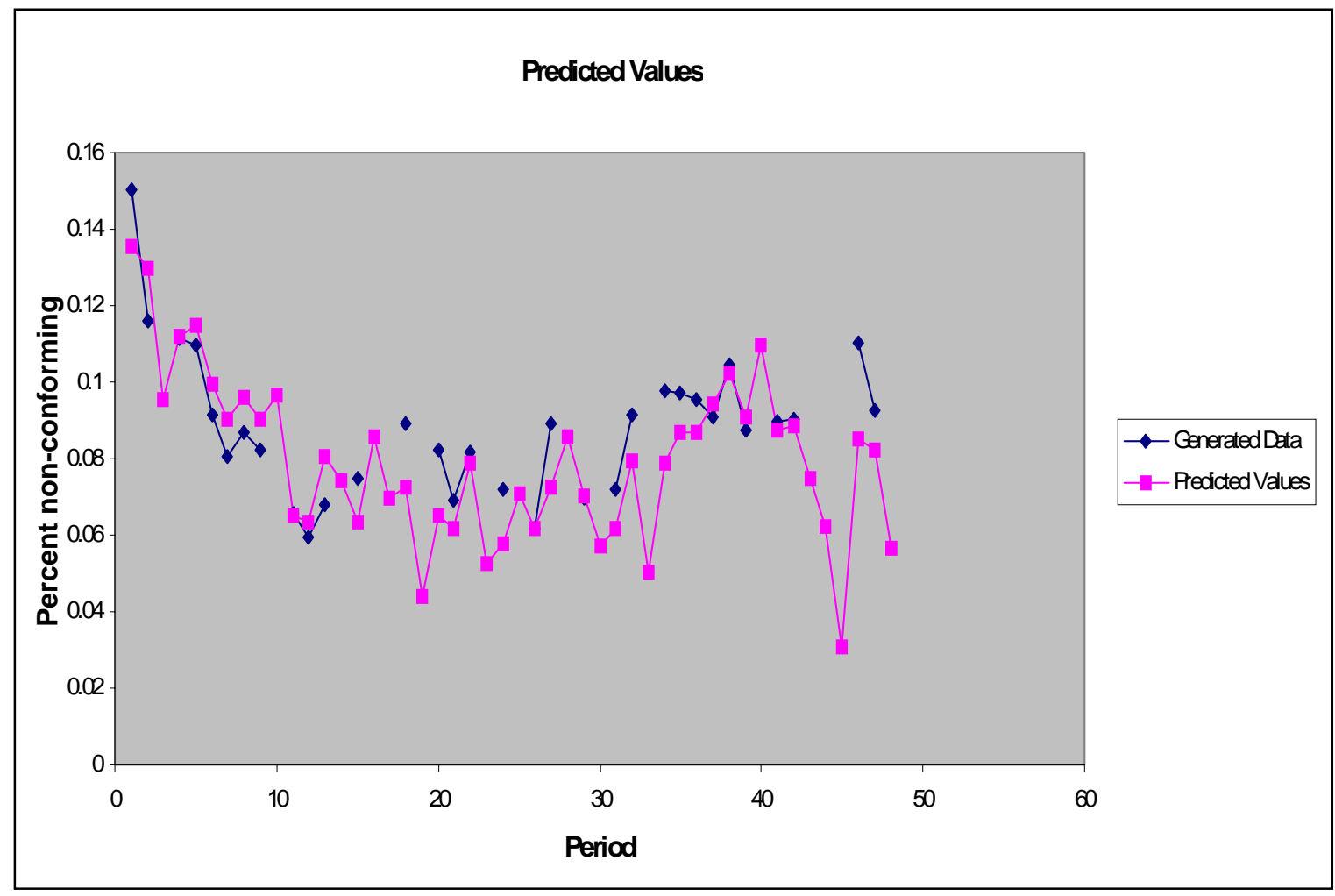

Figure 4.1.2(a) Generated vs. Predicted values, AR(1) process, 16 observations missing

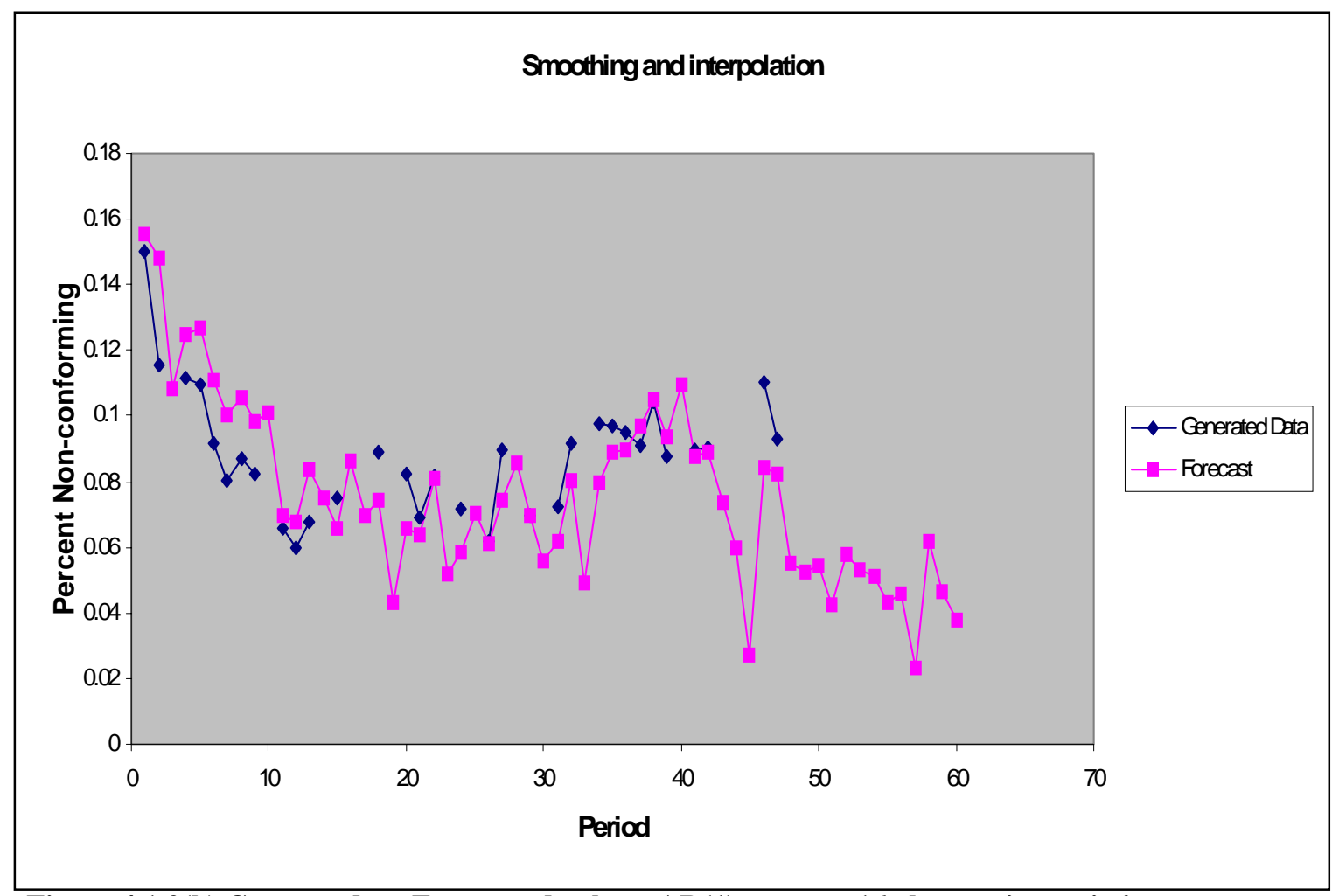

Figure 4.1.2(b) Generated vs. Forecasted values, AR(1) process, 16 observations missing 


\begin{tabular}{|c|c|c|c|c|c|c|c|}
\hline Period & 18 obs. Missing & Estimate & Forecast & Period & 18 obs. Missing & Estimate & Forecast \\
\hline 1 & & 0.050186 & 0.068939 & 31 & 0.072211875 & 0.065481 & 0.066833 \\
\hline 2 & 0.115854555 & 0.107284 & 0.126989 & 32 & & 0.059242 & 0.05838 \\
\hline 3 & & 0.065522 & 0.08107 & 33 & & -0.03038 & -0.03523 \\
\hline 4 & 0.111620009 & 0.105506 & 0.121733 & 34 & 0.097444077 & 0.069515 & 0.072771 \\
\hline 5 & 0.109561231 & 0.112386 & 0.128299 & 35 & 0.097171636 & 0.075077 & 0.080787 \\
\hline 6 & & 0.096299 & 0.108443 & 36 & & 0.043297 & 0.046462 \\
\hline 7 & 0.080298013 & 0.078276 & 0.090644 & 37 & 0.090989548 & 0.069834 & 0.076 \\
\hline 8 & 0.086905232 & 0.091532 & 0.103387 & 38 & 0.104474331 & 0.104505 & 0.110667 \\
\hline 9 & 0.082045314 & 0.061018 & 0.075208 & 39 & 0.087662312 & 0.087532 & 0.093708 \\
\hline 10 & & 0.124418 & 0.13351 & 40 & & 0.116055 & 0.120104 \\
\hline 11 & 0.06590498 & 0.077448 & 0.085257 & 41 & 0.089752895 & 0.090594 & 0.094549 \\
\hline 12 & 0.059514731 & 0.06882 & 0.075595 & 42 & 0.090064921 & 0.090866 & 0.094732 \\
\hline 13 & 0.067934571 & 0.066873 & 0.073799 & 43 & & 0.067801 & 0.068925 \\
\hline 14 & & 0.118421 & 0.122941 & 44 & & 0.06475 & 0.064766 \\
\hline 15 & 0.074920565 & 0.071747 & 0.076717 & 45 & & 0.007744 & 0.005192 \\
\hline 16 & & 0.107351 & 0.110228 & 46 & 0.110560216 & 0.08967 & 0.089439 \\
\hline 17 & & 0.084982 & 0.086239 & 47 & 0.092698776 & 0.078504 & 0.07985 \\
\hline 18 & 0.088947398 & 0.080672 & 0.083103 & 48 & & 0.05524 & 0.055067 \\
\hline 19 & 0.071689058 & 0.068912 & 0.071652 & 49 & & & 0.036358 \\
\hline 20 & 0.08238714 & 0.080027 & 0.083029 & 50 & & & 0.064725 \\
\hline 21 & & -0.00629 & -0.00879 & 51 & & & 0.041272 \\
\hline 22 & 0.08181357 & 0.066579 & 0.066239 & 52 & & & 0.063978 \\
\hline 23 & 0.084202241 & 0.066999 & 0.068569 & 53 & & & 0.055977 \\
\hline 24 & 0.071793781 & 0.064309 & 0.066712 & 54 & & & 0.05301 \\
\hline 25 & & 0.036758 & 0.035477 & 55 & & & 0.036875 \\
\hline 26 & 0.061867439 & 0.06349 & 0.061979 & 56 & & & 0.051251 \\
\hline 27 & 0.089388621 & 0.065327 & 0.066489 & 57 & & & 0.003508 \\
\hline 28 & & 0.087317 & 0.087437 & 58 & & & 0.089645 \\
\hline 29 & 0.069609018 & 0.070548 & 0.070535 & 59 & & & 0.062765 \\
\hline 30 & 0.079455412 & 0.073893 & 0.074498 & 60 & & & 0.053169 \\
\hline
\end{tabular}

Table 4.1.3 Forecasting fraction non-conforming for data generated using $\operatorname{AR}(1)$ process (18 observations missing) 


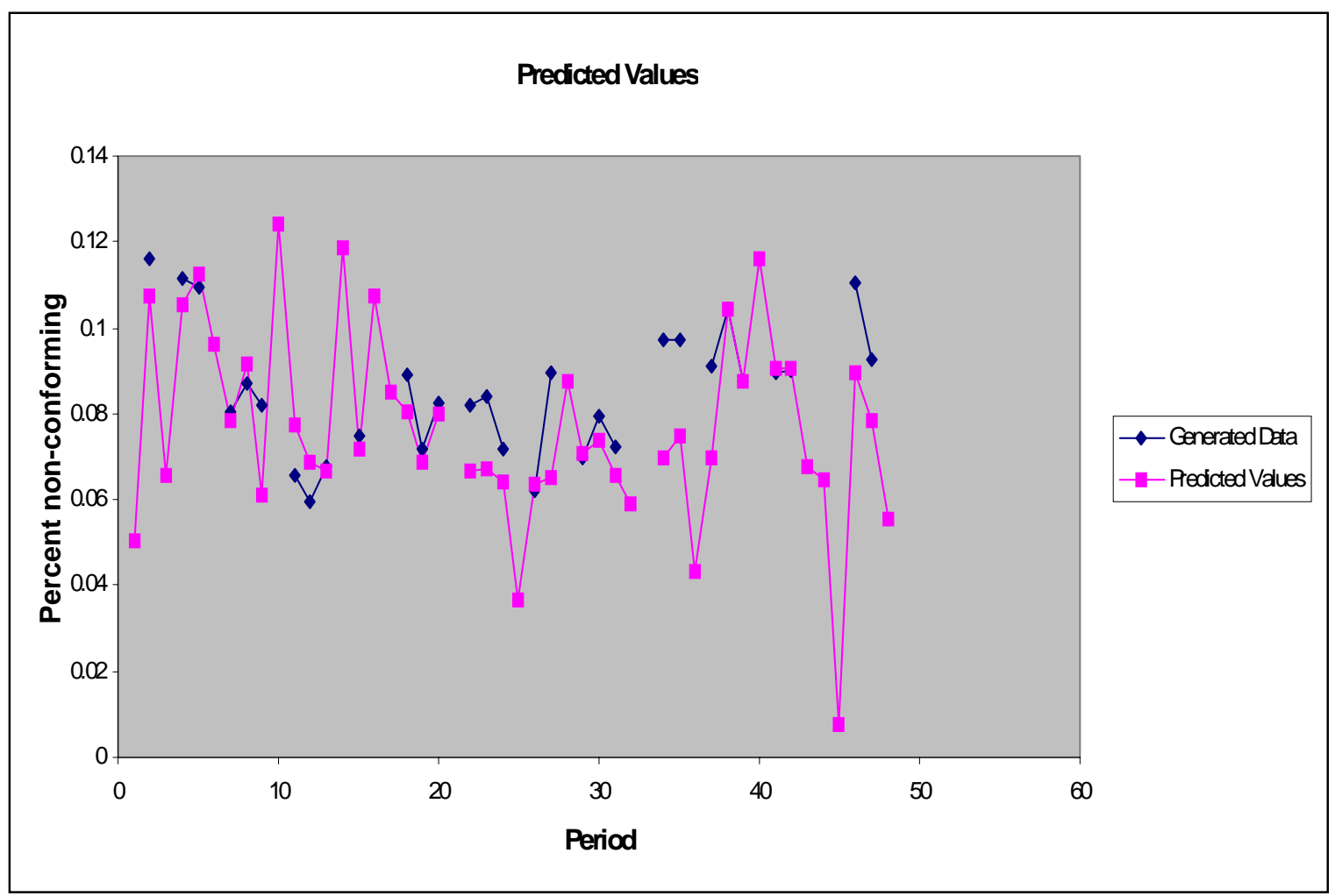

Figure 4.1.3(a) Generated vs. Predicted values, AR(1) process, 18 observations missing



Figure 4.1.3(b) Generated vs. Forecasted values, AR(1) process, 18 observations missing 


\begin{tabular}{|c|c|c|c|c|c|c|c|}
\hline Period & 11 values missing & Estimate & Forecast & Period & 11 values missing & Estimate & Forecast \\
\hline 1 & 0.1642 & 0.162503 & 0.16544 & 31 & 5343 & 0.106995 & 0.115427 \\
\hline 2 & 0.1565 & 0.157247 & 0.1601 & 32 & 0.119512935 & 0.105943 & 0.115883 \\
\hline 3 & 0.168425324 & 0.168757 & 0.171574 & 33 & 0.118884858 & 0.112204 & 0.122886 \\
\hline 4 & & 0.145005 & 0.146763 & 34 & & 0.082601 & 0.089223 \\
\hline 5 & 0.159723293 & 0.136135 & 0.140514 & 35 & 0.123152657 & 0.100262 & 0.109428 \\
\hline 6 & 0.155129697 & 0.138934 & 0.145113 & 36 & 0.122215973 & 0.106982 & 0.11784 \\
\hline 7 & 0.161908339 & 0.152652 & 0.15986 & 37 & 0.137968606 & 0.125489 & 0.137734 \\
\hline 8 & 0.154414864 & 0.146455 & 0.154547 & 38 & & 0.098164 & 0.106157 \\
\hline 9 & 0.147746925 & 0.147105 & 0.150268 & 39 & 0.111964215 & 0.107014 & 0.115709 \\
\hline 10 & 0.139014334 & 0.136783 & 0.140194 & 40 & 0.129786853 & 0.121201 & 0.131115 \\
\hline 11 & 0.129620493 & 0.130856 & 0.134129 & 41 & & 0.106477 & 0.114268 \\
\hline 12 & & 0.107313 & 0.109812 & 42 & 0.127637061 & 0.11241 & 0.121892 \\
\hline 13 & 0.127747695 & 0.105771 & 0.110712 & 43 & & 0.102182 & 0.109879 \\
\hline 14 & & 0.06781 & 0.06878 & 44 & 0.130162518 & 0.110161 & 0.120081 \\
\hline 15 & 0.140545734 & 0.10758 & 0.112212 & 45 & 0.146261316 & 0.12804 & 0.139984 \\
\hline 16 & 0.141163464 & 0.124733 & 0.131696 & 46 & 0.129851493 & 0.122716 & 0.136019 \\
\hline 17 & & 0.10054 & 0.104468 & 47 & 0.13845949 & 0.139247 & 0.147463 \\
\hline 18 & 0.123496584 & 0.103044 & 0.109244 & 48 & 0.131770323 & 0.132435 & 0.140577 \\
\hline 19 & 0.135975827 & 0.120456 & 0.12838 & 49 & & & 0.139801 \\
\hline 20 & 0.116196971 & 0.108849 & 0.11759 & 50 & & & 0.130379 \\
\hline 21 & 0.131808538 & 0.121991 & 0.131823 & 51 & & & 0.162865 \\
\hline 22 & & 0.085932 & 0.091169 & 52 & & & 0.169321 \\
\hline 23 & 0.11556988 & 0.092192 & 0.100027 & 53 & & & 0.166315 \\
\hline 24 & 0.107286341 & 0.098385 & 0.107482 & 54 & & & 0.173415 \\
\hline 25 & 0.101427086 & 0.10222 & 0.106229 & 55 & & & 0.168077 \\
\hline 26 & 0.123238537 & 0.112383 & 0.122931 & 56 & & & 0.175904 \\
\hline 27 & & 0.123847 & 0.131185 & 57 & & & 0.176758 \\
\hline 28 & & 0.093723 & 0.099209 & 58 & & & 0.159041 \\
\hline 29 & 0.108545276 & 0.097417 & 0.104481 & 59 & & & 0.176286 \\
\hline 30 & 0.09555355 & 0.092112 & 0.099558 & 60 & & & 0.170138 \\
\hline
\end{tabular}

Table 4.2.1 Forecasting fraction non-conforming for data generated using $\operatorname{AR}(2)$ process (11) observations missing 


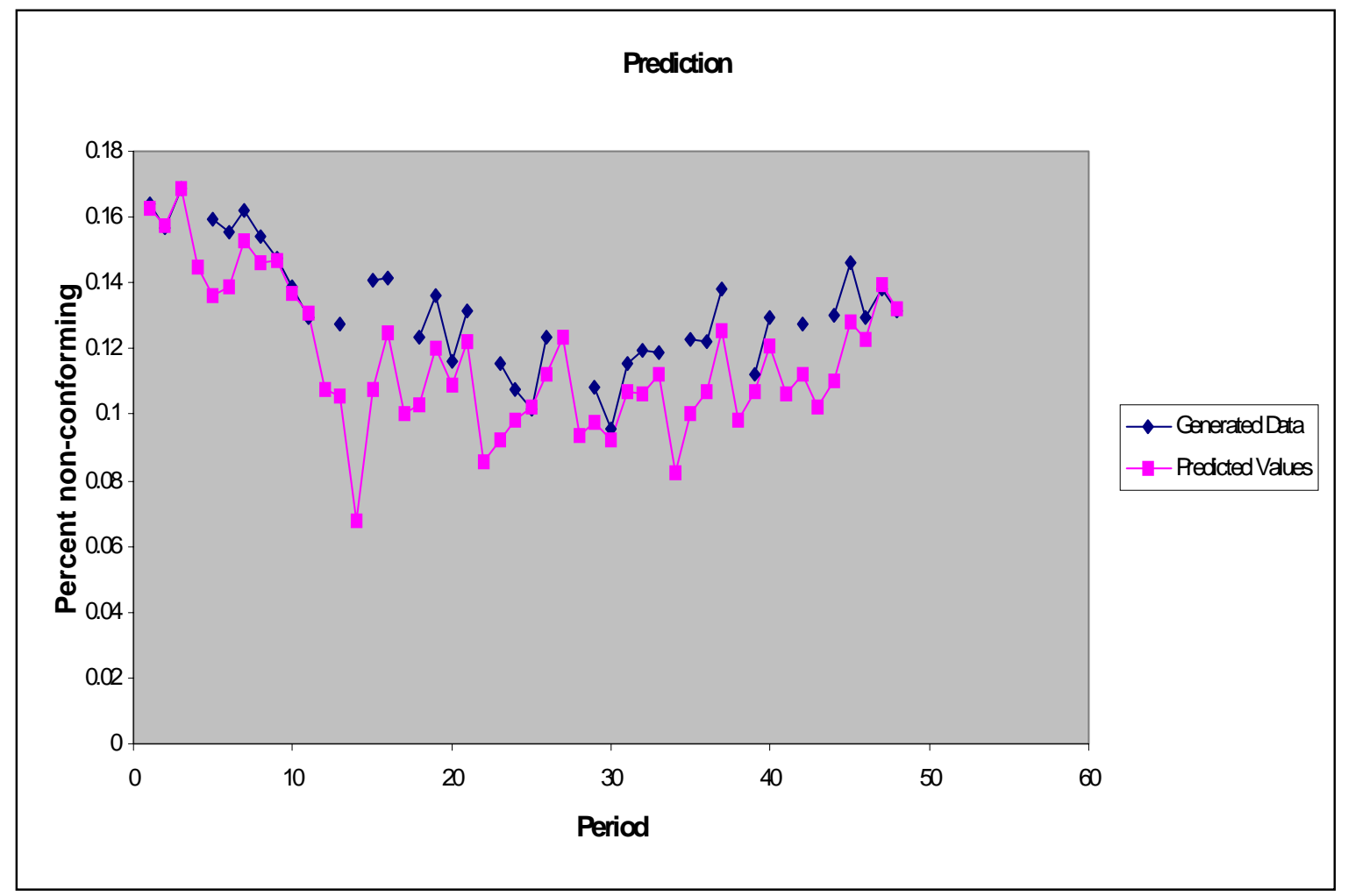

Figure 4.2.1(a) Generated vs. Predicted values, AR(2) process, 11 observations missing

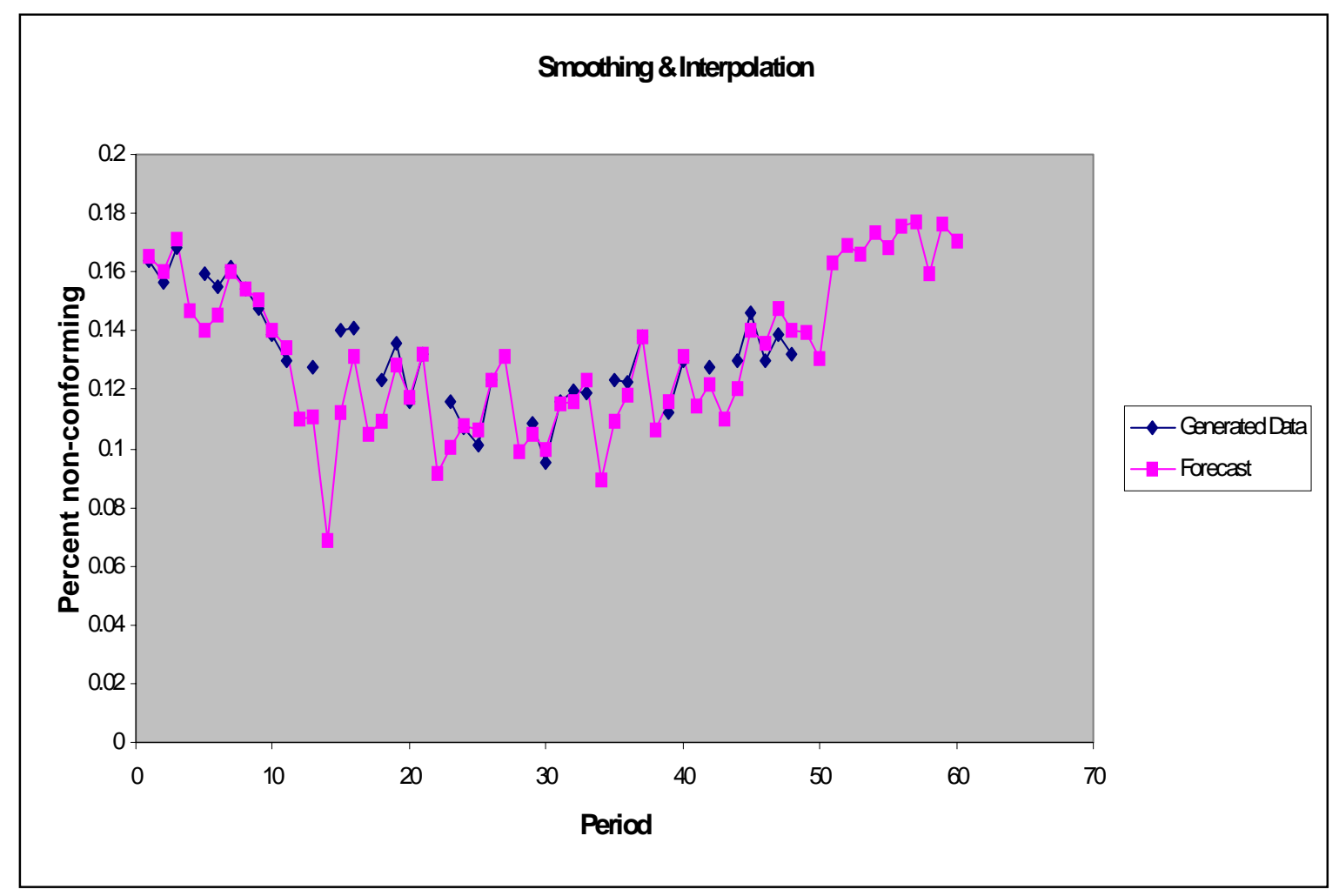

Figure 4.2.1(b) Generated vs. Forecasted values, $\operatorname{AR}(2)$ process, 11 observations missing 


\begin{tabular}{|c|c|c|c|c|c|c|c|}
\hline Period & 13 values missing & Estimate & Forecast & Period & 13 values missing & Estimate & Forecast \\
\hline 1 & 0.1642 & 0.162503 & 0.17044 & 31 & & 0.092914 & 0.098979 \\
\hline 2 & 0.1565 & 0.157247 & 0.1651 & 32 & 0.119512935 & 0.097494 & 0.106005 \\
\hline 3 & 0.168425324 & 0.168757 & 0.176574 & 33 & 0.118884858 & 0.106994 & 0.116826 \\
\hline 4 & 0.170067154 & 0.170107 & 0.177919 & 34 & & 0.074644 & 0.080647 \\
\hline 5 & 0.159723293 & 0.160108 & 0.167878 & 35 & 0.123152657 & 0.098089 & 0.106877 \\
\hline 6 & 0.155129697 & 0.154539 & 0.162374 & 36 & & 0.065109 & 0.071076 \\
\hline 7 & 0.161908339 & 0.162611 & 0.170368 & 37 & 0.137968606 & 0.106497 & 0.115961 \\
\hline 8 & 0.154414864 & 0.152629 & 0.160584 & 38 & & 0.068802 & 0.075421 \\
\hline 9 & 0.147746925 & 0.15076 & 0.158381 & 39 & 0.111964215 & 0.100805 & 0.109007 \\
\hline 10 & 0.139014334 & 0.138781 & 0.146427 & 40 & & 0.076426 & 0.083274 \\
\hline 11 & 0.129620493 & 0.131779 & 0.139186 & 41 & & 0.049977 & 0.05571 \\
\hline 12 & & 0.107877 & 0.109483 & 42 & 0.127637061 & 0.0965 & 0.105693 \\
\hline 13 & 0.127747695 & 0.105595 & 0.109662 & 43 & & 0.078233 & 0.085916 \\
\hline 14 & & 0.066829 & 0.066971 & 44 & 0.130162518 & 0.105645 & 0.116053 \\
\hline 15 & 0.140545734 & 0.10703 & 0.110896 & 45 & 0.146261316 & 0.125102 & 0.13786 \\
\hline 16 & 0.141163464 & 0.11992 & 0.126147 & 46 & 0.129851493 & 0.121361 & 0.135737 \\
\hline 17 & & 0.080218 & 0.082855 & 47 & & 0.125096 & 0.135745 \\
\hline 18 & 0.123496584 & 0.096074 & 0.101758 & 48 & 0.131770323 & 0.117989 & 0.130593 \\
\hline 19 & 0.135975827 & 0.117443 & 0.125186 & 49 & & & 0.132431 \\
\hline 20 & 0.116196971 & 0.107884 & 0.116551 & 50 & & & 0.109255 \\
\hline 21 & 0.131808538 & 0.121989 & 0.131747 & 51 & & & 0.14848 \\
\hline 22 & & 0.08637 & 0.091385 & 52 & & & 0.133921 \\
\hline 23 & 0.11556988 & 0.092209 & 0.099819 & 53 & & & 0.125815 \\
\hline 24 & 0.107286341 & 0.098503 & 0.107359 & 54 & & & 0.151302 \\
\hline 25 & 0.101427086 & 0.102266 & 0.111029 & 55 & & & 0.13954 \\
\hline 26 & 0.123238537 & 0.112302 & 0.122616 & 56 & & & 0.156629 \\
\hline 27 & & 0.123932 & 0.131065 & 57 & & & 0.154771 \\
\hline 28 & 0.100639538 & 0.099475 & 0.106738 & 58 & & & 0.134704 \\
\hline 29 & 0.108545276 & 0.098914 & 0.107543 & 59 & & & 0.151574 \\
\hline 30 & 0.09555355 & 0.097503 & 0.105916 & 60 & & & 0.149234 \\
\hline
\end{tabular}

Table 4.2.2 Forecasting fraction non-conforming for data generated using $\operatorname{AR}(2)$ process (13 observations missing) 


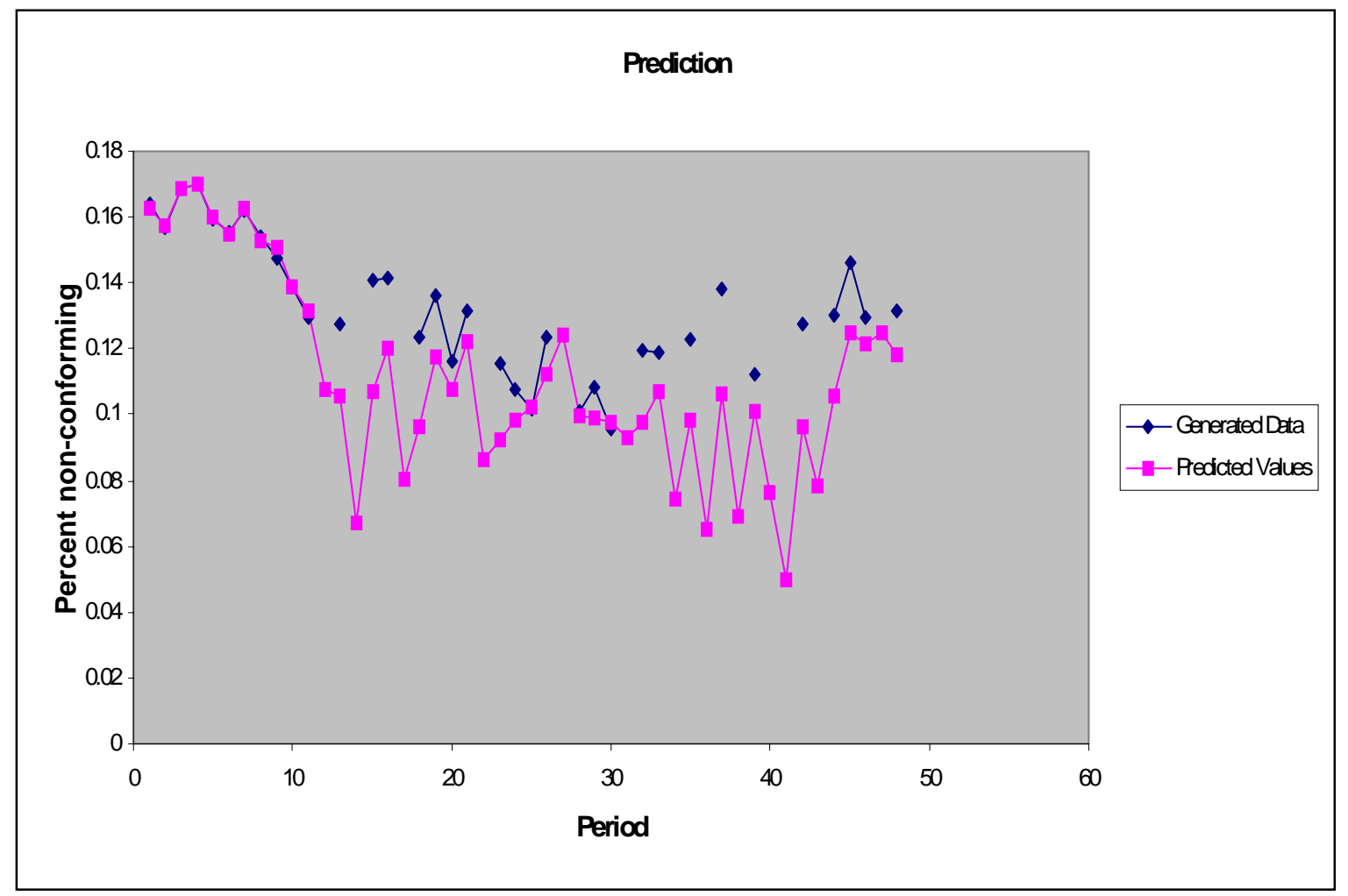

Figure 4.2.2(a) Generated vs. Predicted values, AR(2) process, 13 observations missing

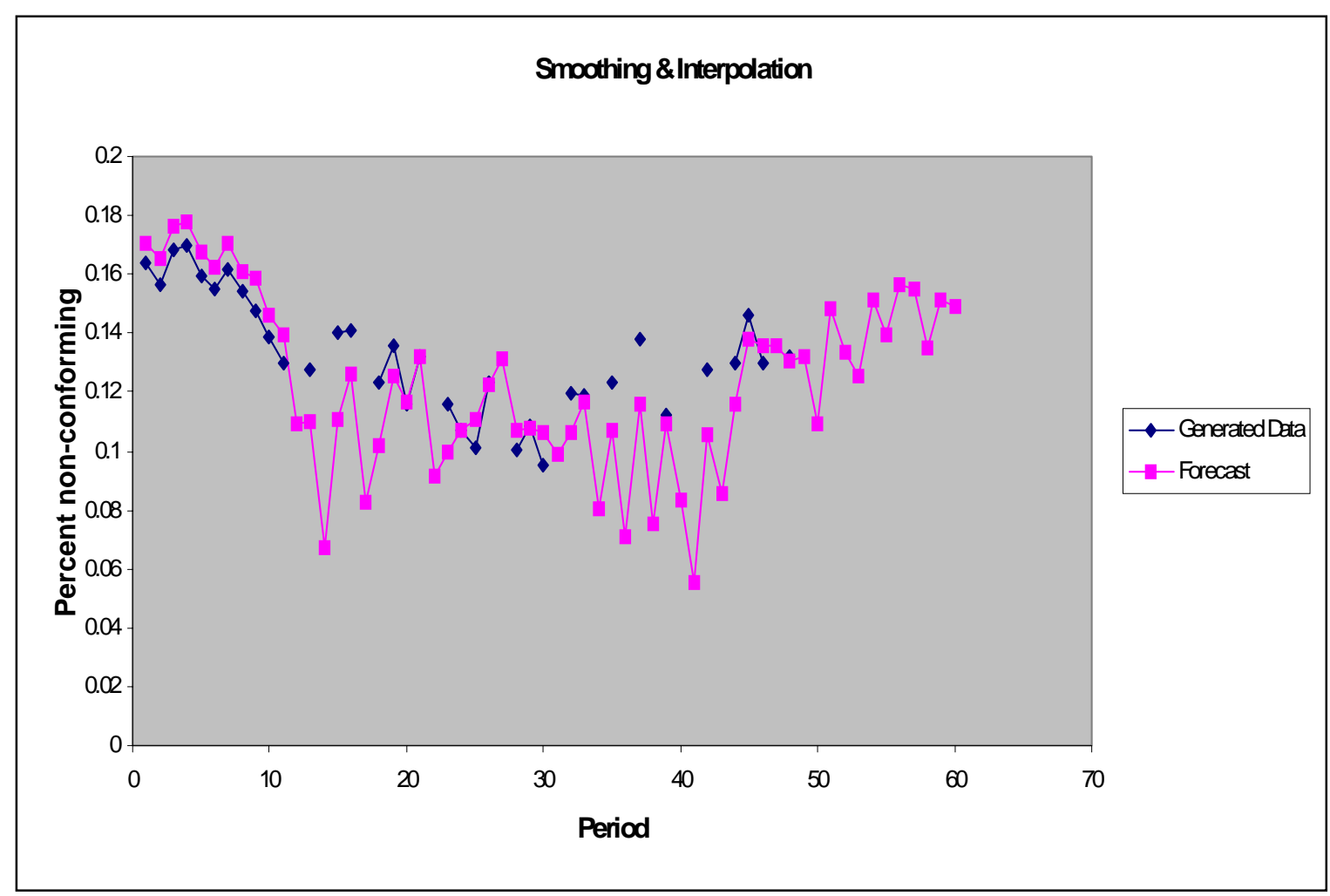

Figure 4.2.2(b) Generated vs. Predicted values, AR(2) process, 13 observations missing 


\begin{tabular}{|c|c|c|c|c|c|c|c|}
\hline Period & 16 values missing & Estimate & Forecast & Period & $\begin{array}{l}16 \text { values } \\
\text { missing }\end{array}$ & Estimate & Forecast \\
\hline 1 & 0.1642 & 0.162503 & 0.17044 & 31 & & 0.082534 & 0.093865 \\
\hline 2 & 0.1565 & 0.157247 & 0.1651 & 32 & 0.119512935 & 0.095457 & 0.10946 \\
\hline 3 & 0.168425324 & 0.168757 & 0.176574 & 33 & 0.118884858 & 0.105576 & 0.121058 \\
\hline 4 & & 0.145005 & 0.151763 & 34 & & 0.072276 & 0.084149 \\
\hline 5 & 0.159723293 & 0.136135 & 0.145514 & 35 & 0.123152657 & 0.097923 & 0.112599 \\
\hline 6 & 0.155129697 & 0.138934 & 0.150113 & 36 & & 0.065251 & 0.077112 \\
\hline 7 & 0.161908339 & 0.152652 & 0.16486 & 37 & 0.137968606 & 0.113547 & 0.128872 \\
\hline 8 & 0.154414864 & 0.146455 & 0.154547 & 38 & & 0.083404 & 0.096286 \\
\hline 9 & 0.147746925 & 0.147105 & 0.155268 & 39 & 0.111964215 & 0.102606 & 0.116816 \\
\hline 10 & 0.139014334 & 0.136783 & 0.145194 & 40 & & 0.087555 & 0.100339 \\
\hline 11 & 0.129620493 & 0.130856 & 0.139129 & 41 & & 0.078506 & 0.090784 \\
\hline 12 & & 0.107313 & 0.114812 & 42 & 0.127637061 & 0.105052 & 0.11984 \\
\hline 13 & 0.127747695 & 0.105771 & 0.115712 & 43 & & 0.087064 & 0.100361 \\
\hline 14 & & 0.06781 & 0.07378 & 44 & 0.130162518 & 0.109171 & 0.124801 \\
\hline 15 & 0.140545734 & 0.10758 & 0.117212 & 45 & 0.146261316 & 0.126776 & 0.14457 \\
\hline 16 & 0.141163464 & 0.124733 & 0.136696 & 46 & 0.129851493 & 0.121524 & 0.140905 \\
\hline 17 & & 0.10054 & 0.109468 & 47 & & 0.125228 & 0.140946 \\
\hline 18 & 0.123496584 & 0.103044 & 0.114244 & 48 & 0.131770323 & 0.117984 & 0.135658 \\
\hline 19 & 0.135975827 & 0.120456 & 0.13338 & 49 & & & 0.138797 \\
\hline 20 & 0.116196971 & 0.108849 & 0.11759 & 50 & & & 0.120772 \\
\hline 21 & 0.131808538 & 0.121991 & 0.131823 & 51 & & & 0.146147 \\
\hline 22 & & 0.085932 & 0.096169 & 52 & & & 0.137483 \\
\hline 23 & 0.11556988 & 0.092192 & 0.105027 & 53 & & & 0.146681 \\
\hline 24 & 0.107286341 & 0.098385 & 0.107482 & 54 & & & 0.156563 \\
\hline 25 & & 0.089996 & 0.100685 & 55 & & & 0.144516 \\
\hline 26 & 0.123238537 & 0.102084 & 0.115774 & 56 & & & 0.159525 \\
\hline 27 & & 0.10388 & 0.115309 & 57 & & & 0.157522 \\
\hline 28 & & 0.08098 & 0.091164 & 58 & & & 0.136288 \\
\hline 29 & 0.108545276 & 0.094765 & 0.106904 & 59 & & & 0.152936 \\
\hline 30 & 0.09555355 & 0.090107 & 0.097851 & 60 & & & 0.150068 \\
\hline
\end{tabular}

Table 4.2.3 Forecasting fraction non-conforming for data generated using $\operatorname{AR}(2)$ process (16 observations missing) 


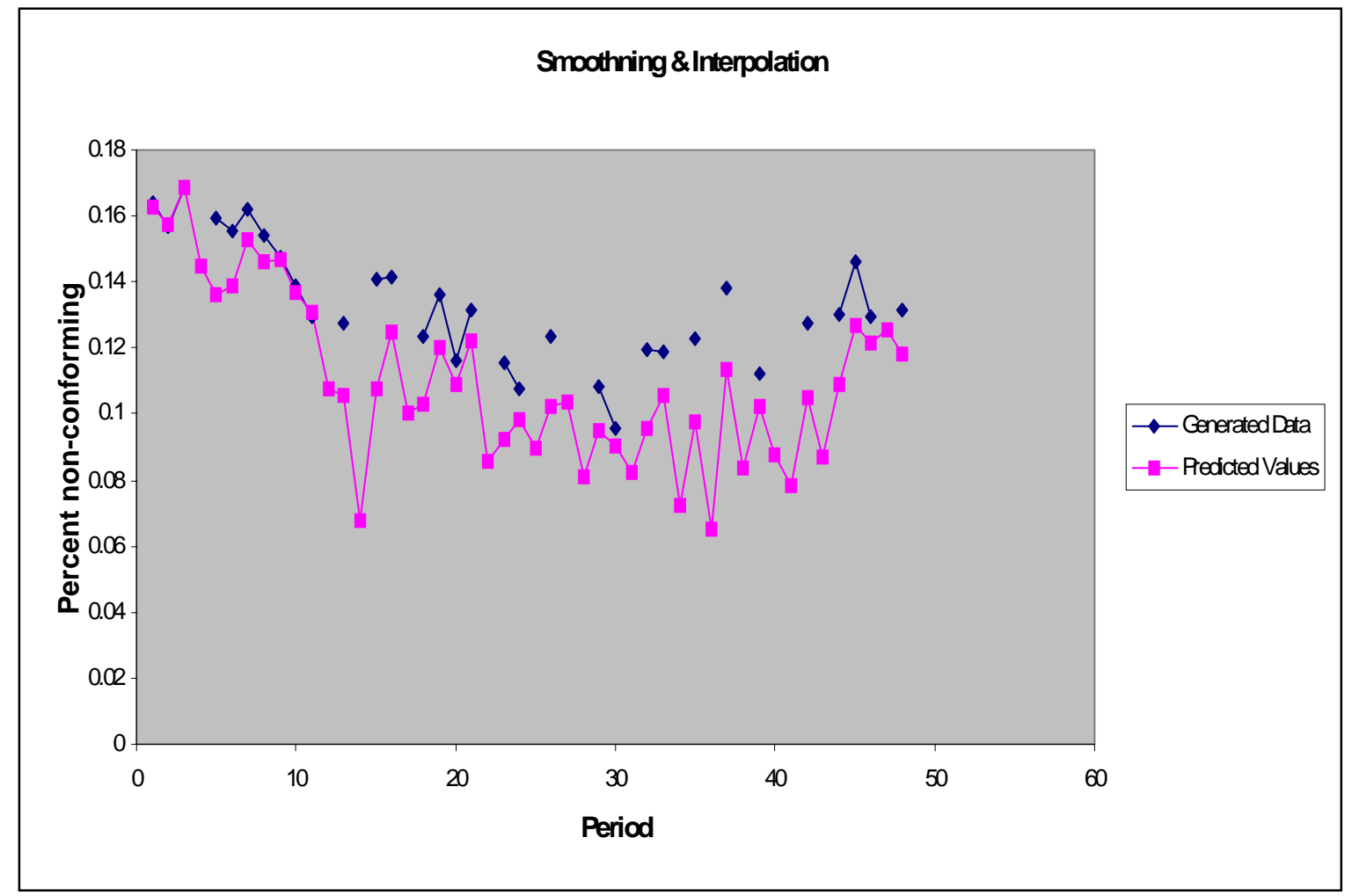

Figure 4.2.3(a) Generated vs. Predicted values, AR(2) process, 16 observations missing

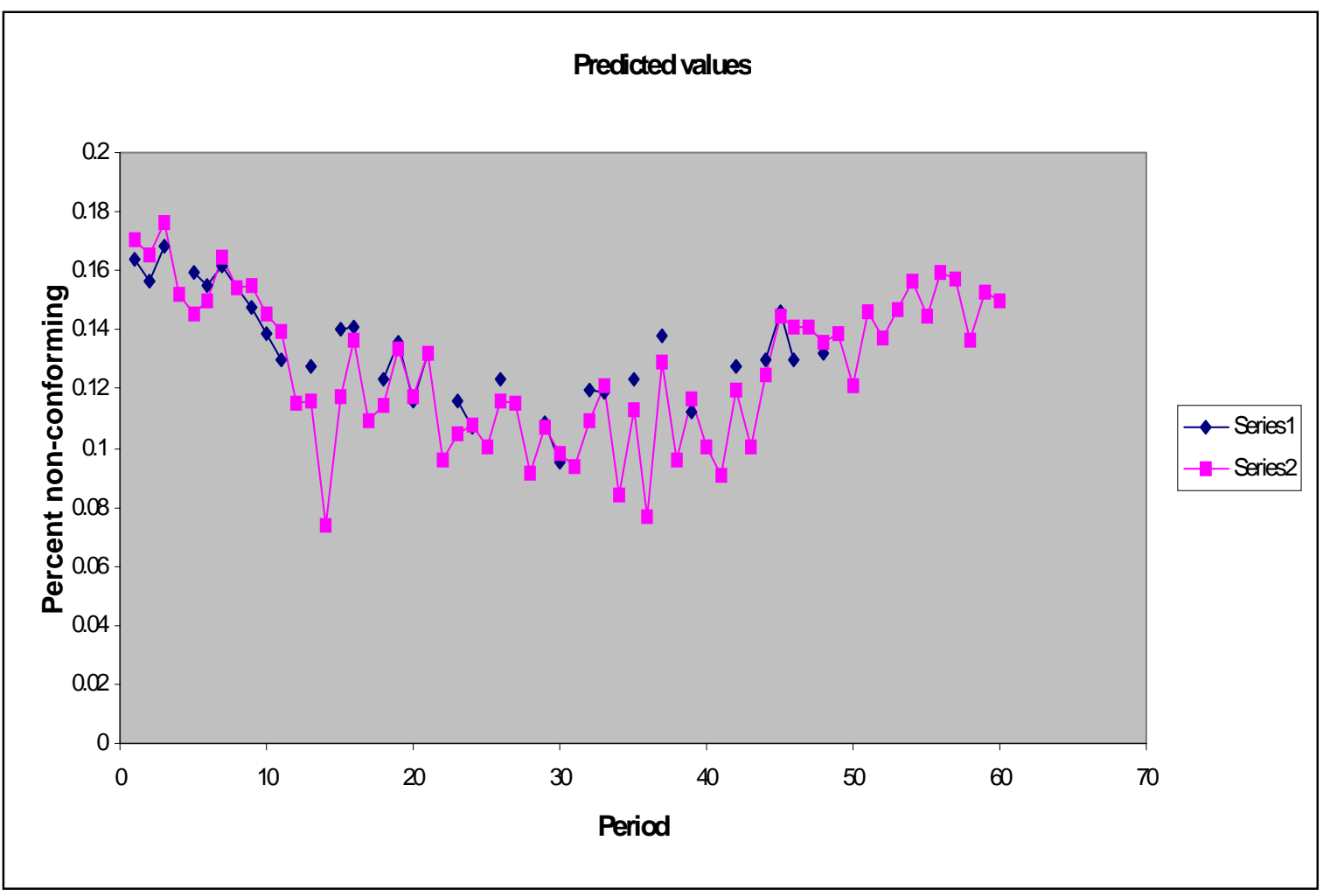

Figure 4.2.3(b) Generated vs. Predicted values, AR(2) process, 16 observations missing 


\begin{tabular}{|c|c|c|c|c|c|c|c|}
\hline Period & 18 values missing & Estimate & Forecast & Period & 18 values missing & Estimate & Forecast \\
\hline 1 & 0.1642 & 0.162503 & 0.17044 & 31 & & 0.083852 & 0.090932 \\
\hline 2 & 0.1565 & 0.157247 & 0.1651 & 32 & 0.119512935 & 0.121127 & 0.128028 \\
\hline 3 & 0.168425324 & 0.168757 & 0.176574 & 33 & 0.118884858 & 0.103584 & 0.112184 \\
\hline 4 & & 0.145005 & 0.146763 & 34 & & 0.058293 & 0.064869 \\
\hline 5 & 0.159723293 & 0.136135 & 0.140514 & 35 & 0.123152657 & 0.096683 & 0.106201 \\
\hline 6 & 0.155129697 & 0.138934 & 0.145113 & 36 & & 0.060219 & 0.067552 \\
\hline 7 & 0.161908339 & 0.152652 & 0.15986 & 37 & 0.137968606 & 0.112911 & 0.123798 \\
\hline 8 & & 0.104173 & 0.105746 & 38 & & 0.081438 & 0.09005 \\
\hline 9 & 0.147746925 & 0.121293 & 0.125806 & 39 & 0.111964215 & 0.102509 & 0.112462 \\
\hline 10 & 0.139014334 & 0.119982 & 0.141609 & 40 & & 0.087688 & 0.096231 \\
\hline 11 & 0.129620493 & 0.120133 & 0.132814 & 41 & & 0.078546 & 0.086531 \\
\hline 12 & & 0.091803 & 0.094449 & 42 & 0.127637061 & 0.105151 & 0.115633 \\
\hline 13 & 0.127747695 & 0.101836 & 0.107361 & 43 & & 0.088056 & 0.096971 \\
\hline 14 & & 0.062791 & 0.064583 & 44 & 0.130162518 & 0.128511 & 0.137609 \\
\hline 15 & 0.140545734 & 0.106586 & 0.112151 & 45 & & 0.094463 & 0.103411 \\
\hline 16 & 0.141163464 & 0.124529 & 0.142454 & 46 & 0.129851493 & 0.113665 & 0.130696 \\
\hline 17 & & 0.100984 & 0.105853 & 47 & & 0.106883 & 0.117103 \\
\hline 18 & 0.123496584 & 0.103497 & 0.125588 & 48 & 0.131770323 & 0.114065 & 0.126796 \\
\hline 19 & 0.135975827 & 0.121048 & 0.129798 & 49 & & & 0.129754 \\
\hline 20 & 0.116196971 & 0.139247 & 0.144728 & 50 & & & 0.109225 \\
\hline 21 & 0.131808538 & 0.112954 & 0.120529 & 51 & & & 0.134651 \\
\hline 22 & & 0.069924 & 0.074733 & 52 & & & 0.125501 \\
\hline 23 & 0.11556988 & 0.088767 & 0.096554 & 53 & & & 0.134 \\
\hline 24 & 0.107286341 & 0.094746 & 0.114312 & 54 & & & 0.143713 \\
\hline 25 & & 0.08693 & 0.093483 & 55 & & & 0.132081 \\
\hline 26 & 0.123238537 & 0.101393 & 0.126046 & 56 & & & 0.186982 \\
\hline 27 & & 0.104223 & 0.111661 & 57 & & & 0.157166 \\
\hline 28 & & 0.082062 & 0.088226 & 58 & & & 0.150217 \\
\hline 29 & 0.108545276 & 0.095231 & 0.113284 & 59 & & & 0.151383 \\
\hline 30 & 0.09555355 & 0.090685 & 0.099278 & 60 & & & 0.146836 \\
\hline
\end{tabular}

Table 4.2.4 Forecasting fraction non-conforming for data generated using $\operatorname{AR}(2)$ process (18 observations missing) 


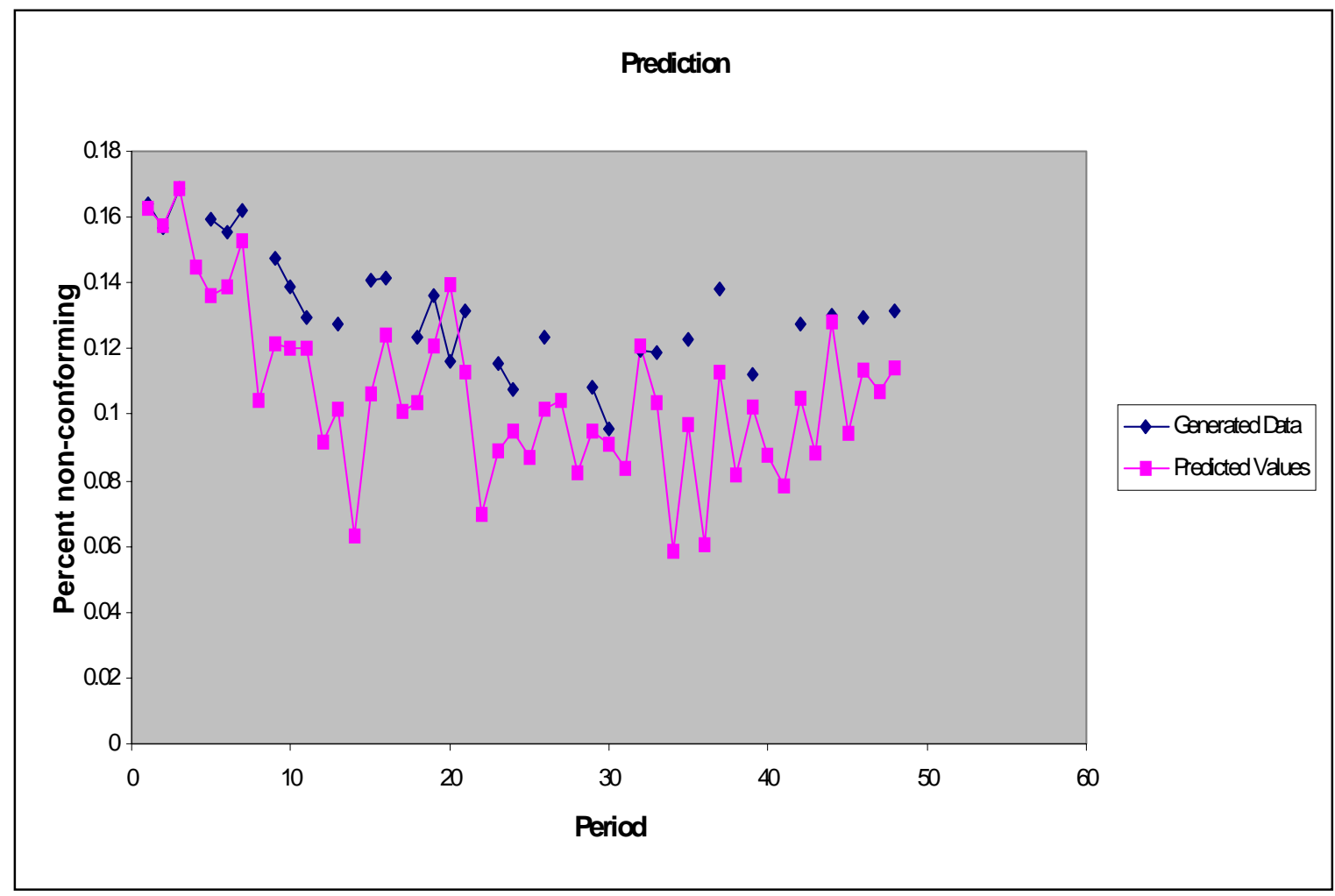

Figure 4.2.4(a) Generated vs. Predicted values, AR(2) process, 18 observations missing

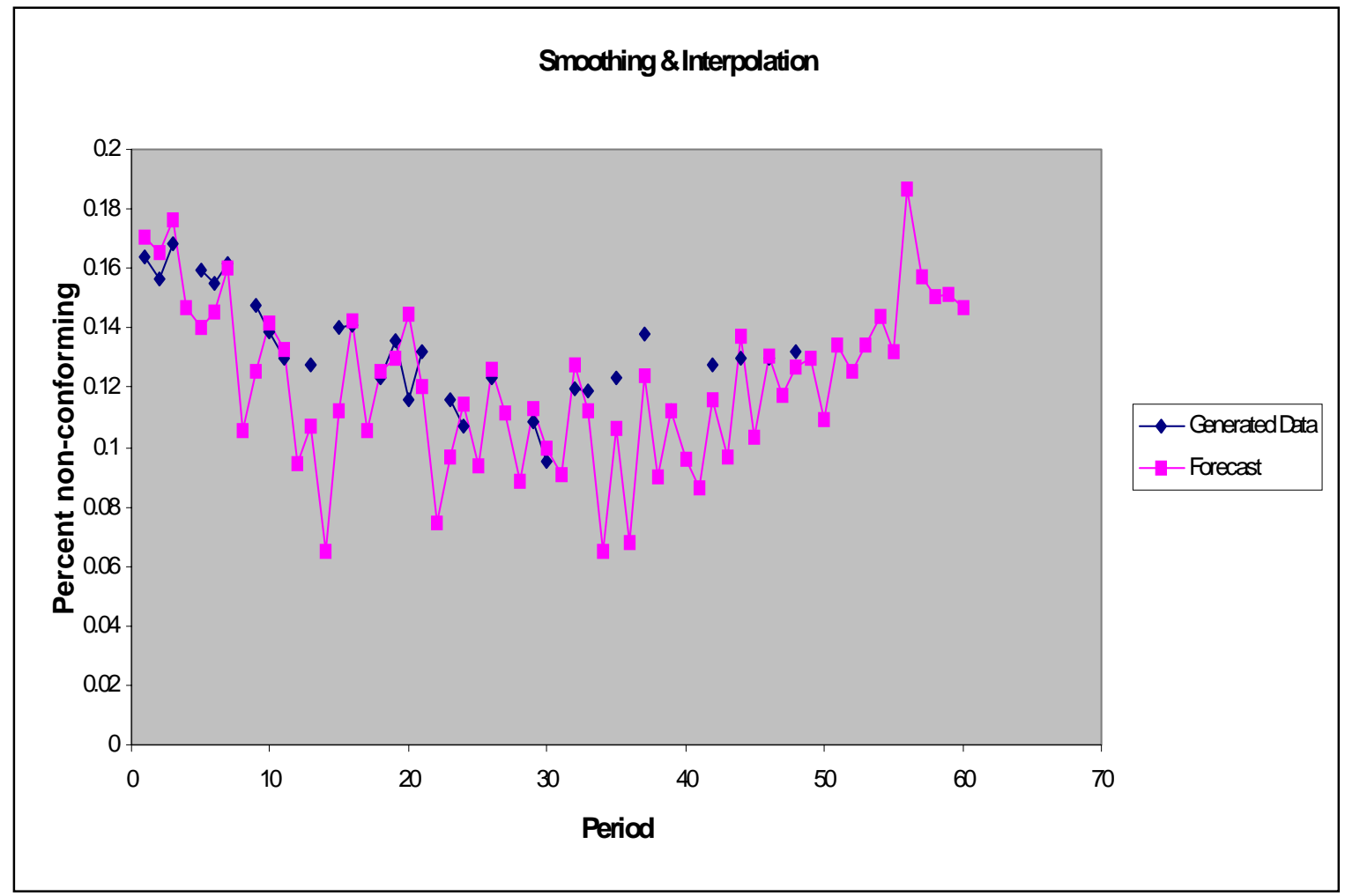

Figure 4.2.4(b) Generated vs. Forecasted values, $\operatorname{AR}(2)$ process, 18 observations missing 


\begin{tabular}{|c|c|c|c|c|c|c|c|}
\hline Period & 10 obs. Missing & Estimate & Forecast & Period & 10 obs. Missing & Estimate & Forecast \\
\hline 1 & 0.1465 & 0.148913 & 0.147766 & 31 & 0.138325026 & 0.127773 & 0.13203 \\
\hline 2 & 0.130053586 & 0.131088 & 0.134826 & 32 & & 0.10357 & 0.104703 \\
\hline 3 & 0.138678662 & 0.138381 & 0.142153 & 33 & 0.15460471 & 0.126486 & 0.130744 \\
\hline 4 & 0.157103537 & 0.158063 & 0.161728 & 34 & 0.162430559 & 0.140254 & 0.166976 \\
\hline 5 & 0.153766381 & 0.151151 & 0.155106 & 35 & 0.162554885 & 0.1518 & 0.159717 \\
\hline 6 & & 0.12782 & 0.1271 & 36 & 0.164281013 & 0.155009 & 0.168956 \\
\hline 7 & 0.138591559 & 0.120463 & 0.121758 & 37 & 0.169349983 & 0.166638 & 0.175886 \\
\hline 8 & 0.137380734 & 0.125169 & 0.137821 & 38 & 0.173767257 & 0.168429 & 0.17827 \\
\hline 9 & 0.118681376 & 0.112171 & 0.120546 & 39 & & 0.149667 & 0.153432 \\
\hline 10 & 0.11673186 & 0.113595 & 0.117319 & 40 & 0.158672836 & 0.146661 & 0.151761 \\
\hline 11 & & 0.105432 & 0.104881 & 41 & & 0.125563 & 0.127104 \\
\hline 12 & 0.107904413 & 0.08996 & 0.091402 & 42 & 0.156703571 & 0.150393 & 0.154148 \\
\hline 13 & 0.129791784 & 0.117269 & 0.120103 & 43 & 0.166318687 & 0.152965 & 0.168203 \\
\hline 14 & 0.113370163 & 0.105398 & 0.114118 & 44 & 0.165603794 & 0.157548 & 0.164321 \\
\hline 15 & 0.119406005 & 0.11495 & 0.119165 & 45 & 0.180469625 & 0.174806 & 0.182208 \\
\hline 16 & & 0.120469 & 0.121509 & 46 & 0.172775322 & 0.169365 & 0.177146 \\
\hline 17 & 0.128191506 & 0.114073 & 0.116681 & 47 & & 0.148605 & 0.1516 \\
\hline 18 & 0.133870786 & 0.123948 & 0.137964 & 48 & 0.164728272 & 0.144262 & 0.149531 \\
\hline 19 & & 0.105905 & 0.106886 & 49 & & & 0.135555 \\
\hline 20 & & 0.071059 & 0.070174 & 50 & & & 0.127436 \\
\hline 21 & 0.117405078 & 0.088133 & 0.090501 & 51 & & & 0.128409 \\
\hline 22 & 0.122978165 & 0.102304 & 0.106969 & 52 & & & 0.150022 \\
\hline 23 & 0.126881878 & 0.11885 & 0.129654 & 53 & & & 0.14333 \\
\hline 24 & 0.124074717 & 0.117707 & 0.124219 & 54 & & & 0.171104 \\
\hline 25 & 0.116652999 & 0.122151 & 0.128052 & 55 & & & 0.160352 \\
\hline 26 & 0.127978247 & 0.120842 & 0.127536 & 56 & & & 0.149623 \\
\hline 27 & 0.136740953 & 0.132031 & 0.139248 & 57 & & & 0.159085 \\
\hline 28 & 0.14215751 & 0.144401 & 0.1513 & 58 & & & 0.147722 \\
\hline 29 & 0.139708315 & 0.143327 & 0.149823 & 59 & & & 0.143592 \\
\hline 30 & & 0.130482 & 0.133243 & 60 & & & 0.147495 \\
\hline
\end{tabular}

Table 4.3.1 Forecasting fraction non-conforming for data generated using ARMA(1,1) process (10 observations missing) 


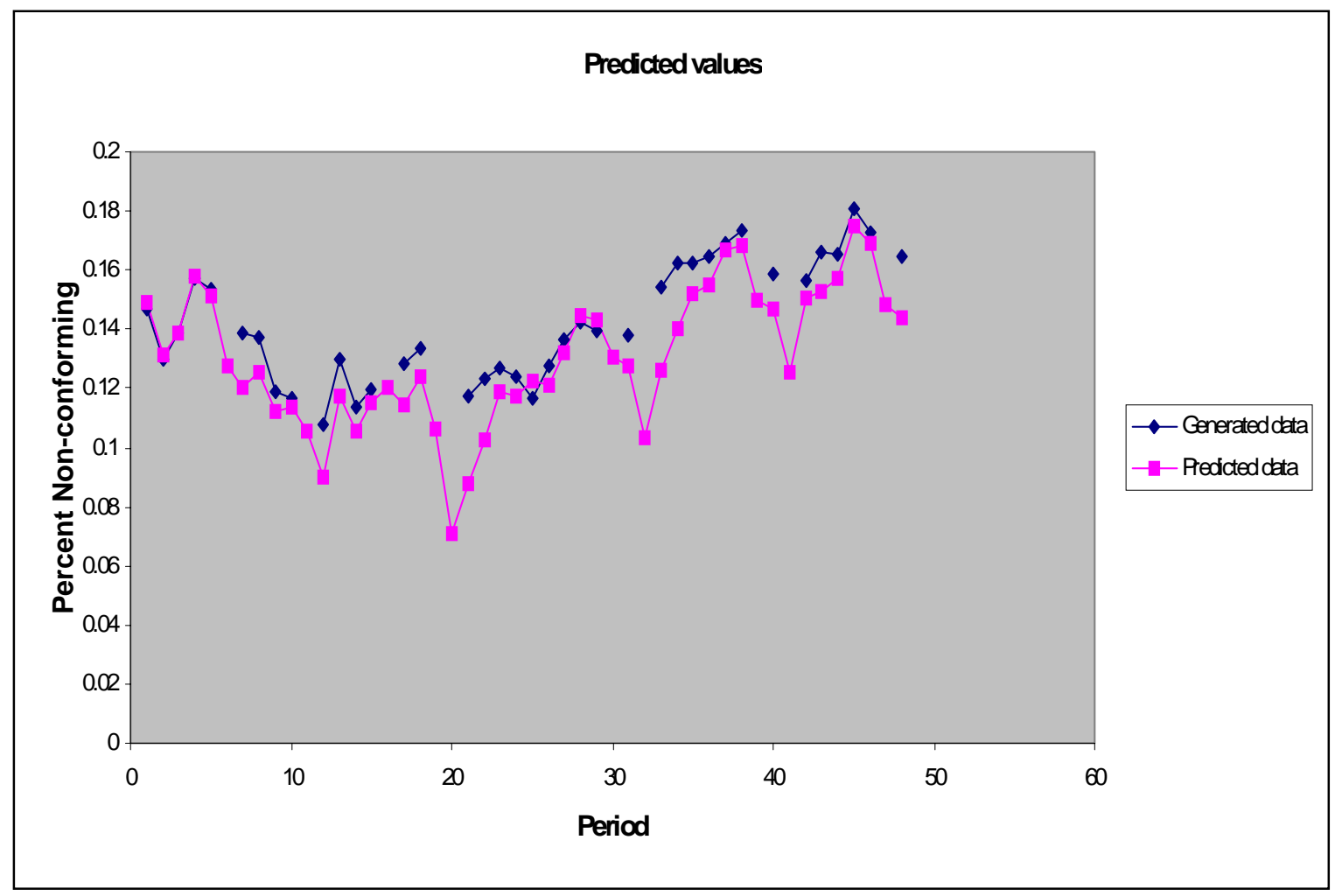

Figure 4.3.1(a) Generated vs. Predicted values, ARMA(1,1) process, 10 observations missing 


\begin{tabular}{|c|c|c|c|c|c|c|c|}
\hline Period & 11 obs. Missing & Estimate & Forecast & Period & 11 obs. Missing & Estimate & Forecast \\
\hline 1 & 0.1465 & 0.148913 & 0.153766 & 31 & 0.1383 & 0.125428 & 0.13113 \\
\hline 2 & 0.130053586 & 0.131088 & 0.135826 & 32 & & 0.099397 & 0.102157 \\
\hline 3 & 0.138678662 & 0.138381 & 0.143153 & 33 & 0.15460471 & 0.125729 & 0.131697 \\
\hline 4 & 0.157103537 & 0.158063 & 0.162728 & 34 & 0.162430559 & 0.140056 & 0.14851 \\
\hline 5 & 0.153766381 & 0.151151 & 0.156106 & 35 & 0.162554885 & 0.151944 & 0.161577 \\
\hline 6 & & 0.12782 & 0.1281 & 36 & 0.164281013 & 0.155353 & 0.165978 \\
\hline 7 & 0.138591559 & 0.120463 & 0.122758 & 37 & 0.169349983 & 0.167088 & 0.177964 \\
\hline 8 & 0.137380734 & 0.125169 & 0.128821 & 38 & 0.173767257 & 0.168633 & 0.180237 \\
\hline 9 & 0.118681376 & 0.112171 & 0.116546 & 39 & & 0.167786 & 0.173698 \\
\hline 10 & 0.11673186 & 0.113595 & 0.118319 & 40 & 0.158672836 & 0.153778 & 0.160233 \\
\hline 11 & & 0.105432 & 0.105881 & 41 & & 0.13265 & 0.135727 \\
\hline 12 & 0.107904413 & 0.08996 & 0.092402 & 42 & 0.156703571 & 0.150766 & 0.155926 \\
\hline 13 & 0.129791784 & 0.117269 & 0.121103 & 43 & 0.166318687 & 0.154769 & 0.161212 \\
\hline 14 & 0.113370163 & 0.105398 & 0.110118 & 44 & 0.165603794 & 0.157667 & 0.165623 \\
\hline 15 & 0.119406005 & 0.11495 & 0.120165 & 45 & 0.180469625 & 0.175176 & 0.183719 \\
\hline 16 & & 0.120469 & 0.122509 & 46 & 0.172775322 & 0.169504 & 0.178411 \\
\hline 17 & 0.128191506 & 0.114073 & 0.117681 & 47 & & 0.148682 & 0.15279 \\
\hline 18 & 0.133870786 & 0.123948 & 0.128964 & 48 & 0.164728272 & 0.144255 & 0.150638 \\
\hline 19 & & 0.105905 & 0.107886 & 49 & & & 0.135587 \\
\hline 20 & & 0.071059 & 0.071174 & 50 & & & 0.125355 \\
\hline 21 & 0.117405078 & 0.088133 & 0.091501 & 51 & & & 0.142059 \\
\hline 22 & 0.122978165 & 0.102304 & 0.107969 & 52 & & & 0.157241 \\
\hline 23 & 0.126881878 & 0.11885 & 0.125654 & 53 & & & 0.149556 \\
\hline 24 & 0.124074717 & 0.117707 & 0.125219 & 54 & & & 0.170417 \\
\hline 25 & 0.116652999 & 0.122151 & 0.129052 & 55 & & & 0.162809 \\
\hline 26 & & 0.091493 & 0.094228 & 56 & & & 0.150531 \\
\hline 27 & 0.136740953 & 0.112396 & 0.117836 & 57 & & & 0.160586 \\
\hline 28 & 0.14215751 & 0.13439 & 0.140932 & 58 & & & 0.149119 \\
\hline 29 & 0.139708315 & 0.13517 & 0.142216 & 59 & & & 0.144967 \\
\hline 30 & & 0.118684 & 0.122557 & 60 & & & 0.148855 \\
\hline
\end{tabular}

Table 4.3.2 Forecasting fraction non-conforming for data generated using ARMA(1,1) process (11 observations missing) 


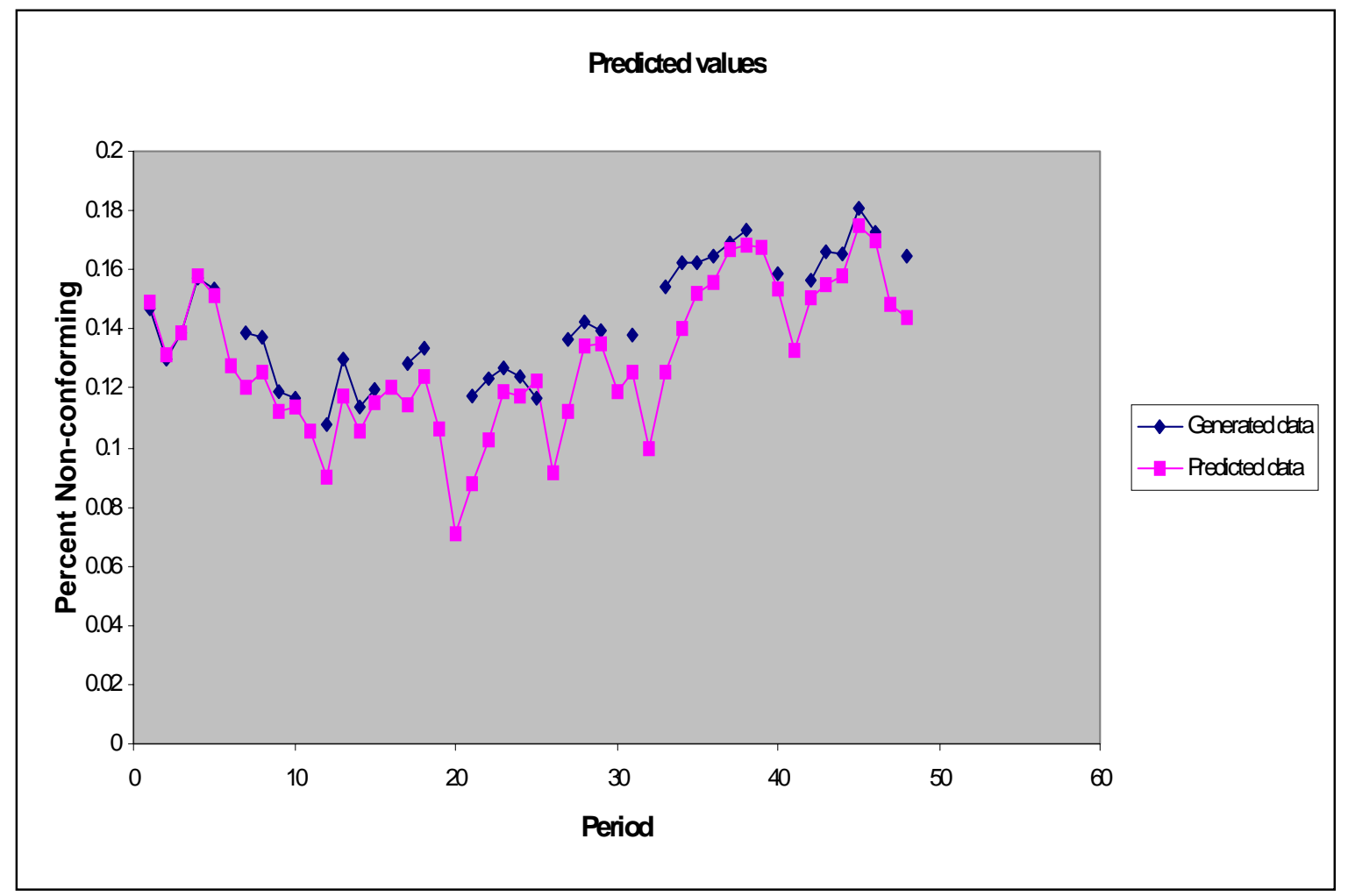

Figure 4.3.2(a) Generated vs. Predicted values, ARMA(1,1) process, 11 observations missing

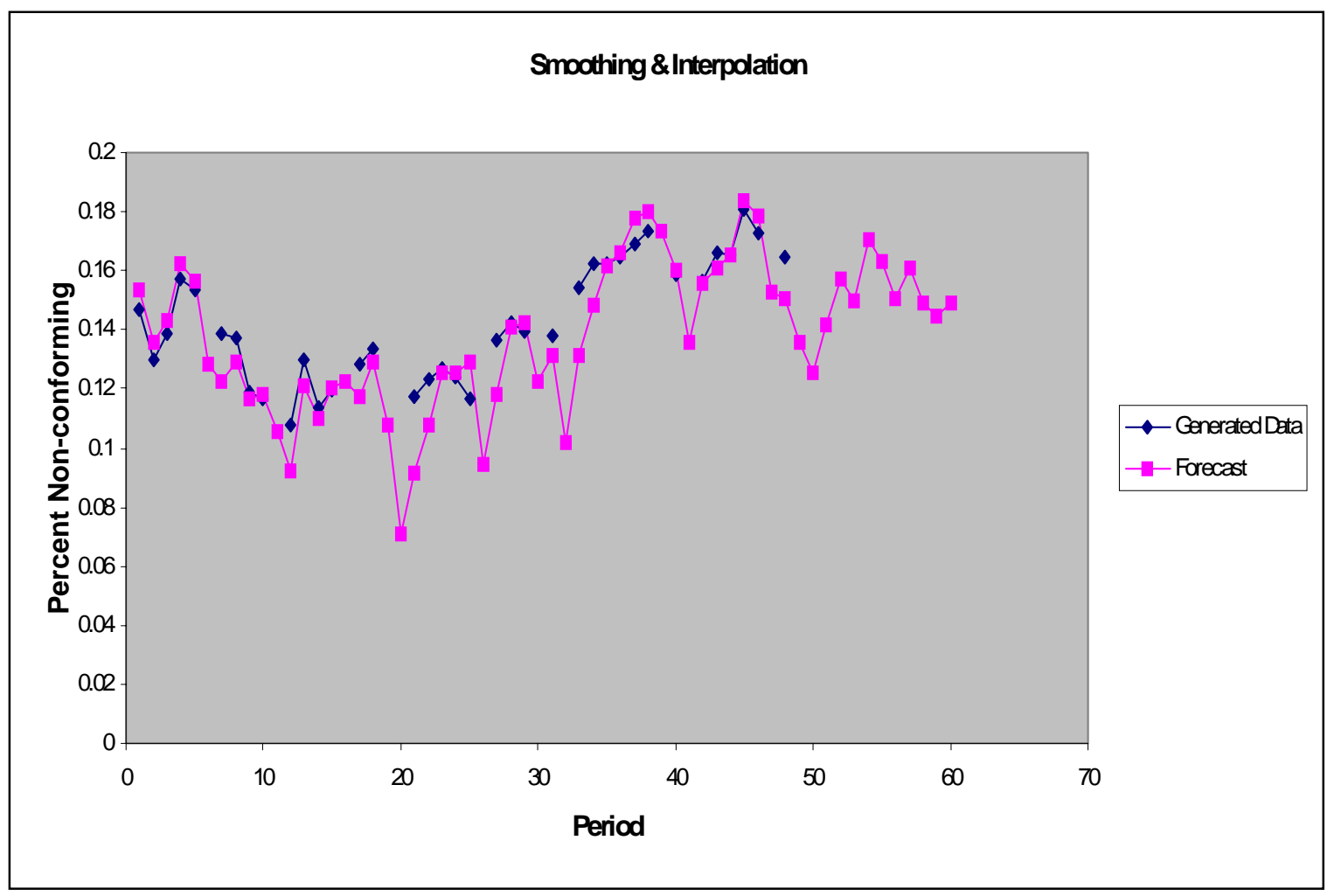

Figure 4.3.2(b) Generated vs. Forecasted values, ARMA(1,1) process, 11 observations missing 


\begin{tabular}{|c|c|c|c|c|c|c|c|}
\hline Period & 13 obs. Missing & Estimate & Forecast & Period & 13 obs. Missing & Estimate & Forecast \\
\hline 1 & 0.1465 & 0.148913 & 0.157766 & 31 & 0.138325026 & 0.126992 & 0.136631 \\
\hline 2 & 0.130053586 & 0.131088 & 0.139826 & 32 & & 0.102395 & 0.108961 \\
\hline 3 & 0.138678662 & 0.138381 & 0.147153 & 33 & 0.15460471 & 0.126365 & 0.136069 \\
\hline 4 & 0.157103537 & 0.158063 & 0.166728 & 34 & & 0.08855 & 0.095122 \\
\hline 5 & 0.153766381 & 0.151151 & 0.160106 & 35 & 0.162554885 & 0.129892 & 0.140093 \\
\hline 6 & & 0.12782 & 0.1321 & 36 & 0.164281013 & 0.140886 & 0.153687 \\
\hline 7 & 0.138591559 & 0.120463 & 0.126758 & 37 & 0.169349983 & 0.161298 & 0.175242 \\
\hline 8 & 0.137380734 & 0.125169 & 0.132821 & 38 & 0.173767257 & 0.16533 & 0.180211 \\
\hline 9 & 0.118681376 & 0.112171 & 0.120546 & 39 & & 0.144685 & 0.15422 \\
\hline 10 & 0.11673186 & 0.113595 & 0.122319 & 40 & 0.158672836 & 0.146437 & 0.157332 \\
\hline 11 & & 0.105432 & 0.109881 & 41 & & 0.124612 & 0.132189 \\
\hline 12 & 0.107904413 & 0.08996 & 0.096402 & 42 & 0.156703571 & 0.150334 & 0.160146 \\
\hline 13 & 0.129791784 & 0.117269 & 0.125103 & 43 & 0.166318687 & 0.15355 & 0.16478 \\
\hline 14 & 0.113370163 & 0.105398 & 0.114118 & 44 & 0.165603794 & 0.157776 & 0.170498 \\
\hline 15 & 0.119406005 & 0.11495 & 0.124165 & 45 & 0.180469625 & 0.175411 & 0.188695 \\
\hline 16 & & 0.120469 & 0.126509 & 46 & 0.172775322 & 0.166445 & 0.180627 \\
\hline 17 & 0.128191506 & 0.114073 & 0.121681 & 47 & & 0.172603 & 0.182248 \\
\hline 18 & 0.133870786 & 0.123948 & 0.132964 & 48 & & 0.123025 & 0.129353 \\
\hline 19 & & 0.105905 & 0.111886 & 49 & & & 0.099181 \\
\hline 20 & & 0.071059 & 0.075174 & 50 & & & 0.086223 \\
\hline 21 & 0.117405078 & 0.088133 & 0.095501 & 51 & & & 0.078405 \\
\hline 22 & 0.122978165 & 0.102304 & 0.111969 & 52 & & & 0.095425 \\
\hline 23 & 0.126881878 & 0.11885 & 0.129654 & 53 & & & 0.082307 \\
\hline 24 & 0.124074717 & 0.117707 & 0.129219 & 54 & & & 0.10434 \\
\hline 25 & & 0.117401 & 0.125281 & 55 & & & 0.089884 \\
\hline 26 & 0.127978247 & 0.108881 & 0.118883 & 56 & & & 0.074299 \\
\hline 27 & 0.136740953 & 0.124246 & 0.135637 & 57 & & & 0.07992 \\
\hline 28 & 0.14215751 & 0.140509 & 0.152134 & 58 & & & 0.05502 \\
\hline 29 & 0.139708315 & 0.140247 & 0.151811 & 59 & & & 0.071473 \\
\hline 30 & & 0.126227 & 0.134259 & 60 & & & 0.0526 \\
\hline
\end{tabular}

Table 4.3.3 Forecasting fraction non-conforming for data generated using ARMA(1,1) process (13 observations missing) 


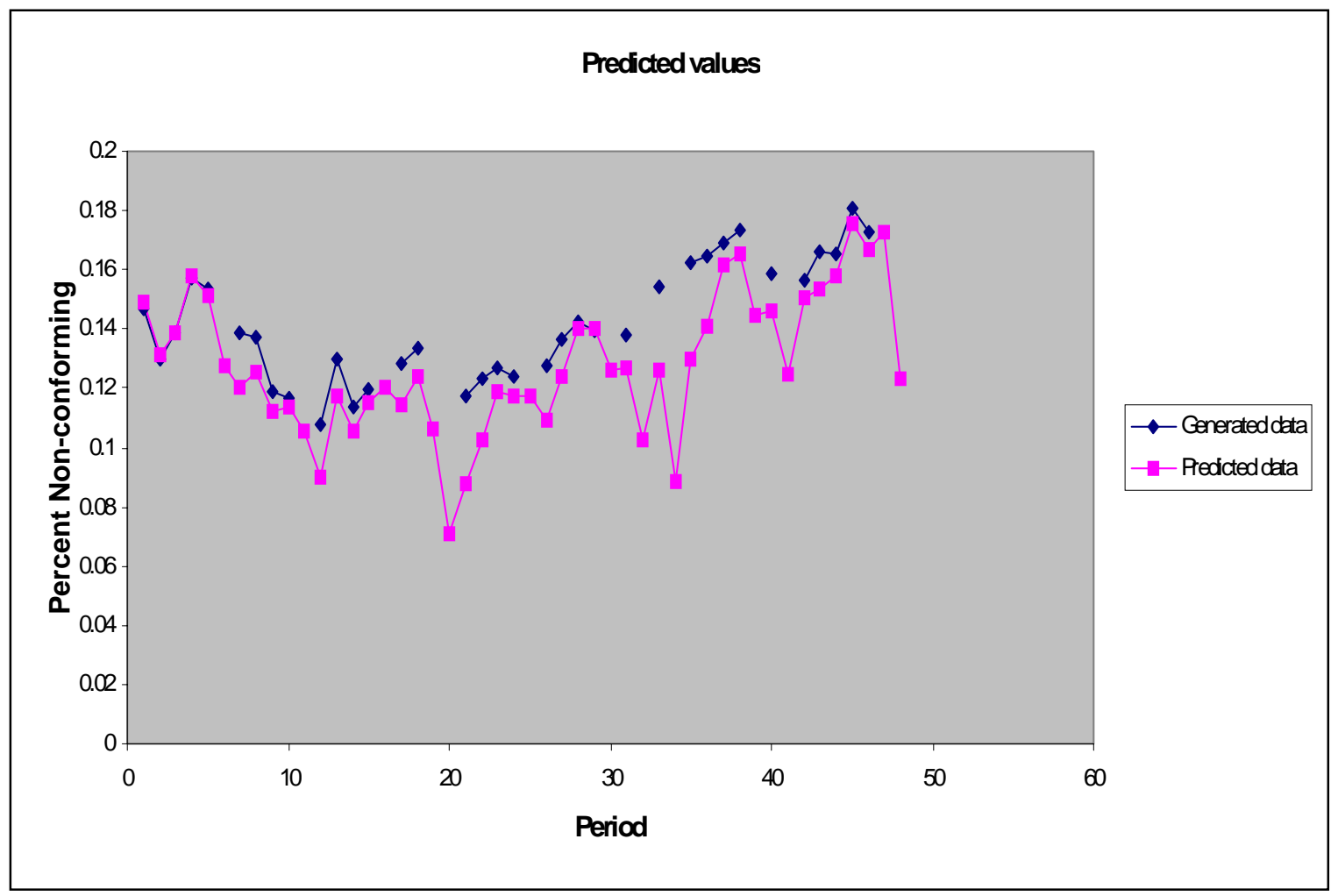

Figure 4.3.3(a) Generated vs. Predicted values, ARMA(1,1) process, 13 observations missing

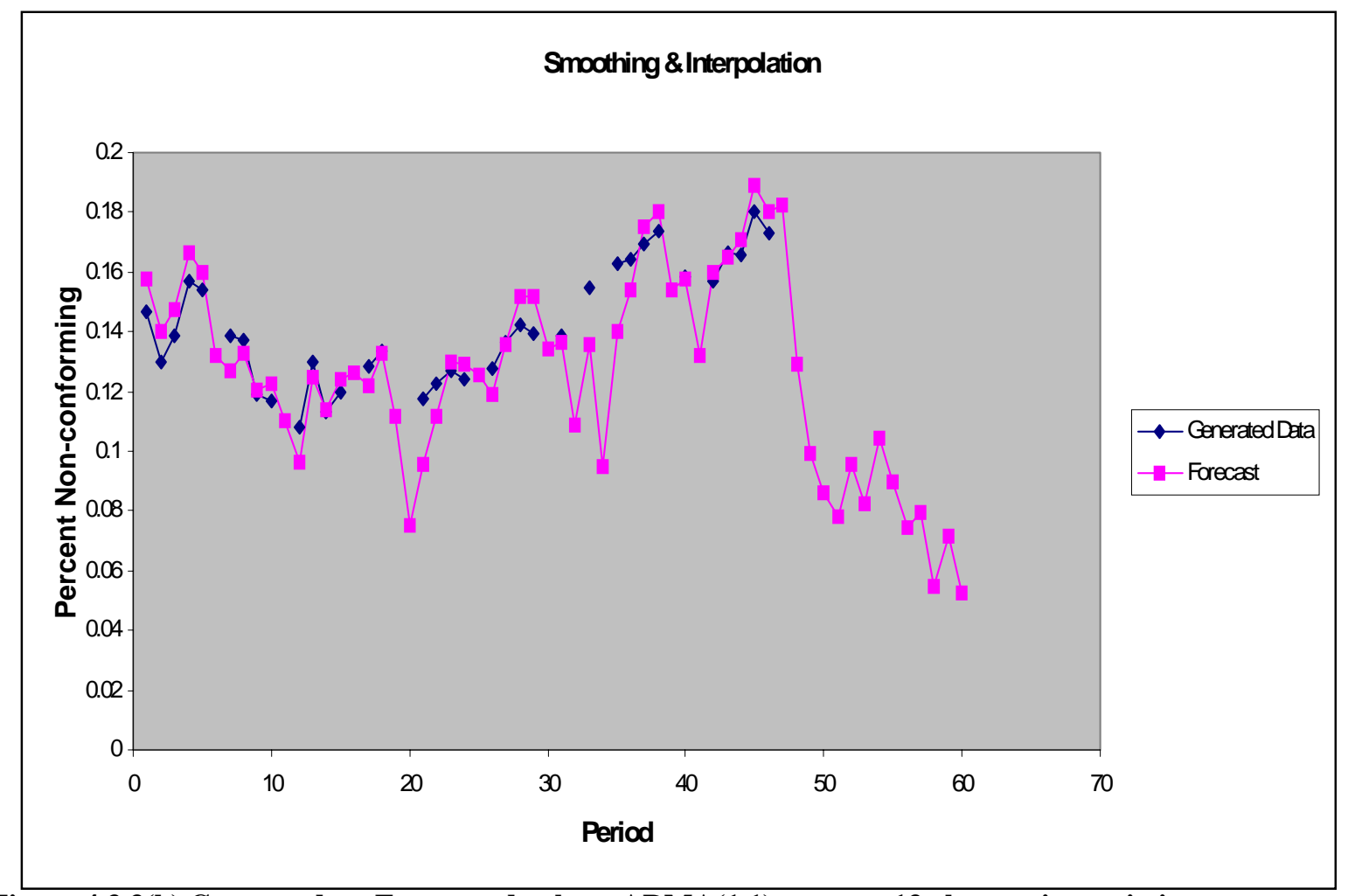

Figure 4.3.3(b) Generated vs. Forecasted values, ARMA(1,1) process, 13 observations missing 


\begin{tabular}{|c|c|c|c|c|c|c|c|}
\hline Period & 18 obs. Missing & Estimate & Forecast & Period & 18 obs. Missing & Estimate & Forecast \\
\hline 1 & 0.1465 & 0.148913 & 0.147766 & 31 & 0.138325026 & 0.122751 & 0.139308 \\
\hline 2 & 0.130053586 & 0.131088 & 0.146826 & 32 & & 0.09291 & 0.107023 \\
\hline 3 & & 0.112233 & 0.12266 & 33 & 0.15460471 & 0.124695 & 0.142131 \\
\hline 4 & 0.157103537 & 0.136899 & 0.149571 & 34 & 0.162430559 & 0.142296 & 0.16197 \\
\hline 5 & 0.153766381 & 0.140084 & 0.154277 & 35 & 0.162554885 & 0.150348 & 0.171379 \\
\hline 6 & & 0.104523 & 0.115149 & 36 & & 0.119525 & 0.135473 \\
\hline 7 & 0.138591559 & 0.114237 & 0.127569 & 37 & 0.169349983 & 0.147506 & 0.165882 \\
\hline 8 & 0.137380734 & 0.121447 & 0.13655 & 38 & 0.173767257 & 0.153985 & 0.174559 \\
\hline 9 & 0.118681376 & 0.1101 & 0.126157 & 39 & & 0.125542 & 0.141629 \\
\hline 10 & & 0.096718 & 0.108542 & 40 & 0.158672836 & 0.144779 & 0.16241 \\
\hline 11 & & 0.061353 & 0.071001 & 41 & & 0.130784 & 0.145823 \\
\hline 12 & 0.107904413 & 0.073166 & 0.086674 & 42 & & 0.107998 & 0.121616 \\
\hline 13 & 0.129791784 & 0.112027 & 0.127509 & 43 & 0.166318687 & 0.140805 & 0.157258 \\
\hline 14 & 0.113370163 & 0.102486 & 0.119178 & 44 & 0.165603794 & 0.151588 & 0.17071 \\
\hline 15 & 0.119406005 & 0.113532 & 0.131056 & 45 & & 0.137346 & 0.153669 \\
\hline 16 & & 0.137517 & 0.152398 & 46 & 0.172775322 & 0.151856 & 0.170503 \\
\hline 17 & & 0.104615 & 0.117688 & 47 & & 0.118637 & 0.134101 \\
\hline 18 & 0.133870786 & 0.116238 & 0.131812 & 48 & 0.164728272 & 0.145583 & 0.164694 \\
\hline 19 & & 0.096569 & 0.109948 & 49 & & & 0.157298 \\
\hline 20 & & 0.067176 & 0.07911 & 50 & & & 0.142531 \\
\hline 21 & 0.117405078 & 0.087543 & 0.102796 & 51 & & & 0.130571 \\
\hline 22 & 0.122978165 & 0.108145 & 0.125501 & 52 & & & 0.164312 \\
\hline 23 & 0.126881878 & 0.118083 & 0.136688 & 53 & & & 0.176466 \\
\hline 24 & & 0.087311 & 0.101895 & 54 & & & 0.15927 \\
\hline 25 & 0.116652999 & 0.10468 & 0.120595 & 55 & & & 0.183165 \\
\hline 26 & 0.127978247 & 0.109085 & 0.127099 & 56 & & & 0.169907 \\
\hline 27 & 0.136740953 & 0.123703 & 0.143166 & 57 & & & 0.166851 \\
\hline 28 & 0.14215751 & 0.144794 & 0.163884 & 58 & & & 0.1777 \\
\hline 29 & & 0.135974 & 0.152042 & 59 & & & 0.15768 \\
\hline 30 & & 0.105533 & 0.119881 & 60 & & & 0.170909 \\
\hline
\end{tabular}

Table 4.3.4 Forecasting fraction non-conforming for data generated using ARMA(1,1) process (18 observations missing) 


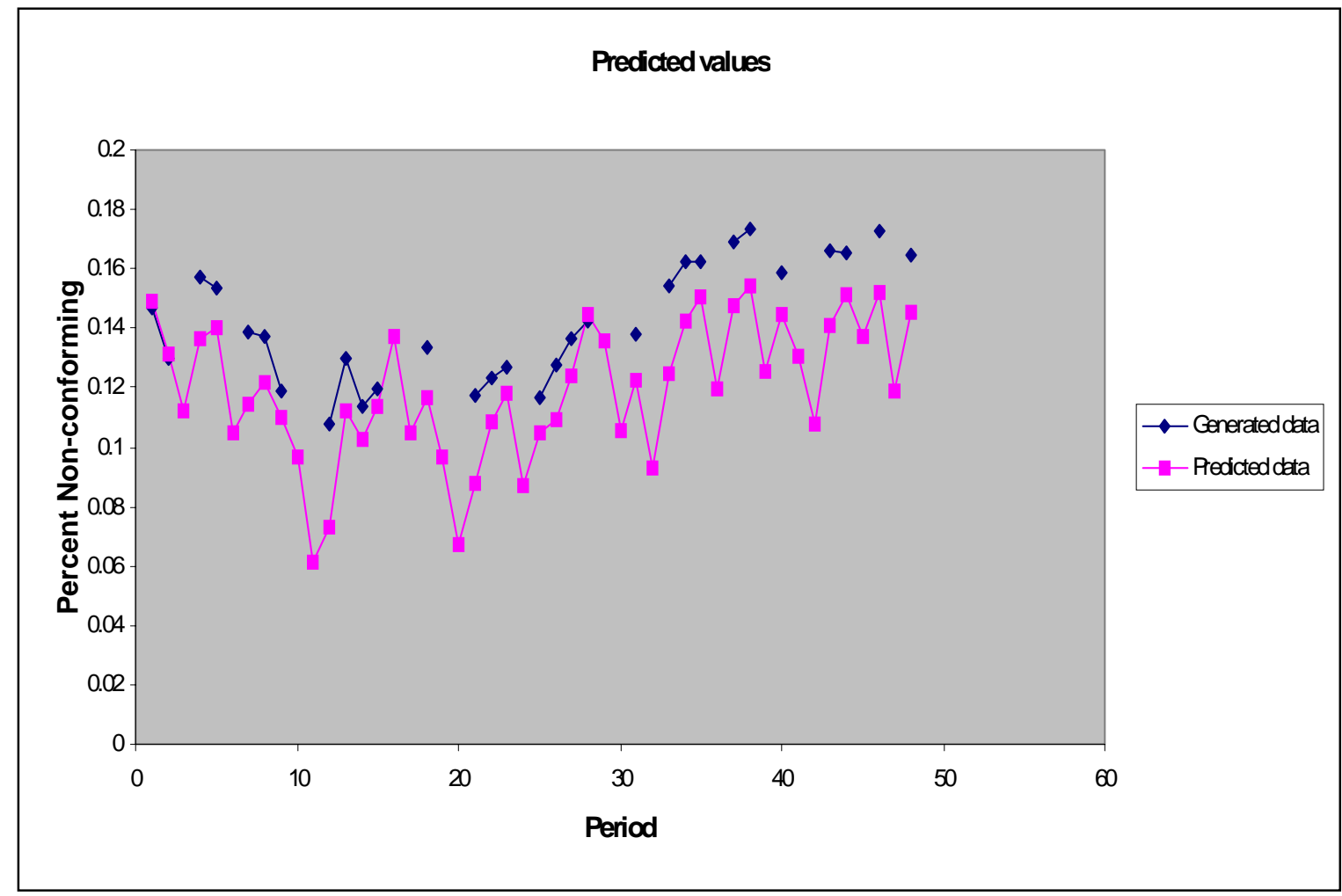

Figure 4.3.4(a) Generated vs. Predicted values, ARMA(1,1) process, 18 observations missing

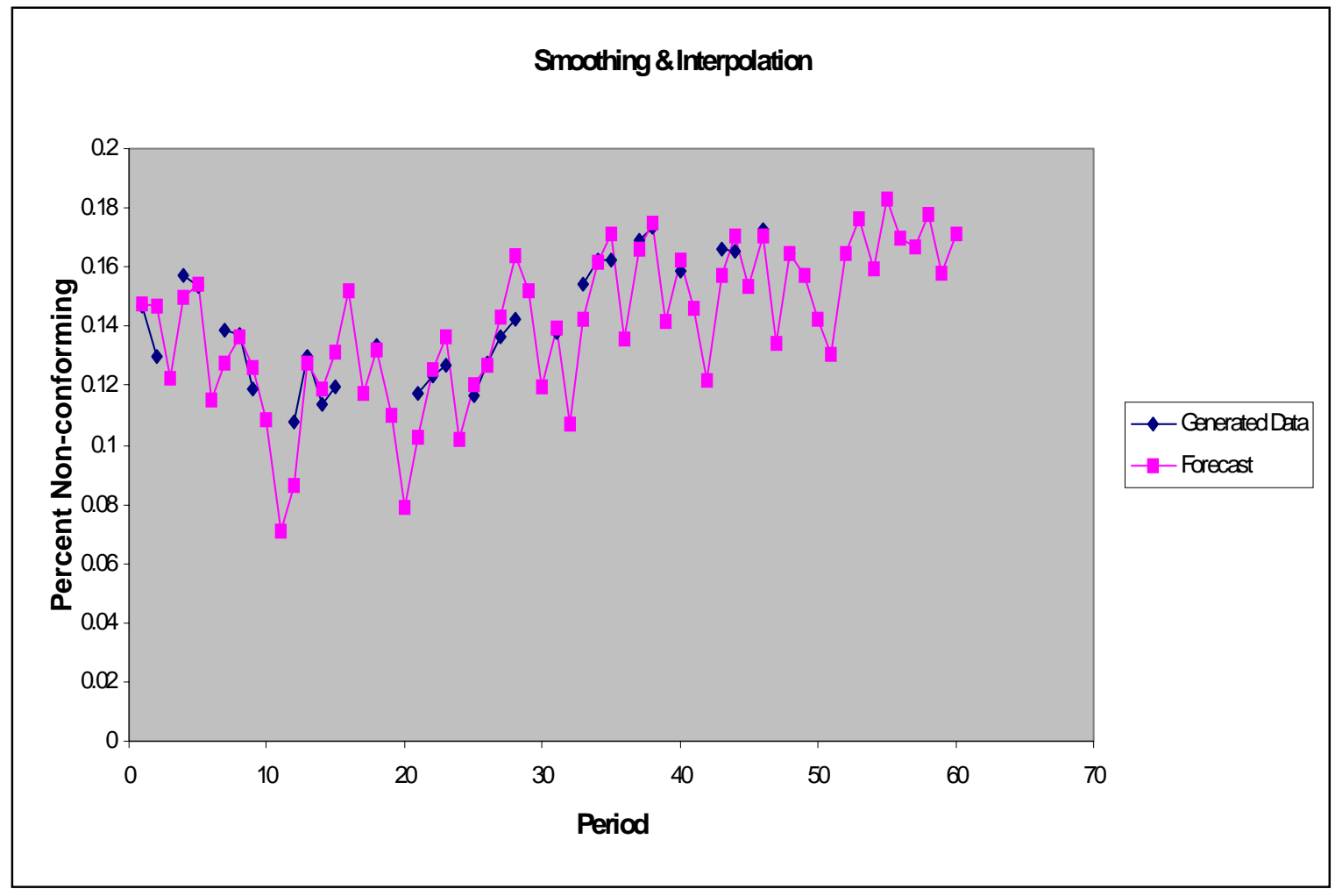

Figure 4.3.4(b) Generated vs. Predicted values, ARMA(1,1) process, 18 observations missing 


\begin{tabular}{|c|c|c|c|c|c|c|c|}
\hline Period & 11 obs. Missing & Estimate & Forecast & Period & 11 obs. Missing & Estimate & Forecast \\
\hline 1 & 0.066460039 & 0.070415 & 0.069831 & 31 & 0.057862093 & 0.055498 & 0.062382 \\
\hline 2 & 0.068406027 & 0.066673 & 0.066281 & 32 & 0.072386077 & 0.067588 & 0.075004 \\
\hline 3 & 0.069022682 & 0.071403 & 0.070747 & 33 & 0.066056977 & 0.065613 & 0.073079 \\
\hline 4 & 0.060061276 & 0.057793 & 0.057389 & 34 & & 0.044668 & 0.049475 \\
\hline 5 & 0.059611554 & 0.061685 & 0.061051 & 35 & 0.070092187 & 0.06055 & 0.066417 \\
\hline 6 & & 0.048523 & 0.045204 & 36 & 0.083032668 & 0.080482 & 0.086711 \\
\hline 7 & 0.061069648 & 0.047798 & 0.050953 & 37 & 0.077154064 & 0.071375 & 0.078246 \\
\hline 8 & 0.063367129 & 0.054482 & 0.060125 & 38 & 0.045250608 & 0.049604 & 0.055991 \\
\hline 9 & 0.077372599 & 0.068289 & 0.074942 & 39 & 0.072075606 & 0.06868 & 0.075444 \\
\hline 10 & & 0.040029 & 0.043566 & 40 & & 0.04246 & 0.046803 \\
\hline 11 & 0.078609831 & 0.061984 & 0.067368 & 41 & 0.032493901 & 0.036643 & 0.040525 \\
\hline 12 & 0.095618202 & 0.088144 & 0.094359 & 42 & & 0.024588 & 0.028062 \\
\hline 13 & 0.086649656 & 0.077237 & 0.084498 & 43 & 0.040396009 & 0.030819 & 0.035358 \\
\hline 14 & & 0.055647 & 0.06016 & 44 & & 0.027134 & 0.031244 \\
\hline 15 & 0.086791981 & 0.071725 & 0.077912 & 45 & 0.034510139 & 0.02885 & 0.033589 \\
\hline 16 & & 0.035754 & 0.039407 & 46 & 0.042137827 & 0.033651 & 0.039593 \\
\hline 17 & 0.077484967 & 0.058338 & 0.064118 & 47 & 0.055724682 & 0.052387 & 0.0587 \\
\hline 18 & 0.064368993 & 0.058361 & 0.064993 & 48 & 0.045733141 & 0.053361 & 0.058827 \\
\hline 19 & & 0.046773 & 0.05118 & 49 & & & 0.042156 \\
\hline 20 & 0.059921581 & \begin{tabular}{|l|}
0.049146 \\
\end{tabular} & 0.054751 & 50 & & & 0.023817 \\
\hline 21 & 0.05451498 & 0.05132 & 0.057279 & 51 & & & 0.0382 \\
\hline 22 & 0.055040064 & 0.048739 & 0.055593 & 52 & & & 0.011286 \\
\hline 23 & 0.061028263 & 0.063222 & 0.069832 & 53 & & & 0.034813 \\
\hline 24 & & 0.080415 & 0.086186 & 54 & & & 0.025971 \\
\hline 25 & 0.073778102 & 0.066507 & 0.073086 & 55 & & & 0.025998 \\
\hline 26 & 0.068166136 & 0.06315 & 0.070442 & 56 & & & 0.021755 \\
\hline 27 & 0.068164771 & 0.07102 & 0.077994 & 57 & & & 0.024308 \\
\hline 28 & & 0.04252 & 0.04688 & 58 & & & 0.017798 \\
\hline 29 & 0.072138127 & 0.060195 & 0.065882 & 59 & & & 0.032348 \\
\hline 30 & 0.06517116 & 0.05742 & 0.063968 & 60 & & & 0.040951 \\
\hline
\end{tabular}

Table 4.4.1 Forecasting fraction non-conforming for data generated using ARMA(1,2) process (11 observations missing) 


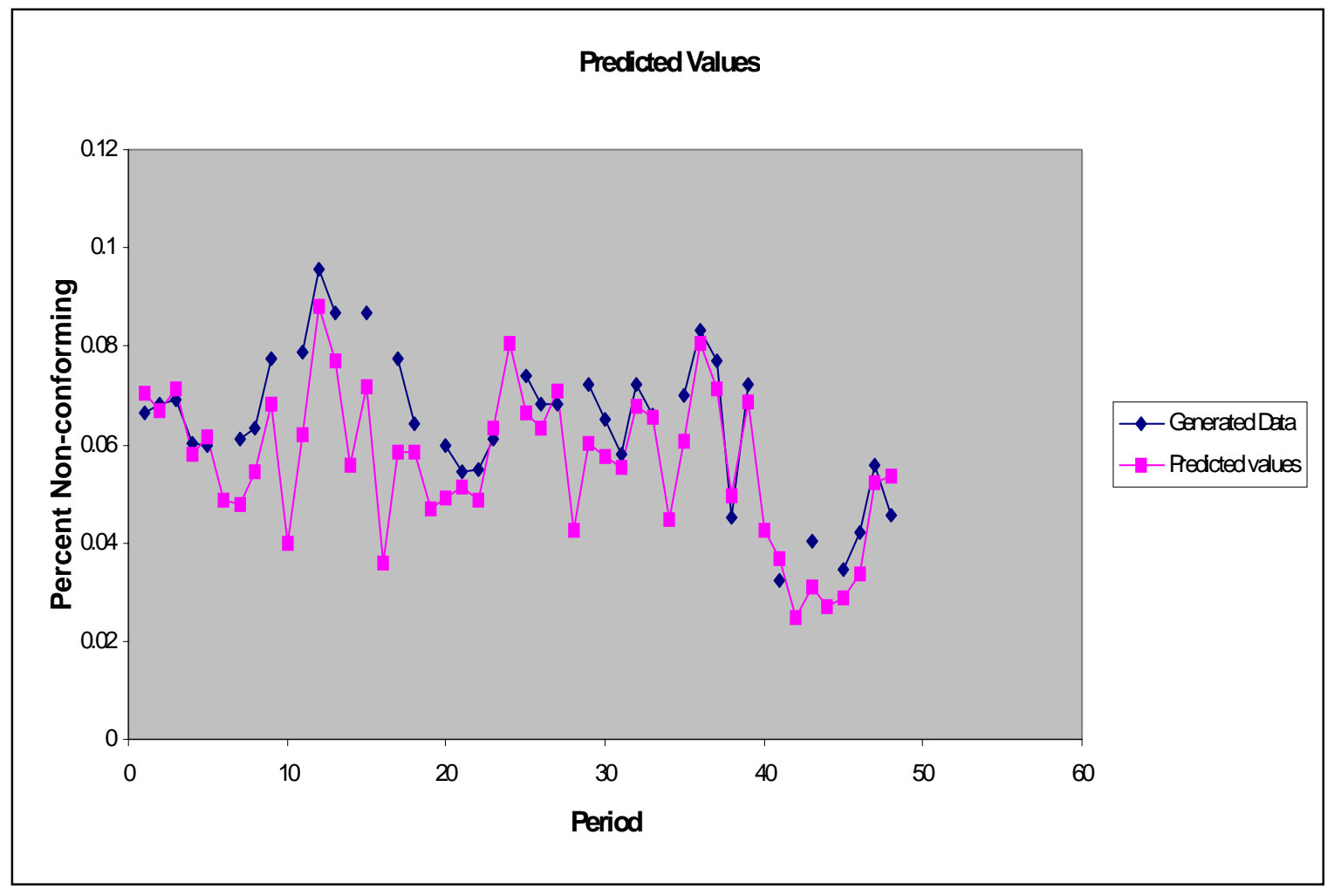

Figure 4.4.1(a) Generated vs. Predicted values, ARMA(1,2) process, 11 observations missing

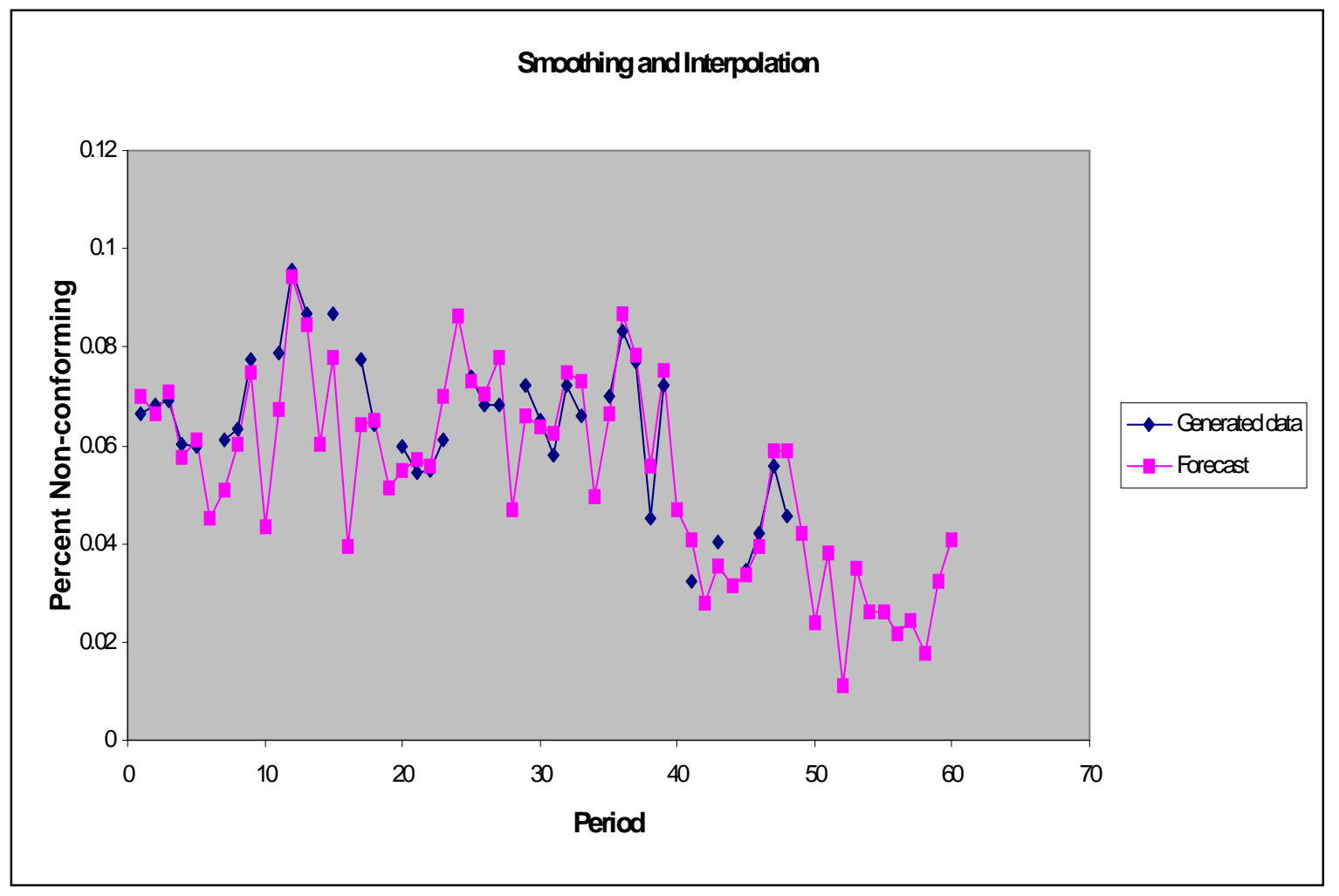

Figure 4.4.1(b) Generated vs. Forecasted values, ARMA(1,2) process, 11 observations missing 


\begin{tabular}{|c|c|c|c|c|c|c|c|}
\hline Period & 15 obs. Missing & Estimate & Forecast & Period & 15 obs. Missing & Estimate & Forecast \\
\hline 1 & 0.066460039 & 0.070415 & 0.069831 & 31 & 0.057862093 & 0.05417 & 0.062805 \\
\hline 2 & 0.068406027 & 0.066673 & 0.066281 & 32 & & 0.049323 & 0.056168 \\
\hline 3 & 0.069022682 & 0.071403 & 0.070747 & 33 & 0.066056977 & 0.055578 & 0.063587 \\
\hline 4 & 0.060061276 & 0.057793 & 0.057389 & 34 & & 0.029816 & 0.035873 \\
\hline 5 & 0.059611554 & 0.061685 & 0.061051 & 35 & 0.070092187 & 0.056641 & 0.064193 \\
\hline 6 & & 0.048523 & 0.053204 & 36 & 0.083032668 & 0.078697 & 0.086864 \\
\hline 7 & 0.061069648 & 0.047798 & 0.053953 & 37 & & 0.053361 & 0.060432 \\
\hline 8 & 0.063367129 & 0.054482 & 0.061625 & 38 & 0.045250608 & 0.041975 & 0.049671 \\
\hline 9 & 0.077372599 & 0.068289 & 0.076442 & 39 & 0.072075606 & 0.067336 & 0.075558 \\
\hline 10 & & 0.040029 & 0.045066 & 40 & & 0.032859 & 0.039048 \\
\hline 11 & 0.078609831 & 0.061984 & 0.068868 & 41 & 0.032493901 & 0.035782 & 0.041605 \\
\hline 12 & 0.095618202 & 0.088144 & 0.095859 & 42 & & 0.02338 & 0.029035 \\
\hline 13 & 0.086649656 & 0.077237 & 0.085998 & 43 & 0.040396009 & 0.030999 & 0.037698 \\
\hline 14 & & 0.055647 & 0.06166 & 44 & & 0.024014 & 0.030178 \\
\hline 15 & 0.086791981 & 0.071725 & 0.079412 & 45 & & 0.024481 & 0.030658 \\
\hline 16 & & 0.035754 & 0.040907 & 46 & 0.042137827 & 0.0316 & 0.039272 \\
\hline 17 & 0.077484967 & 0.058338 & 0.065618 & 47 & 0.055724682 & 0.051837 & 0.05994 \\
\hline 18 & 0.064368993 & 0.058361 & 0.066493 & 48 & 0.045733141 & 0.052922 & 0.060227 \\
\hline 19 & & 0.046773 & 0.05268 & 49 & & & 0.035898 \\
\hline 20 & 0.059921581 & 0.049146 & 0.056251 & 50 & & & 0.025079 \\
\hline 21 & 0.05451498 & 0.05132 & 0.058779 & 51 & & & 0.044784 \\
\hline 22 & 0.055040064 & 0.048739 & 0.057093 & 52 & & & 0.009898 \\
\hline 23 & 0.061028263 & 0.063222 & 0.071332 & 53 & & & 0.037418 \\
\hline 24 & & 0.080415 & 0.087686 & 54 & & & 0.02717 \\
\hline 25 & 0.073778102 & 0.066507 & 0.074586 & 55 & & & 0.027005 \\
\hline 26 & & 0.04608 & 0.052062 & 56 & & & 0.021723 \\
\hline 27 & 0.068164771 & 0.059897 & 0.066798 & 57 & & & 0.027532 \\
\hline 28 & & 0.024057 & 0.029149 & 58 & & & 0.020757 \\
\hline 29 & 0.072138127 & 0.055574 & 0.062506 & 59 & & & 0.036306 \\
\hline 30 & 0.06517116 & 0.054556 & 0.062667 & 60 & & & 0.044581 \\
\hline
\end{tabular}

Table 4.4.2 Forecasting fraction non-conforming for data generated using ARMA(1,2) process (15 observations missing) 


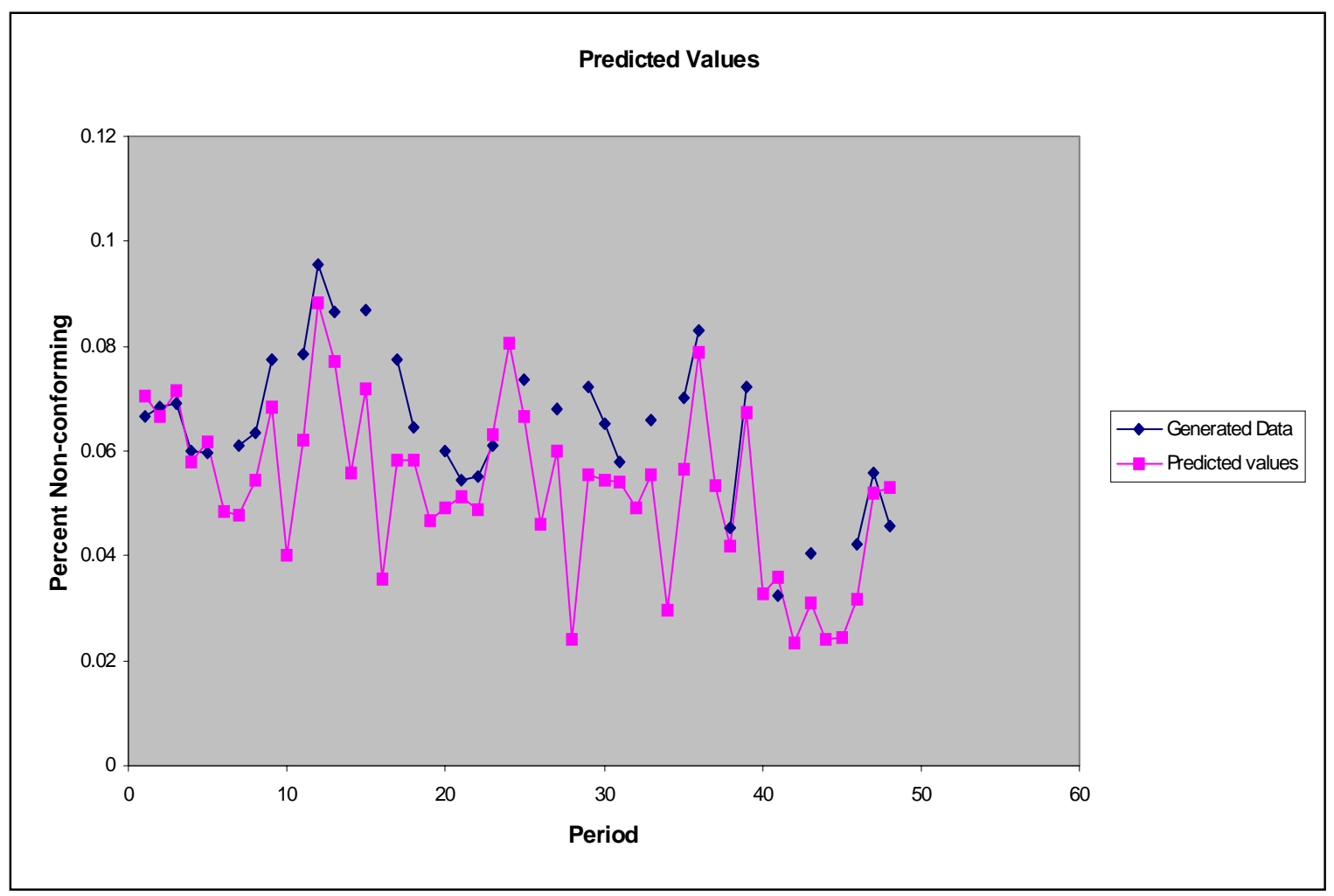

Figure 4.4.2(a) Generated vs. Predicted values, ARMA(1,2) process, 15 observations missing

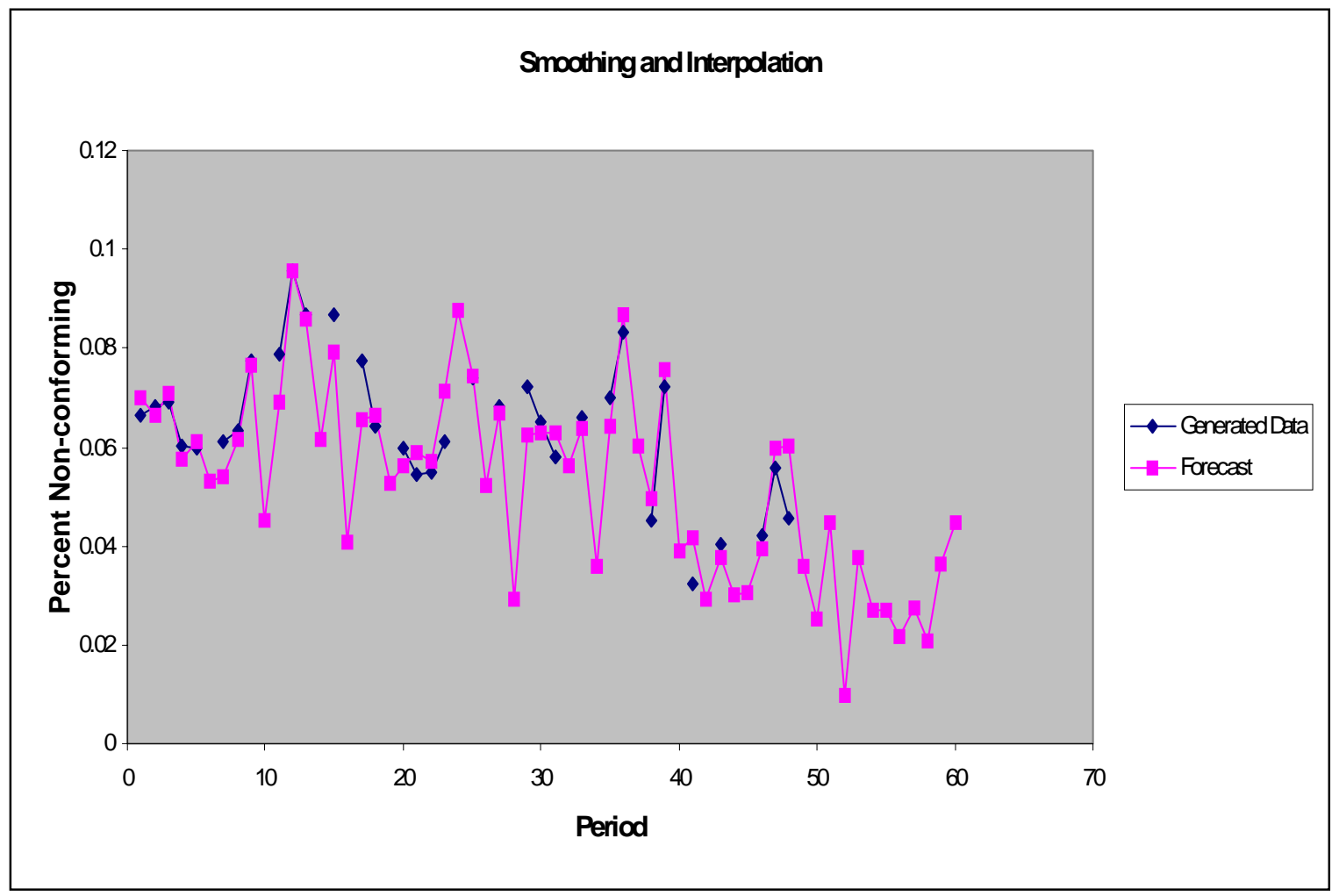

Figure 4.4.2(b) Generated vs. Predicted values, ARMA(1,2) process, 15 observations missing 


\begin{tabular}{|c|c|c|c|c|c|c|c|}
\hline Period & 17 obs. Missing & Estimate & Forecast & Period & 17 obs. Missing & Estimate & Forecast \\
\hline 1 & 0.066460039 & 0.070415 & 0.069831 & 31 & 0.057862093 & 0.046241 & 0.053812 \\
\hline 2 & 0.068406027 & 0.066673 & 0.071281 & 32 & & 0.034259 & 0.040758 \\
\hline 3 & 0.069022682 & 0.071403 & 0.070747 & 33 & 0.066056977 & 0.051579 & 0.059687 \\
\hline 4 & 0.060061276 & 0.057793 & 0.057389 & 34 & & 0.0243 & 0.030717 \\
\hline 5 & 0.059611554 & 0.061685 & 0.061051 & 35 & 0.070092187 & 0.055352 & 0.063408 \\
\hline 6 & & 0.048523 & & 36 & 0.083032668 & 0.078234 & 0.08697 \\
\hline 7 & 0.061069648 & 0.047798 & 0.053953 & 37 & & 0.049869 & 0.057332 \\
\hline 8 & 0.063367129 & 0.054482 & 0.061625 & 38 & 0.045250608 & 0.041598 & 0.049757 \\
\hline 9 & 0.077372599 & 0.068289 & 0.076442 & 39 & 0.072075606 & 0.068429 & 0.076994 \\
\hline 10 & & 0.040029 & 0.045066 & 40 & & 0.031387 & 0.037978 \\
\hline 11 & 0.078609831 & 0.061984 & 0.068868 & 41 & 0.032493901 & 0.036125 & 0.042312 \\
\hline 12 & 0.095618202 & 0.088144 & 0.095859 & 42 & & 0.015584 & 0.02134 \\
\hline 13 & 0.086649656 & 0.077237 & 0.085998 & 43 & 0.040396009 & 0.033125 & 0.039689 \\
\hline 14 & & 0.055647 & 0.06166 & 44 & & 0.018935 & 0.025186 \\
\hline 15 & 0.086791981 & 0.071725 & 0.079412 & 45 & & 0.024857 & 0.031356 \\
\hline 16 & & 0.035754 & 0.040907 & 46 & 0.042137827 & 0.031445 & 0.03946 \\
\hline 17 & 0.077484967 & 0.058338 & 0.065618 & 47 & 0.055724682 & 0.05215 & 0.060563 \\
\hline 18 & 0.064368993 & 0.058361 & 0.066493 & 48 & 0.045733141 & 0.053101 & 0.060695 \\
\hline 19 & & 0.046773 & 0.05268 & 49 & & & 0.031115 \\
\hline 20 & 0.059921581 & 0.049146 & 0.056251 & 50 & & & 0.026134 \\
\hline 21 & 0.05451498 & 0.05132 & 0.058779 & 51 & & & 0.048349 \\
\hline 22 & 0.055040064 & 0.048739 & 0.057093 & 52 & & & 0.01083 \\
\hline 23 & 0.061028263 & 0.063222 & 0.071332 & 53 & & & 0.039855 \\
\hline 24 & & 0.080415 & 0.087686 & 54 & & & 0.021604 \\
\hline 25 & & 0.048313 & 0.054268 & 55 & & & 0.036329 \\
\hline 26 & & 0.024625 & 0.029417 & 56 & & & 0.023812 \\
\hline 27 & 0.068164771 & 0.05442 & 0.06074 & 57 & & & 0.032577 \\
\hline 28 & & 0.015144 & 0.020043 & 58 & & & 0.023601 \\
\hline 29 & 0.072138127 & 0.053407 & 0.060387 & 59 & & & 0.039914 \\
\hline 30 & & 0.027893 & 0.033816 & 60 & & & 0.048232 \\
\hline
\end{tabular}

Table 4.4.3 Forecasting fraction non-conforming for data generated using $\operatorname{ARMA}(1,2)$ process $(17$ observations missing) 


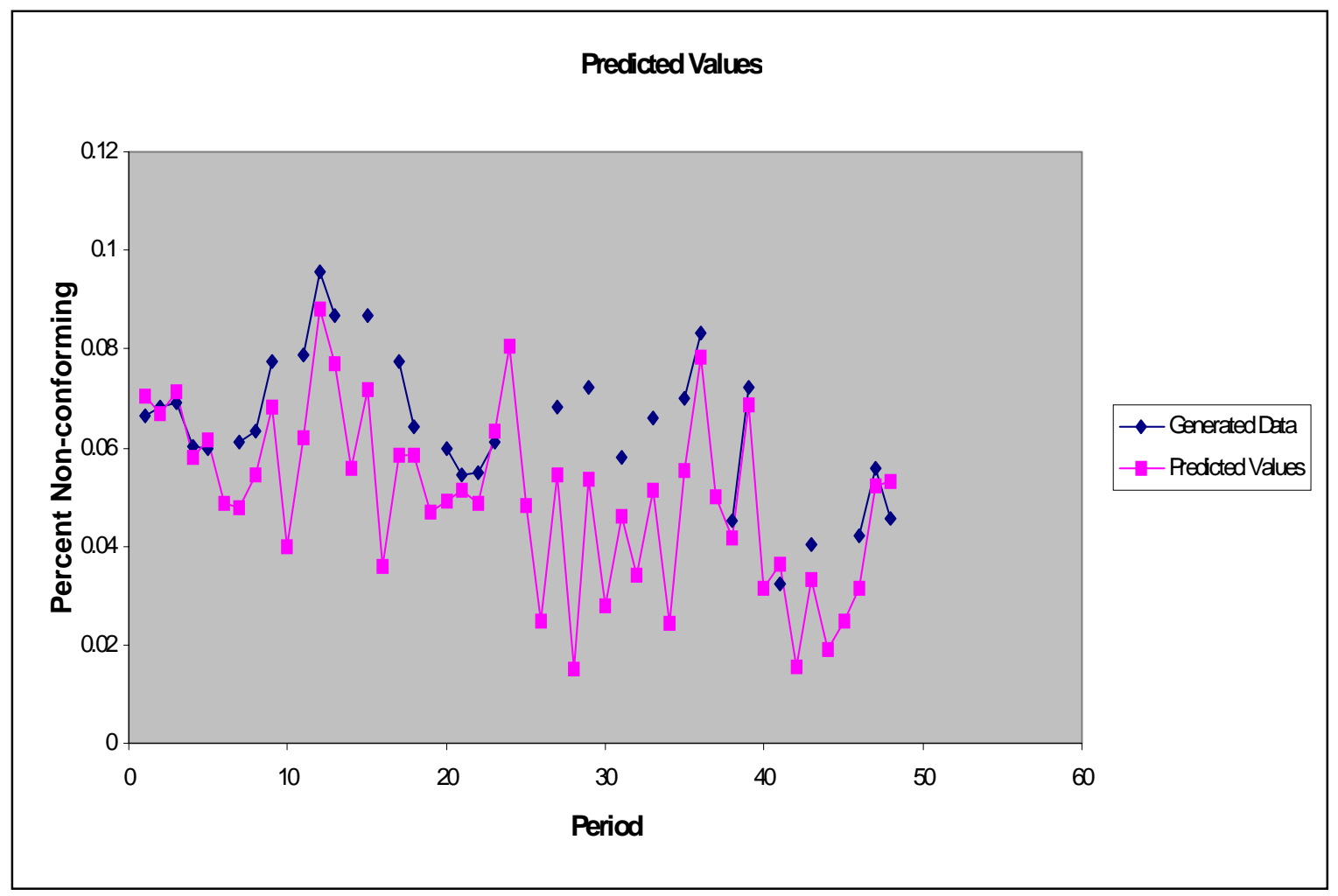

Figure 4.4.3(a) Generated vs. Predicted values, $\operatorname{ARMA(1,2)~process,~} 17$ observations missing

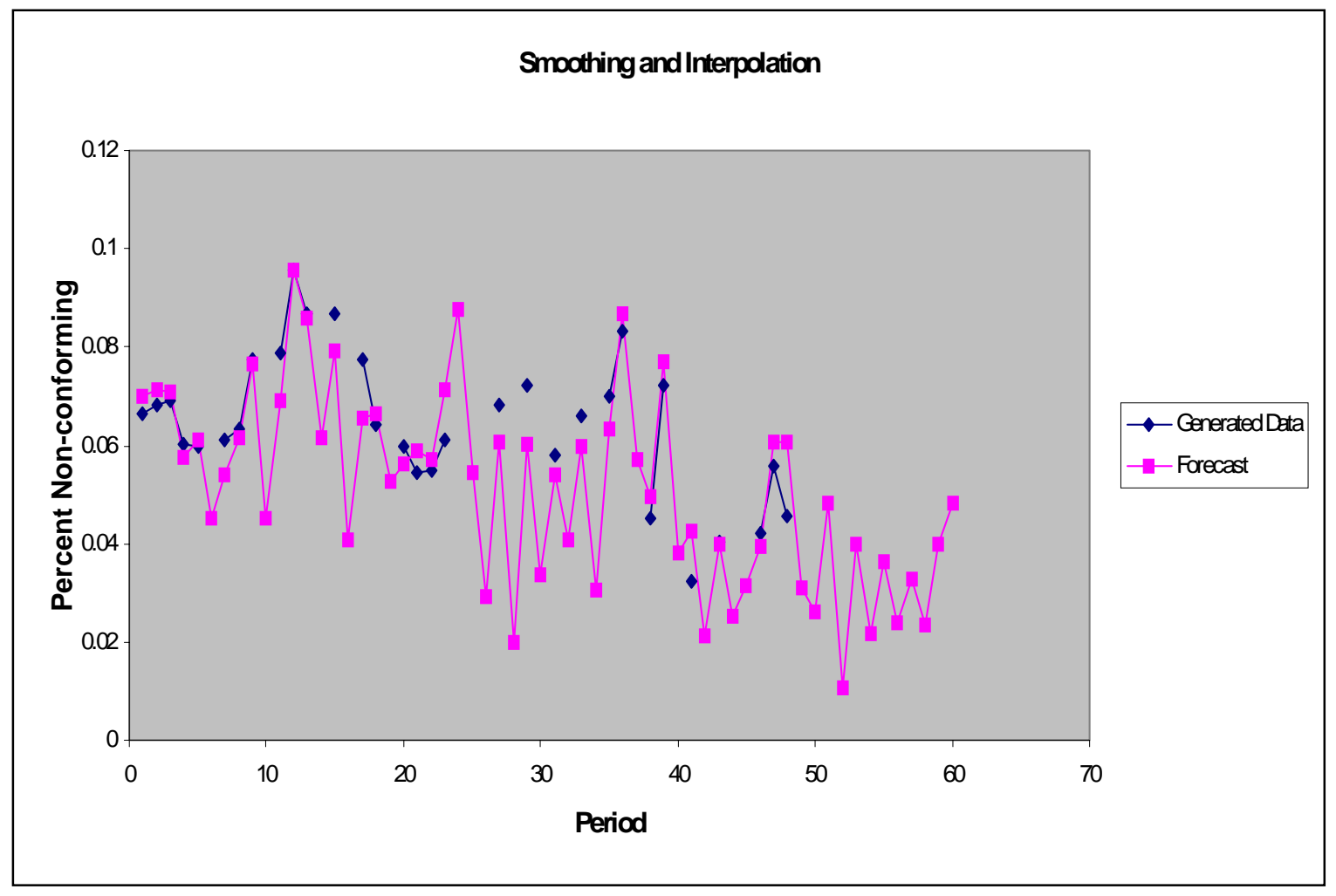

Figure 4.4.3(b) Generated vs. Forecasted values, ARMA(1,2) process, 17 observations missing 


\begin{tabular}{|c|c|c|c|c|c|c|c|}
\hline Period & 18 obs. Missing & Estimate & Forecast & Period & 18 obs. Missing & Estimate & Forecast \\
\hline 1 & 0.066460039 & 0.070415 & 0.069831 & 31 & 0.057862093 & 0.046241 & 0.052812 \\
\hline 2 & 0.068406027 & 0.066673 & 0.073281 & 32 & & 0.034259 & 0.039758 \\
\hline 3 & 0.069022682 & 0.071403 & 0.077747 & 33 & 0.066056977 & 0.051579 & 0.058687 \\
\hline 4 & 0.060061276 & 0.057793 & 0.064389 & 34 & & 0.0243 & 0.029717 \\
\hline 5 & 0.059611554 & 0.061685 & 0.068051 & 35 & 0.070092187 & 0.055352 & 0.062408 \\
\hline 6 & & 0.048523 & 0.052204 & 36 & 0.083032668 & 0.078234 & 0.08597 \\
\hline 7 & 0.061069648 & 0.047798 & 0.052953 & 37 & & 0.049869 & 0.056332 \\
\hline 8 & 0.063367129 & 0.054482 & 0.060625 & 38 & 0.045250608 & 0.041598 & 0.048757 \\
\hline 9 & 0.077372599 & 0.068289 & 0.075442 & 39 & 0.072075606 & 0.068429 & 0.075994 \\
\hline 10 & & 0.040029 & 0.044066 & 40 & & 0.031387 & 0.036978 \\
\hline 11 & 0.078609831 & 0.061984 & 0.067868 & 41 & 0.032493901 & 0.036125 & 0.041312 \\
\hline 12 & 0.095618202 & 0.088144 & 0.094859 & 42 & & 0.015584 & 0.02034 \\
\hline 13 & 0.086649656 & 0.077237 & 0.084998 & 43 & 0.040396009 & 0.033125 & 0.038689 \\
\hline 14 & & 0.055647 & 0.06066 & 44 & & 0.018935 & 0.024186 \\
\hline 15 & 0.086791981 & 0.071725 & 0.078412 & 45 & & 0.024857 & 0.030356 \\
\hline 16 & & 0.035754 & 0.039907 & 46 & 0.042137827 & 0.031445 & 0.03846 \\
\hline 17 & 0.077484967 & 0.058338 & 0.064618 & 47 & 0.055724682 & 0.05215 & 0.059563 \\
\hline 18 & 0.064368993 & 0.058361 & 0.065493 & 48 & & 0.067391 & 0.075101 \\
\hline 19 & & 0.046773 & 0.05168 & 49 & & & 0.043396 \\
\hline 20 & 0.059921581 & 0.049146 & 0.055251 & 50 & & & 0.039532 \\
\hline 21 & 0.05451498 & 0.05132 & 0.057779 & 51 & & & 0.062863 \\
\hline 22 & 0.055040064 & 0.048739 & 0.056093 & 52 & & & 0.026461 \\
\hline 23 & 0.061028263 & 0.063222 & 0.070332 & 53 & & & 0.056603 \\
\hline 24 & & 0.080415 & 0.086686 & 54 & & & 0.039468 \\
\hline 25 & & 0.048313 & 0.053268 & 55 & & & 0.05531 \\
\hline 26 & & 0.024625 & 0.028417 & 56 & & & 0.043909 \\
\hline 27 & 0.068164771 & 0.05442 & 0.05974 & 57 & & & 0.05379 \\
\hline 28 & & 0.015144 & 0.019043 & 58 & & & 0.045931 \\
\hline 29 & 0.072138127 & 0.053407 & 0.059387 & 59 & & & 0.06336 \\
\hline 30 & & 0.027893 & 0.032816 & 60 & & & 0.077707 \\
\hline
\end{tabular}

Table 4.4.4 Forecasting fraction non-conforming for data generated using ARMA(1,2) process (18 observations missing) 


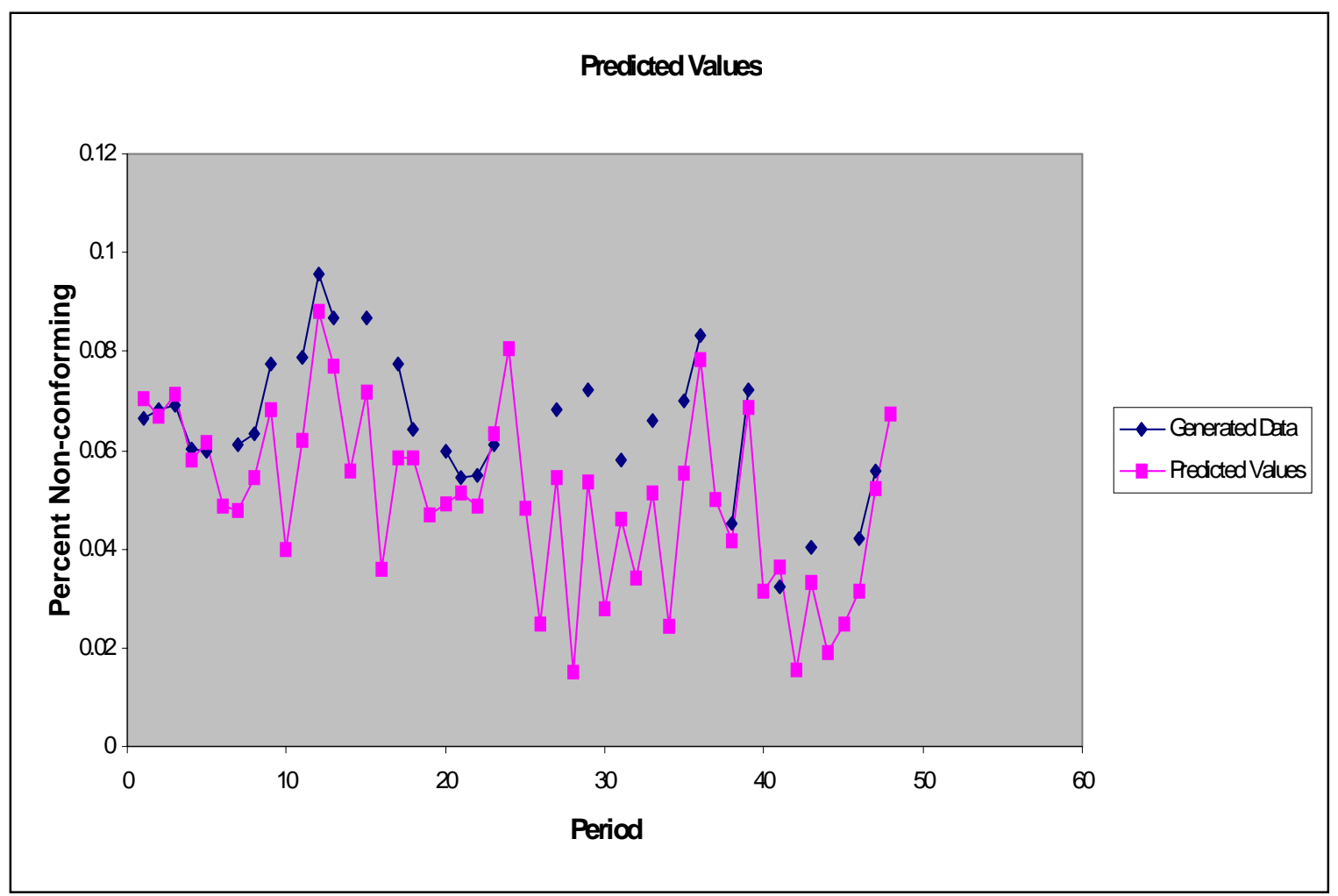

Figure 4.4.4(a) Generated vs. Predicted values, ARMA(1,2) process, 18 observations missing

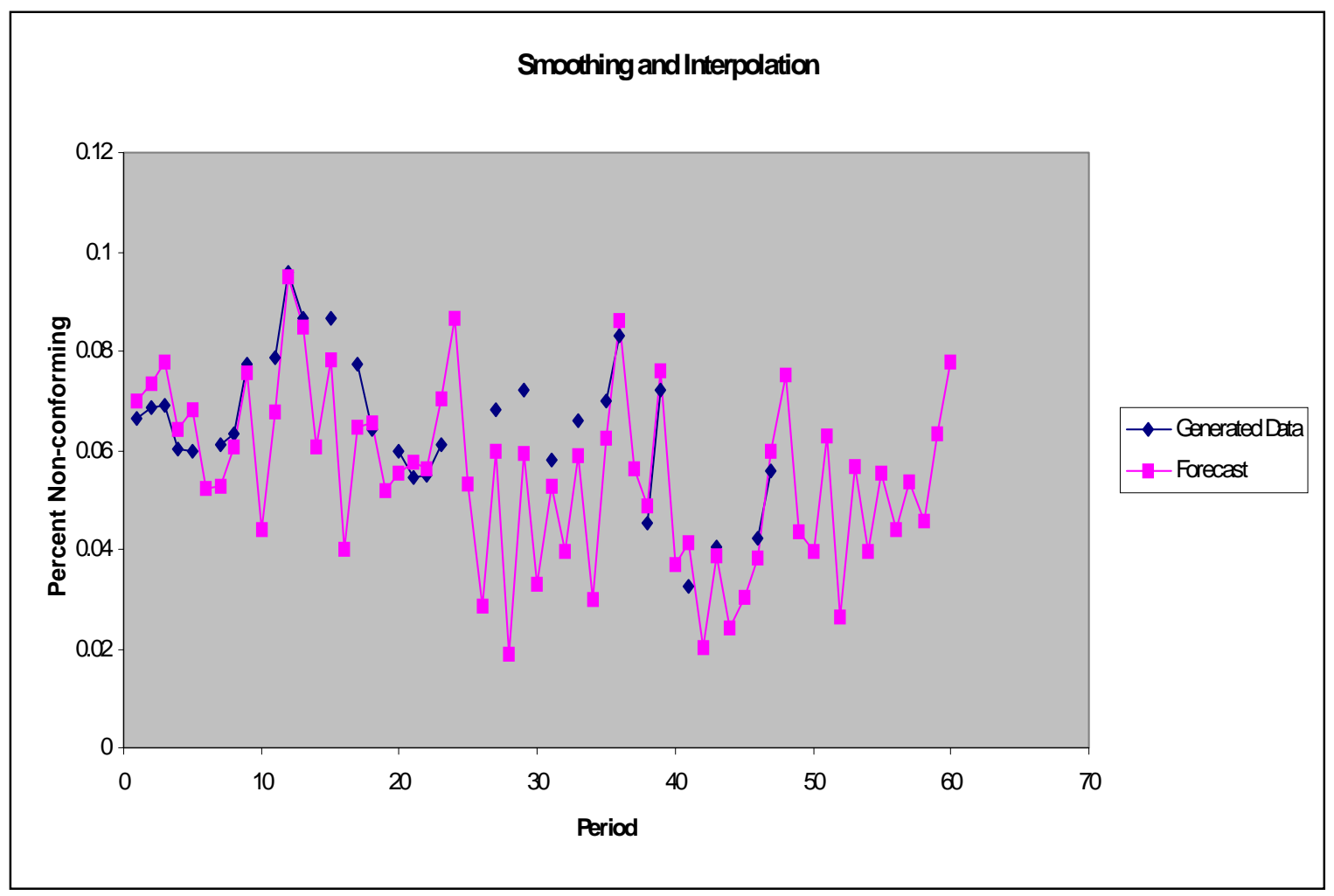

Figure 4.4.4(b) Forecasted vs. Predicted values, ARMA(1,2) process, 18 observations missing 


\begin{tabular}{|c|c|c|c|c|c|c|c|}
\hline Period & 11 obs. Missing & Estimate & Forecast & Period & 11 obs. Missing & Estimate & Forecast \\
\hline 1 & 0.101417725 & 0.091112 & 0.097957 & 31 & & 0.042256 & 0.049574 \\
\hline 2 & 0.104934133 & 0.109469 & 0.11581 & 32 & 0.052658789 & 0.038402 & 0.047305 \\
\hline 3 & & 0.016617 & 0.015801 & 33 & 0.070499177 & 0.059791 & 0.069884 \\
\hline 4 & 0.116023022 & 0.074885 & 0.078639 & 34 & 0.054464728 & 0.043861 & 0.055131 \\
\hline 5 & 0.080922007 & 0.071516 & 0.076315 & 35 & & 0.04343 & 0.051514 \\
\hline 6 & 0.099886633 & 0.093919 & 0.099381 & 36 & 0.0620485 & 0.056242 & 0.065149 \\
\hline 7 & 0.094682663 & 0.080712 & 0.087727 & 37 & 0.049443655 & 0.041285 & 0.051099 \\
\hline 8 & 0.07593737 & 0.070697 & 0.078294 & 38 & 0.062172423 & 0.0554 & 0.065967 \\
\hline 9 & 0.090285713 & 0.087723 & 0.095605 & 39 & & 0.017781 & 0.024943 \\
\hline 10 & & 0.030924 & 0.033445 & 40 & 0.042668614 & 0.030299 & 0.038835 \\
\hline 11 & 0.056614679 & 0.039837 & 0.044222 & 41 & 0.022828031 & 0.025768 & 0.033978 \\
\hline 12 & 0.067695322 & 0.060475 & 0.065662 & 42 & & 0.019874 & 0.026797 \\
\hline 13 & 0.044159983 & 0.033847 & 0.04018 & 43 & 0.023460506 & 0.019995 & 0.027409 \\
\hline 14 & & 0.03987 & 0.043707 & 44 & & 0.006119 & 0.012711 \\
\hline 15 & 0.037047144 & 0.014802 & 0.021794 & 45 & 0.022340746 & 0.01731 & 0.024462 \\
\hline 16 & 0.027258606 & 0.036536 & 0.042497 & 46 & 0.051637085 & 0.027037 & 0.036922 \\
\hline 17 & & 0.023539 & 0.027609 & 47 & 0.046641543 & 0.03957 & 0.050458 \\
\hline 18 & & 0.026992 & 0.031002 & 48 & 0.037209459 & 0.044729 & 0.054781 \\
\hline 19 & 0.037437339 & 0.022684 & 0.028333 & 49 & & & 0.026864 \\
\hline 20 & 0.033519587 & 0.019885 & 0.027049 & 50 & & & 0.033693 \\
\hline 21 & 0.047304722 & 0.03911 & 0.047185 & 51 & & & 0.009177 \\
\hline 22 & 0.032965677 & 0.023242 & 0.032696 & 52 & & & 0.031266 \\
\hline 23 & 0.038172224 & 0.039422 & 0.048737 & 53 & & & 0.031517 \\
\hline 24 & & 0.050894 & 0.058483 & 54 & & & 0.049418 \\
\hline 25 & 0.052574234 & 0.035393 & 0.044891 & 55 & & & 0.038668 \\
\hline 26 & 0.041473637 & 0.041936 & 0.051368 & 56 & & & 0.030719 \\
\hline 27 & 0.068509549 & 0.046154 & 0.05807 & 57 & & & 0.044714 \\
\hline 28 & 0.041733999 & 0.051267 & 0.062124 & 58 & & & 0.030008 \\
\hline 29 & 0.074192064 & 0.06937 & 0.08091 & 59 & & & 0.032679 \\
\hline 30 & 0.034715071 & 0.049303 & 0.058774 & 60 & & & 0.045356 \\
\hline
\end{tabular}

Table 4.6.2 Forecasting fraction non-conforming for data generated using ARMA(2,2) process (11 observations missing) 


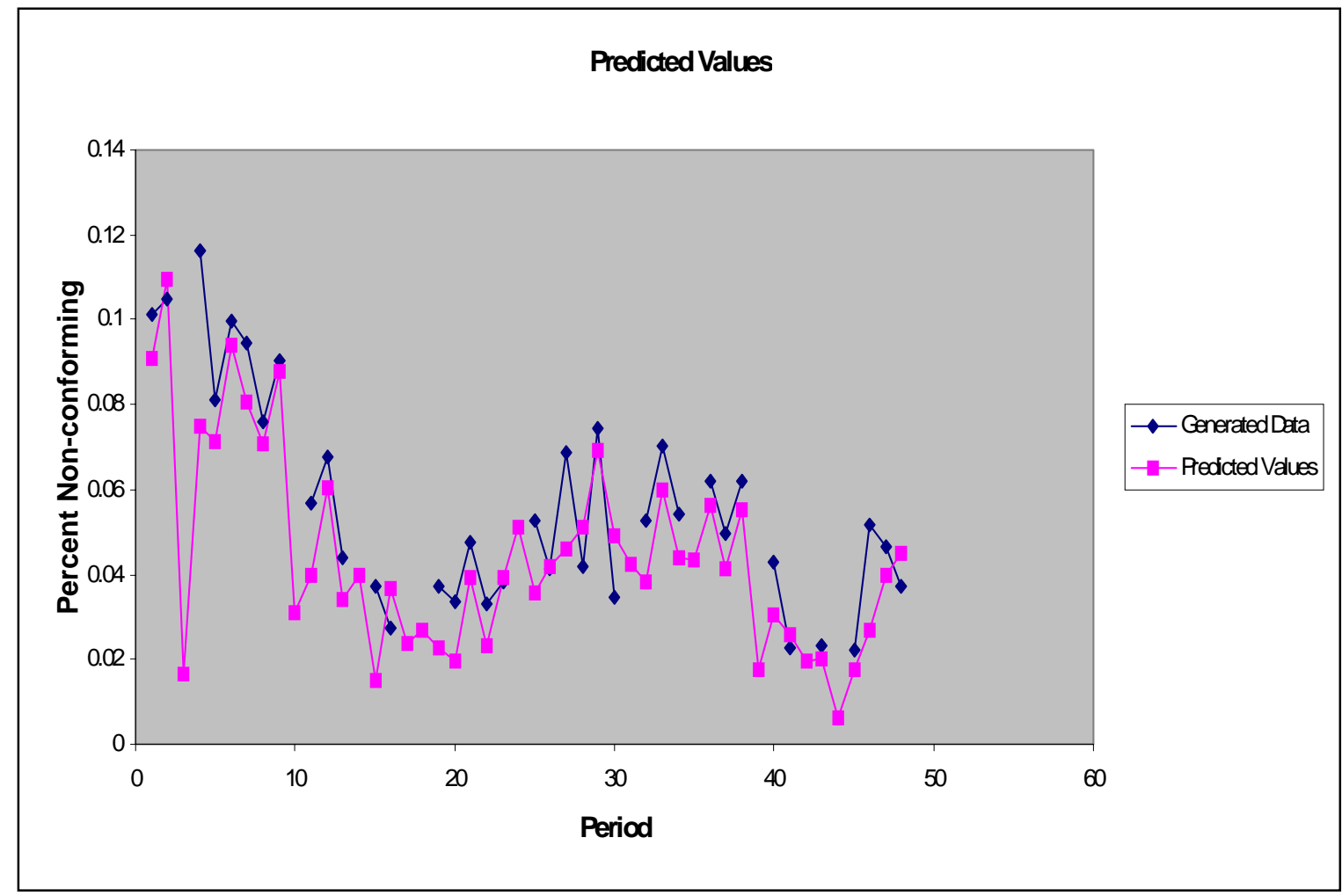

Figure 4.5.1(a) Generated vs. Predicted values, ARMA(2,2) process, 11 observations missing

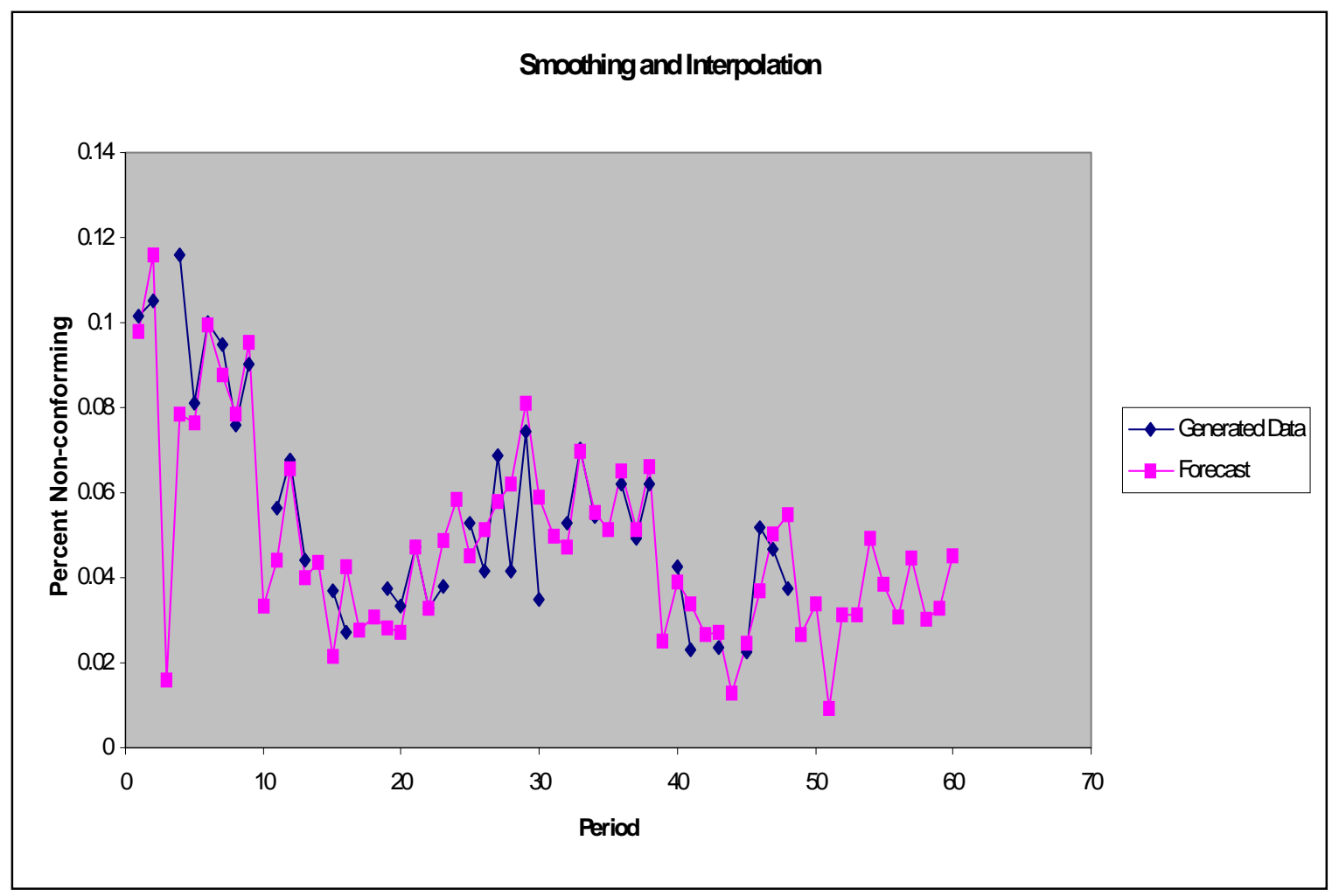

Figure 4.5.1(b) Generated vs. Forecasted values, $\operatorname{ARMA}(2,2)$ process, 11 observations missing 


\begin{tabular}{|c|c|c|c|c|c|c|c|}
\hline Period & 14 obs. Missing & Estimate & Forecast & Period & 14 obs. Missing & Estimate & Forecast \\
\hline 1 & 0.101417725 & 0.091112 & 0.100457 & 31 & & 0.020511 & 0.02934 \\
\hline 2 & 0.104934133 & 0.109469 & 0.11831 & 32 & 0.052658789 & 0.032455 & 0.043529 \\
\hline 3 & & 0.016617 & 0.018301 & 33 & 0.070499177 & 0.056105 & 0.068778 \\
\hline 4 & 0.116023022 & 0.074885 & 0.081139 & 34 & & 0.00636 & 0.015887 \\
\hline 5 & 0.080922007 & 0.071516 & 0.078815 & 35 & & -0.00156 & 0.006927 \\
\hline 6 & 0.099886633 & 0.093919 & 0.101881 & 36 & 0.0620485 & 0.046588 & 0.057263 \\
\hline 7 & 0.094682663 & 0.080712 & 0.090227 & 37 & 0.049443655 & 0.033627 & 0.04606 \\
\hline 8 & 0.07593737 & 0.070697 & 0.080794 & 38 & 0.062172423 & 0.050848 & 0.064539 \\
\hline 9 & 0.090285713 & 0.087723 & 0.098105 & 39 & & 0.011933 & 0.022499 \\
\hline 10 & & 0.030924 & 0.035945 & 40 & 0.042668614 & 0.02912 & 0.041191 \\
\hline 11 & 0.056614679 & 0.039837 & 0.046722 & 41 & 0.022828031 & 0.023205 & 0.035204 \\
\hline 12 & 0.067695322 & 0.060475 & 0.068162 & 42 & & 0.036986 & 0.048087 \\
\hline 13 & 0.044159983 & 0.033847 & 0.04268 & 43 & 0.023460506 & 0.022386 & 0.03364 \\
\hline 14 & & 0.03987 & 0.046207 & 44 & & 0.016118 & 0.026451 \\
\hline 15 & 0.037047144 & 0.014802 & 0.024294 & 45 & 0.022340746 & 0.020601 & 0.031128 \\
\hline 16 & 0.027258606 & 0.036536 & 0.044997 & 46 & 0.051637085 & 0.031474 & 0.04486 \\
\hline 17 & & 0.023539 & 0.030109 & 47 & & 0.023537 & 0.034875 \\
\hline 18 & & 0.026992 & 0.033502 & 48 & 0.037209459 & 0.039144 & 0.050266 \\
\hline 19 & 0.037437339 & 0.022684 & 0.030833 & 49 & & & 0.060345 \\
\hline 20 & 0.033519587 & 0.019885 & 0.029549 & 50 & & & 0.063674 \\
\hline 21 & 0.047304722 & 0.03911 & 0.049685 & 51 & & & 0.034694 \\
\hline 22 & 0.032965677 & 0.023242 & 0.035196 & 52 & & & 0.056139 \\
\hline 23 & 0.038172224 & 0.039422 & 0.051237 & 53 & & & 0.049995 \\
\hline 24 & & 0.050894 & 0.060983 & 54 & & & 0.070646 \\
\hline 25 & 0.052574234 & 0.035393 & 0.047391 & 55 & & & 0.055769 \\
\hline 26 & 0.041473637 & 0.041936 & 0.053868 & 56 & & & 0.053052 \\
\hline 27 & 0.068509549 & 0.046154 & 0.06057 & 57 & & & 0.061916 \\
\hline 28 & 0.041733999 & 0.051267 & 0.064624 & 58 & & & 0.044784 \\
\hline 29 & & 0.051081 & 0.061507 & 59 & & & 0.055074 \\
\hline 30 & 0.034715071 & 0.038088 & 0.048035 & 60 & & & 0.072615 \\
\hline
\end{tabular}

Table 4.5.2 Forecasting fraction non-conforming for data generated using $\operatorname{ARMA}(2,2)$ process $(14$ observations missing) 


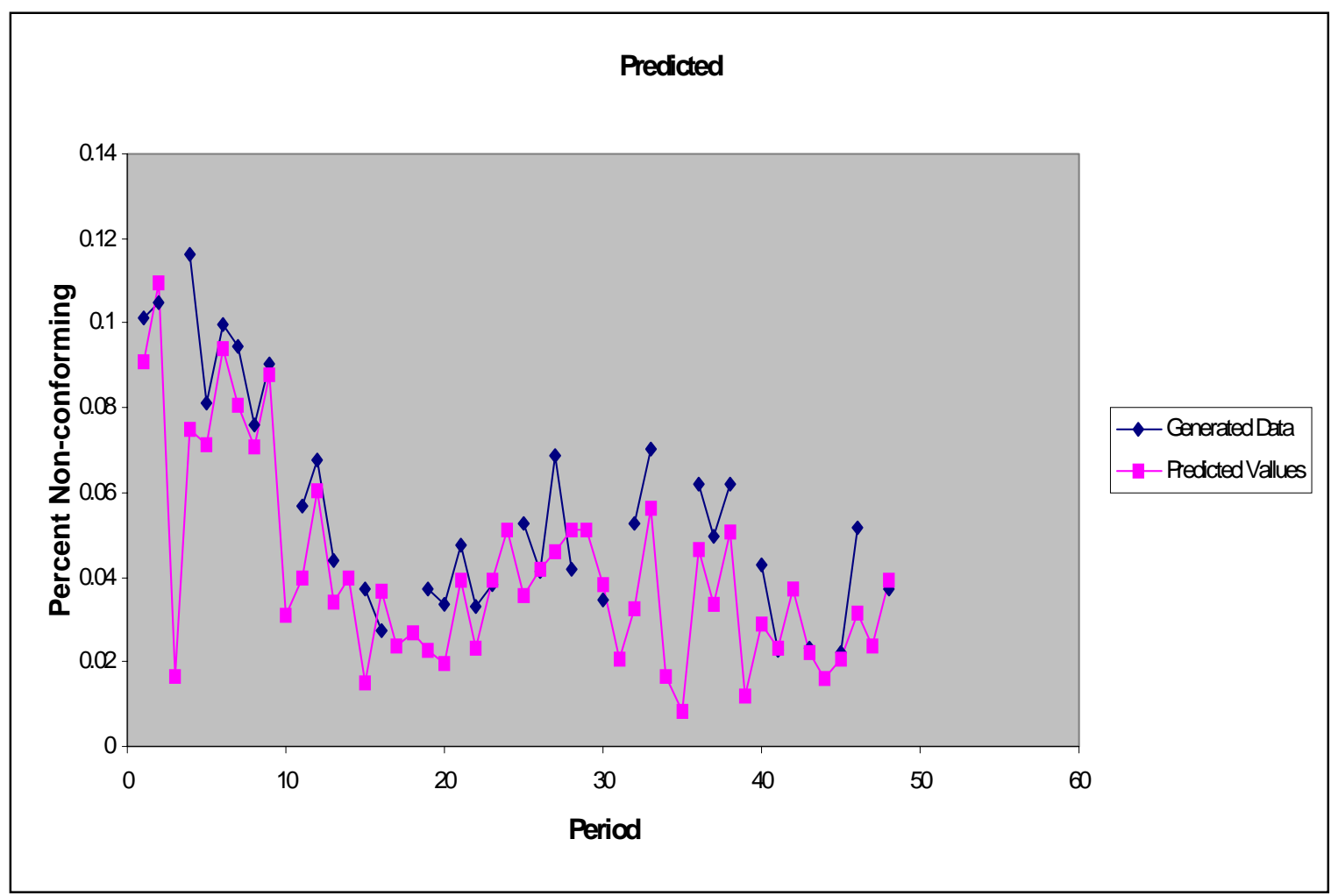

Figure 4.5.2(a) Generated vs. Predicted values, $\operatorname{ARMA}(2,2)$ process, 14 observations missing

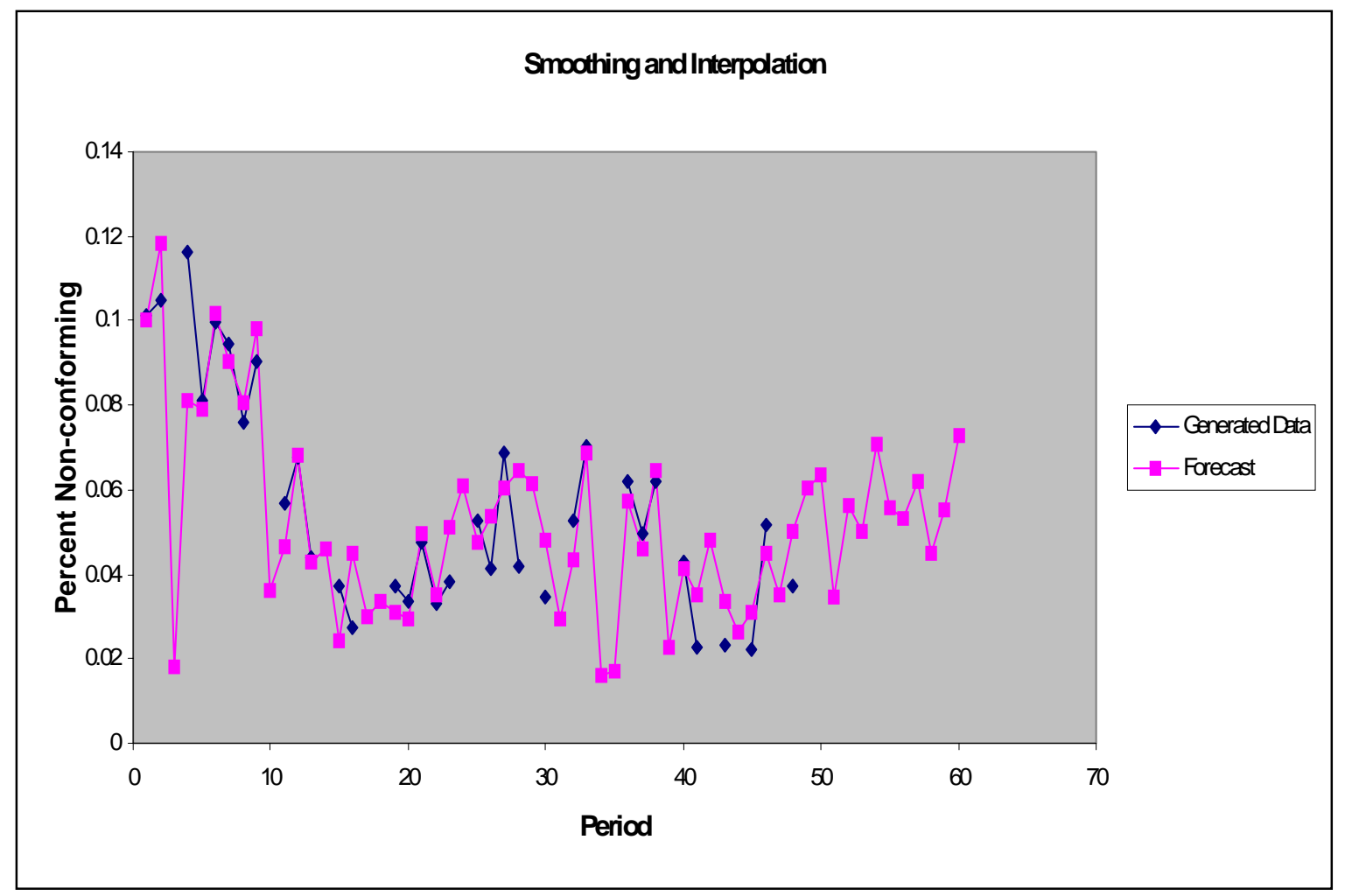

Figure 4.5.2(b) Generated vs. Forecasted values, ARMA(2,2) process, 14 observations missing 


\begin{tabular}{|c|c|c|c|c|c|c|c|}
\hline Period & 12 obs. Missing & Estimate & Forecast & Period & 12 obs. Missing & Estimate & Forecast \\
\hline 1 & 0.101417725 & 0.091112 & 0.097957 & 31 & & 0.042256 & 0.049574 \\
\hline 2 & 0.104934133 & 0.109469 & 0.11581 & 32 & 0.052658789 & 0.038402 & 0.047305 \\
\hline 3 & & 0.016617 & 0.015801 & 33 & 0.070499177 & 0.059791 & 0.069884 \\
\hline 4 & 0.116023022 & 0.074885 & 0.078639 & 34 & 0.054464728 & 0.043861 & 0.055131 \\
\hline 5 & 0.080922007 & 0.071516 & 0.076315 & 35 & & 0.04343 & 0.051514 \\
\hline 6 & 0.099886633 & 0.093919 & 0.099381 & 36 & 0.0620485 & 0.056242 & 0.065149 \\
\hline 7 & 0.094682663 & 0.080712 & 0.087727 & 37 & 0.049443655 & 0.041285 & 0.051099 \\
\hline 8 & 0.07593737 & 0.070697 & 0.078294 & 38 & 0.062172423 & 0.0554 & 0.065967 \\
\hline 9 & 0.090285713 & 0.087723 & 0.095605 & 39 & & 0.017781 & 0.024943 \\
\hline 10 & & 0.030924 & 0.033445 & 40 & 0.042668614 & 0.030299 & 0.038835 \\
\hline 11 & 0.056614679 & 0.039837 & 0.044222 & 41 & 0.022828031 & 0.025768 & 0.033978 \\
\hline 12 & 0.067695322 & 0.060475 & 0.065662 & 42 & & 0.019874 & 0.026797 \\
\hline 13 & 0.044159983 & 0.033847 & 0.04018 & 43 & 0.023460506 & 0.019995 & 0.027409 \\
\hline 14 & & 0.03987 & 0.043707 & 44 & & 0.006119 & 0.012711 \\
\hline 15 & 0.037047144 & 0.014802 & 0.021794 & 45 & 0.022340746 & 0.01731 & 0.024462 \\
\hline 16 & 0.027258606 & 0.036536 & 0.042497 & 46 & 0.051637085 & 0.027037 & 0.036922 \\
\hline 17 & & 0.023539 & 0.027609 & 47 & & 0.017872 & 0.025955 \\
\hline 18 & & 0.026992 & 0.031002 & 48 & 0.037209459 & 0.03362 & 0.042101 \\
\hline 19 & 0.037437339 & 0.022684 & 0.028333 & 49 & & & 0.031779 \\
\hline 20 & 0.033519587 & 0.019885 & 0.027049 & 50 & & & 0.037037 \\
\hline 21 & 0.047304722 & 0.03911 & 0.047185 & 51 & & & 0.01095 \\
\hline 22 & 0.032965677 & 0.023242 & 0.032696 & 52 & & & 0.031468 \\
\hline 23 & 0.038172224 & 0.039422 & 0.048737 & 53 & & & 0.030148 \\
\hline 24 & & 0.050894 & 0.058483 & 54 & & & 0.046478 \\
\hline 25 & 0.052574234 & 0.035393 & 0.044891 & 55 & & & 0.034157 \\
\hline 26 & 0.041473637 & 0.041936 & 0.051368 & 56 & & & 0.024637 \\
\hline 27 & 0.068509549 & 0.046154 & 0.05807 & 57 & & & 0.037061 \\
\hline 28 & 0.041733999 & 0.051267 & 0.062124 & 58 & & & 0.020784 \\
\hline 29 & 0.074192064 & 0.06937 & 0.08091 & 59 & & & 0.013911 \\
\hline 30 & 0.034715071 & 0.049303 & 0.058774 & 60 & & & 0.040397 \\
\hline
\end{tabular}

Table 4.5.3 Forecasting fraction non-conforming for data generated using $\operatorname{ARMA}(2,2)$ process (12 observations missing) 


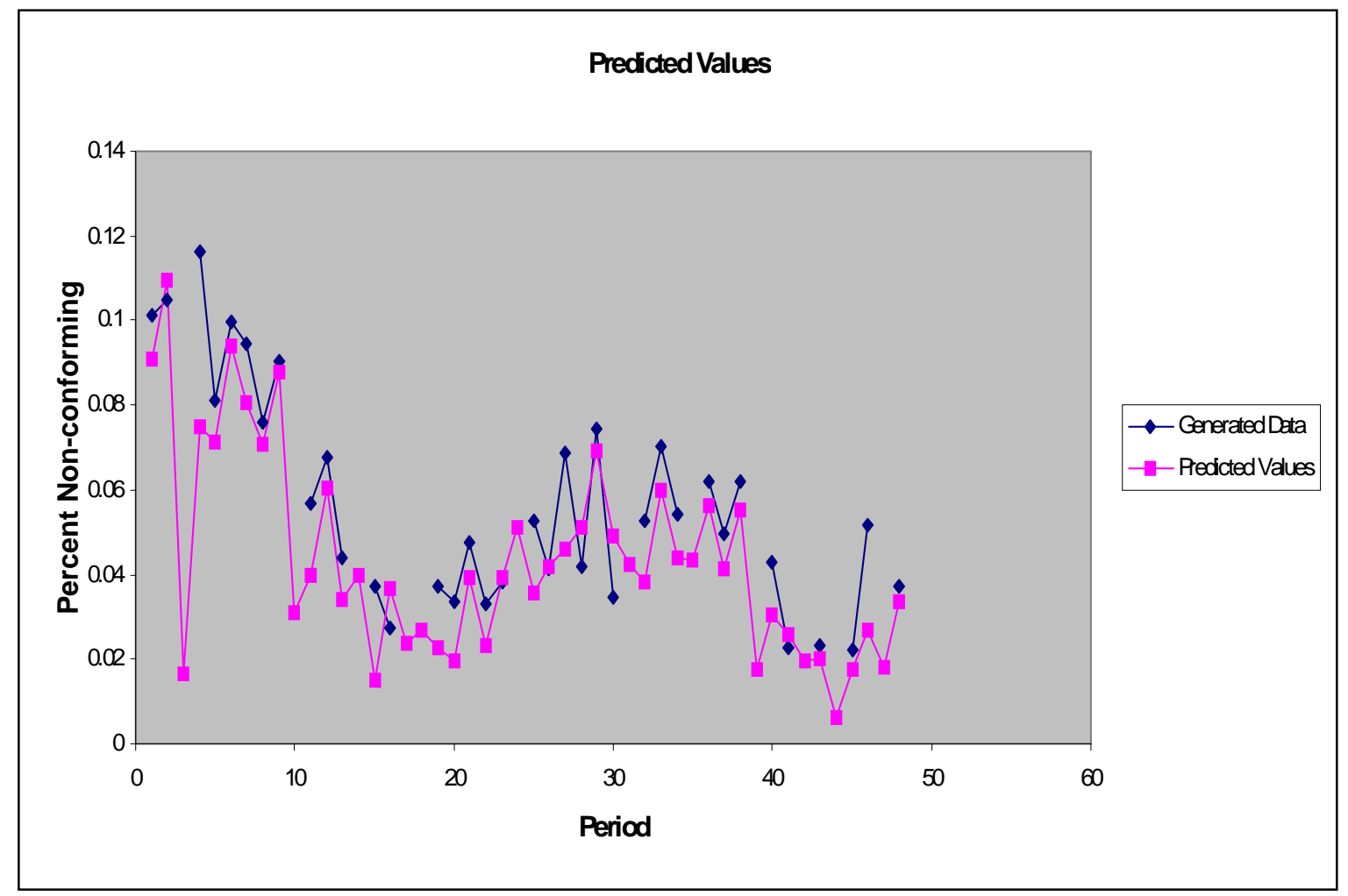

Figure 4.5.3(a) Generated vs. Predicted values, ARMA(2,2) process, 12 observations missing

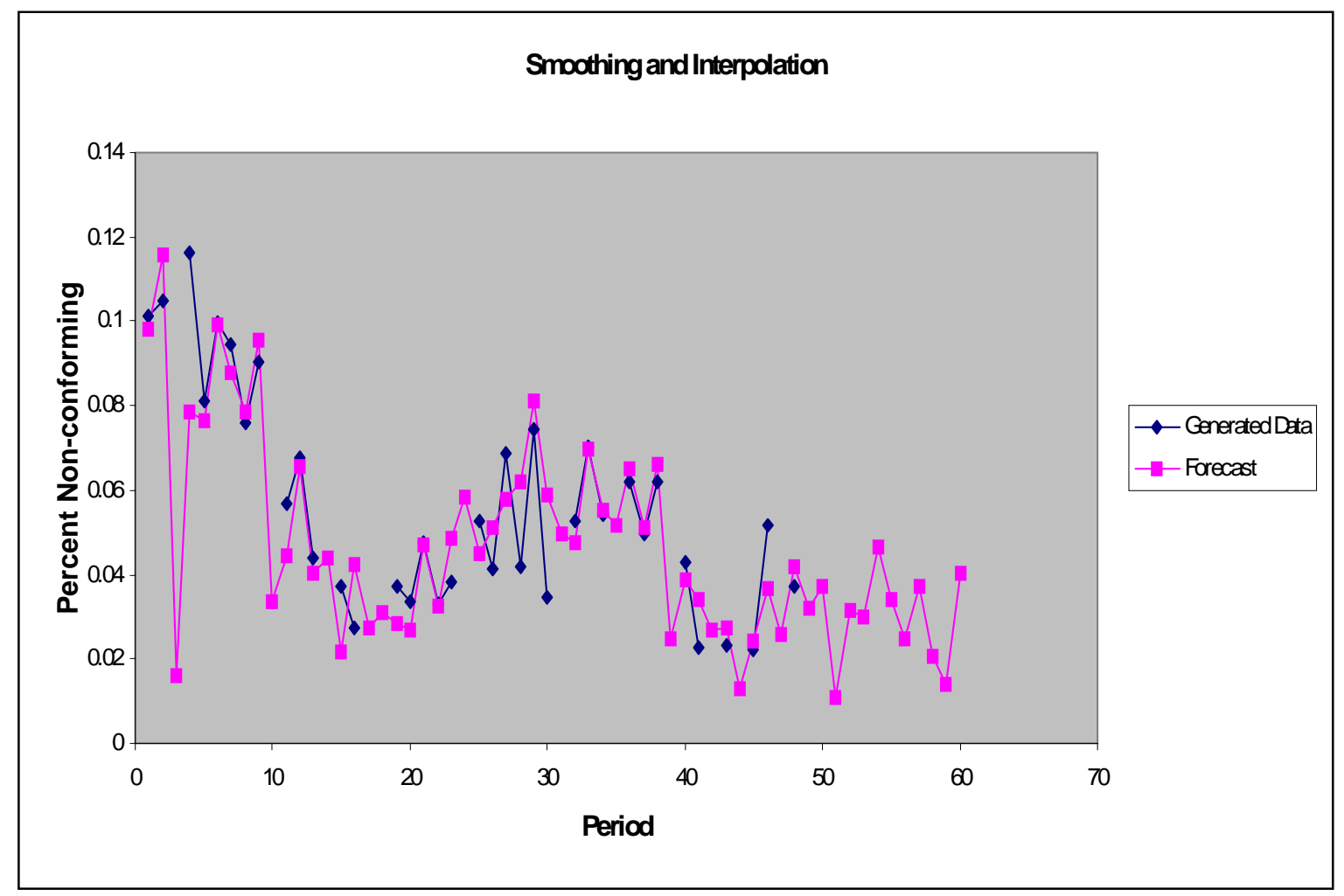

Figure 4.5.3(a) Generated vs. Forecasted values, $\operatorname{ARMA}(2,2)$ process, 12 observations missing 


\section{CONCLUSIONS AND FUTURE RESEARCH}

\subsection{CONCLUSIONS}

The results using Holt-Winters method for data generated using Autoregressive, Moving Average and Autoregressive Moving Average process illustrate that the method gives acceptable results even with some of the observations missing. This is further confirmed by performing the tracking signal analysis which shows that for almost all the methods, only a few number of forecasts are biased. The maximum number of missing observations considered is 18 which is almost $40 \%$ of the total number of observations. Thus based on the results obtained, it can be concluded that the Holt-Winters method for missing observations is good for a substantial number of observations missing.

It can be concluded from the data generated and the forecast observed that work-inprocess quality is affected by the pattern of seasonality. This seasonal pattern is fairly predictable using the Holt-Winters method, unless otherwise influenced by some extraordinary conditions. For making the forecast of percent non-conforming, the past historical data is analyzed in this research to explore a pattern of fluctuation, which has been observed to be a good guide for the future forecasts. Curves plotted after generating the forecasts indicate reduced fluctuations; the curves are smoothed and show seasonal regularity. In other words, when an average fraction non-confirming is estimated using Holt-Winters method, the effects of WIP quality fluctuations are smoothened. An important decision, which has to be taken in time, is that of adjusting the process with the fluctuations in the WIP quality. This method can be applied to scenarios where there is change in demand over season and also where the level of the demand changes over time simultaneously. Practically, this method has been applied in several instances with appropriate 
results, although the assumption of missing observations was not considered in all of these cases. The use of additive and multiplicative versions of this method has been made for forecasting for incoming calls to telemarketing centers for the purposes of planning and budgeting. Another instance of the use of this method has been for forecasting monthly claims for the Workers Compensation Board of the University of British Columbia. This was done with the aim of developing software for future use by non-expert staff.

\subsection{FUTURE RESEARCH}

There is not much research done specifically in the field of quality control with the assumption of variable percent non-conforming. This approach provides a much more realistic estimate for the percent non-conforming than assuming it to be a constant value. The research opportunities using this approach are endless. The effectiveness of the different approaches to inspection can be compared using many distributions (including beta) for percent nonconforming. Also, the effectiveness of various sampling plans can be compared.

This research uses Autoregressive, Moving Average and Autoregressive Moving Average process for generating values of fraction non-conforming for all the periods except the first one. Moreover, investigations comparing the used research methodology can be applied to obtain optimal results by interpolating missing observations in corresponding seasonal Autoregressive Integrated Moving Average (ARIMA) process. This research deals only with additive models, so similar model could be developed considering multiplicative models and the same approach could be applied to those.

Another major issue on work-in-process quality that can be targeted is corrective control of process conditions for achieving a target quality. So, a causal forecasting model that will be 
based on multiple regression models can be used to predict a WIP quality under the current process conditions. This will require to find the most significant process variables affecting quality, set of process conditions and quality indices of WIP. Further, the most stable and corrective process conditions for achieving a target quality can also be found out using this approach. A computer model to implement these systems can also be developed. Most statistical time series forecasting methods are based upon some assumptions on the data generating process. This research presents a similar approach in which assumptions are made regarding the data generation. These assumptions, however, may not be necessarily satisfied in practical situations. So robustness properties of some forecasting methods for seasonal time series could be investigated. These investigations might include whether the various methods have reasonably good forecasting performances for a wide class of time series to which the methods are likely to be

used. 


\section{References}

1. Cipra, Trujillo, and Rubio, "Holt-Winters Method with Missing Observations", Management Science, 41 (1991), 174-178.

2. Wright. David J, "Forecasting Data Published at Irregular Time Intervals", Management Science, 36 (1986), 499-510.

3. Montgomery. Douglas C, "Forecasting and Time Series Analysis", McGraw-Hill Book Company, New York, 1990.

4. Box. G. E. P., and G. M. Jenkins, "Time Series Analysis, Forecasting and Control", Revised Edition, Holden-Day, San Francisco, CA, 1976.

5. Chatfield. C, and M. Yar, "Holt-Winters Forecasting: Some Practical Issues", The Statistician, 37 (1988), 129-140.

6. Gilchrist. W, “Statistical Forecasting”, J. Wiley, New York, 1976.

7. Newbold. P, "Some Recent Developments in Time Series Analysis", International Statistical Review, 49 (1981), 53-66.

8. Brown, R. G., "Smoothing, Forecasting and Prediction of Discrete Time Series", Prentice-Hall, Englewood Cliffs, N.J., 1963.

9. Croston, J. D., "Forecasting for Intermittent Demand”, Oper. Res. Quart., 23 (1972), 289-303.

10. Dunsmuir, W., “Estimation for Stationery Time Series When Data are Irregularly Spaced or Missing”, Applied Time Series Analysis, II, Academic Press, New York, 1981, 609648.

11. Jones, R.H., "Maximum Likelihood Fitting of ARMA Models to Time Series with Missing Observations", Technometrics, 22 (1980), 389-395. 
12. Rao, A.G., "A Comment on Forecasting for Intermittent Demand", Oper. Res. Quart., 24 (1973), 639-652.

13. Chunhang Chen, "Robustness Properties of some Forecasting Methods for Seasonal Time Series: A Monte Carlo Study”, International Journal of Forecasting, Vol. 13 (2), (Jan.1997), 269-280.

14. Bianchi, L., Jarrett, J., Choudhary, R., “Improving Forecasting for Telemarketing Centers by ARIMA Modeling with Intervention”, International Journal of Forecasting, Vol. 14 (4), (Oct.1998), 497-504.

15. Lawton, R., "How Should Additive Holt-Winters Estimates be Corrected?", International Journal of Forecasting, Vol. 14 (3), (Sep.1998), 393-403.

16. Kapalka, B., Puterman, M., "Forecasting Workers Compensation Claims”, 1996, http//:www.informs.org/Conf/WA96/TALKS/WE28.4.html 


\begin{tabular}{|c|c|c|c|c|c|c|}
\hline Period & Errors & Error Sq. & Delta $(\mathrm{t})$ & $\mathrm{Y}(\mathrm{T})$ & $\mathrm{Q}(\mathrm{T})$ & $\mathrm{Q}(\mathrm{T}) / \operatorname{Delta}(\mathrm{T})$ \\
\hline 1 & 0.009505 & $61 \mathrm{E}-05$ & 7866 & 009505 & 0.001045 & 0.13292022 \\
\hline 2 & -0.01274 & 0.000162431 & 0.008354 & -0.00324 & -0.00033 & -0.03992753 \\
\hline 4 & 0.008509 & $7.2402 \mathrm{E}-05$ & 0.008369 & 0.005269 & 0.000551 & 0.06580311 \\
\hline 5 & 0.003494 & 1.22048E-05 & 0.007882 & 0.008762 & 0.000845 & 0.10721196 \\
\hline 6 & 0.000823 & 6.77208E-07 & 0.007176 & 0.009585 & 0.000843 & 0.11745072 \\
\hline 7 & -0.00029 & $8.27853 \mathrm{E}-08$ & 0.006487 & 0.009297 & 0.00073 & 0.11249428 \\
\hline 8 & -0.00262 & 6.83891E-06 & 0.0061 & 0.006682 & 0.000395 & 0.06479815 \\
\hline 9 & -0.00182 & 3.29789E-06 & 0.005671 & 0.004866 & 0.000174 & 0.03070236 \\
\hline 11 & 0.004814 & 2.31764E-05 & 0.005586 & 0.00968 & 0.000638 & 0.11424526 \\
\hline 12 & 0.003809 & $1.45095 \mathrm{E}-05$ & 0.005408 & 0.01349 & 0.000955 & 0.17663399 \\
\hline 13 & -0.00542 & 2.94116E-05 & 0.005409 & 0.008066 & 0.000317 & 0.05867137 \\
\hline 15 & 0.014582 & 0.000212621 & 0.006327 & 0.022648 & 0.001744 & 0.27562531 \\
\hline 18 & -0.01458 & 12701 & 0.007152 & 0.008064 & 0.000111 & 0.01551803 \\
\hline 19 & 0.006612 & $4.3714 \mathrm{E}-05$ & 0.007098 & 0.014675 & 0.000761 & 0.10721588 \\
\hline 20 & 0.003633 & 1.31985E-05 & 0.006752 & 0.018308 & 0.001048 & 0.15525411 \\
\hline 21 & -0.00104 & $7 \mathrm{E}-06$ & 0.00618 & 0.017272 & 0.00084 & 0.13588698 \\
\hline 22 & -0.00043 & 1.81475E-07 & 0.005605 & 0.016846 & 0.000713 & 0.1272536 \\
\hline 23 & 0.000365 & 1.33436E-07 & 0.005081 & 0.017211 & 0.000678 & 0.13352821 \\
\hline 24 & -0.00249 & 6.21254E-06 & 0.004822 & 0.014719 & 0.000361 & 0.07493651 \\
\hline 26 & -0.00351 & 1.23335E-05 & 0.004691 & 0.011207 & -2.6E-05 & -0.00553801 \\
\hline 27 & 0.012719 & 0.000161785 & 0.005494 & 0.023926 & 0.001249 & 0.22726553 \\
\hline 29 & -0.00202 & 4.07795E-06 & 0.005146 & 0.021907 & 0.000922 & 0.17910915 \\
\hline 30 & 0.00 & 3.09 & 88 & 466 & 385 & 0.26707101 \\
\hline 31 & \begin{tabular}{|l|}
0.006851 \\
\end{tabular} & 4.69374E-05 & 0.005354 & 0.034317 & 0.001932 & 0.360858 \\
\hline 32 & -0.00734 & 5.38041E-05 & 0.005552 & 0.026982 & 0.001005 & 0.18106945 \\
\hline 34 & 0.016842 & 0.000283645 & 0.006681 & 0.043824 & 0.002589 & 0.38750669 \\
\hline 35 & -0.00924 & 8.54104E-05 & 0.006937 & 0.034582 & 0.001406 & 0.20266102 \\
\hline 36 & 0.006157 & 3.79058E-05 & 0.006859 & 0.040739 & 0.001881 & 0.27423048 \\
\hline 37 & -0.00745 & $5.54608 \mathrm{E}-05$ & 0.006918 & 0.033291 & 0.000948 & 0.13705855 \\
\hline 38 & 0.000432 & 1.86717E-07 & 0.006269 & 0.033724 & 0.000897 & 0.14300627 \\
\hline 39 & -0.00579 & 3.35138E-05 & 0.006221 & 0.027934 & 0.000228 & 0.03664629 \\
\hline 41 & 5.16E-05 & 2.66463E-09 & 0.005604 & 0.027986 & 0.00021 & 0.0375336 \\
\hline 42 & 0.000967 & 9.35623E-07 & 0.005141 & 0.028953 & 0.000286 & 0.05564362 \\
\hline 46 & 0.028431 & 0.000808303 & 0.00747 & 0.057384 & 0.003101 & 0.41508094 \\
\hline 47 & 0.012234 & 0.000149682 & 0.007946 & 0.069618 & 0.004014 & 0.50513964 \\
\hline \multirow[t]{5}{*}{48} & 0.009372 & 8.78277E-05 & 0.008089 & 0.07899 & 0.00455 & 0.56247477 \\
\hline & SSE & 0.002757916 & & & & \\
\hline & SSE/34 & 8.11152E-05 & & & & \\
\hline & K3 & 0.42 & & & & \\
\hline & Delta(0) & 0.007683507 & & & & \\
\hline
\end{tabular}

Table A.1.1 Tracking Signal Analysis \{ AR(1) process, 12 observations missing \} 


\begin{tabular}{|c|c|c|c|c|c|c|}
\hline Period & rrors & Error Sq. & Delta(t) & $\mathrm{Y}(\mathrm{T})$ & $\mathrm{Q}(\mathrm{T})$ & $\mathrm{Q}(\mathrm{T}) / \operatorname{Delta}(\mathrm{T})$ \\
\hline 1 & .005 & 49547E-05 & .011086 & -0.005 & -0.0005 & -0.04506083 \\
\hline 2 & 4 & 2 & 2 & 4 & 5 & -0.27720341 \\
\hline 4 & -0.01326 & 5731 & 189 & -0.0503 & -0.00461 & 85008 \\
\hline 5 & -0.01761 & 0.000310024 & 0.013631 & -0.0679 & -0.00591 & -0.43383043 \\
\hline 6 & -0.01902 & 0.00036159 & 0.01417 & -0.08692 & -0.00722 & -0.50980995 \\
\hline 7 & 993 & 7305 & 4746 & -0.10685 & -0.00849 & 705 \\
\hline 8 & -0.01841 & 0.000338961 & 0.015112 & -0.12526 & -0.00949 & 71627 \\
\hline 9 & -0.0162 & 0.000262444 & 0.015221 & -0.14146 & -0.01016 & -0.66 \\
\hline 11 & -0.0038 & 1.44168E-05 & 0.014079 & -0.14526 & -0.00952 & -0.6 \\
\hline 12 & 806 & $E-05$ & 3477 & 332 & 338 & 978 \\
\hline 13 & -0.01546 & 0.000238989 & 0.013675 & -0.16878 & -0.00998 & -0.73007327 \\
\hline 15 & 0.008846 & 7.82468E-05 & 0.013192 & -0.15993 & -0.0081 & -0.61406729 \\
\hline 18 & 014445 & 0.000208646 & 0.013317 & -0.14549 & -0.00585 & 99954 \\
\hline 20 & 6 & 7 & 6 & 918 & -0.0 & 63 \\
\hline 21 & 0.00573 & 3.28326E-05 & 0.012828 & -0.12345 & -0.0027 & -0.21 \\
\hline 22 & 000538 & 2.89E-07 & 0.011599 & -0.12292 & -0.00237 & -0.20448681 \\
\hline 24 & 0.013329 & 0.000177663 & 0.011772 & -0.10959 & -0.0008 & -0.06810317 \\
\hline 26 & 0893 & 18E-07 & 0.010684 & -0.10869 & -0.00063 & 899 \\
\hline 27 & 3 & 38 & 39 & 46 & 54 & 95 \\
\hline 29 & 45 & 1.98365E-07 & 069 & 391 & 814 & 87 \\
\hline 31 & 0.010482 & 0.00010988 & 0.010111 & -0.08342 & 0.001781 & 0.17616027 \\
\hline 32 & 0.010995 & 0.00012089 & 0.010199 & -0.07243 & 0.002702 & 0.26497338 \\
\hline 34 & 0.0175 & 0.000306408 & 0.01093 & -0.08993 & 0.000682 & 0.06 \\
\hline 35 & 1 & 6. & 58 & 72 & 35 & 19 \\
\hline 36 & 0.00533 & 2.84124E-05 & 0.010125 & -0.07639 & 0.001824 & 0.18016636 \\
\hline 37 & -0.00573 & 3.28789E-05 & 0.009686 & -0.08213 & 0.001068 & 0.11030046 \\
\hline 38 & -0.00072 & $5.1948 \mathrm{E}-07$ & 0.008789 & -0.08285 & 0.000889 & 0.10119567 \\
\hline 39 & 77 & E-05 & 008487 & -0.08862 & 0.000224 & 812 \\
\hline 41 & 0.001784 & 3.18347E-06 & 0.007817 & -0.08683 & 0.00038 & 0.0485718 \\
\hline 42 & 0.000817 & 6.67363E-07 & 0.007117 & -0.08602 & 0.000423 & 0.0594928 \\
\hline 46 & 0.026098 & 0.00068113 & 0.009015 & -0.05992 & 0.002991 & 0.33176681 \\
\hline \multirow[t]{4}{*}{47} & 0.010285 & 0.000105787 & 0.009142 & -0.04963 & 0.00372 & $0.4 C$ \\
\hline & SSE & 0.005703281 & & & & \\
\hline & SSE/30 & 0.000190109 & & & & \\
\hline & Delta(0) & 0.011762785 & & & & \\
\hline
\end{tabular}

Table A.1.2 Tracking Signal Analysis \{ AR(1) process, 16 observations missing \} 


\begin{tabular}{|c|c|c|c|c|c|c|}
\hline Period & rors & Erro & elta(t) & $Y(T)$ & $(\mathrm{T})$ & $Q(1$ \\
\hline 2 & 3 & 1 & 1 & 3 & 1 & 2 \\
\hline 4 & 11 & 227 & 517 & -0.02125 & .00201 & 541 \\
\hline 5 & 874 & 1121 & 010439 & -0.03999 & -0.00369 & 3163 \\
\hline 7 & 035 & 037 & 43 & 033 & 35 & 295 \\
\hline 8 & 648 & 71633 & 035 & 681 & 556 & 956 \\
\hline 9 & 837 & E-05 & 615 & 998 & 432 & 022 \\
\hline 11 & 935 & 45 & 489 & -0.0 & 583 & 891 \\
\hline 12 & 08 & 859 & 948 & -0.09541 & 85 & 189 \\
\hline 13 & 586 & $E-05$ & 34 & 127 & 5 & 602 \\
\hline 15 & 018 & 2282E-06 & 0.010385 & -0.10307 & -0.00626 & 9243 \\
\hline 18 & 005844 & 3.41554E-05 & 0.009931 & -0.09722 & -0.00505 & -0.50828426 \\
\hline 19 & $=-05$ & 1.37447E-09 & 942 & -0.09719 & 454 & -0.507 \\
\hline 20 & 064 & CL나 & 0.008112 & -0.09783 & & 5292 \\
\hline 22 & 575 & 582 & 358 & 225 & 18 & 985 \\
\hline 23 & 015633 & 0.000244383 & 0.009536 & -0.06662 & -0.0004 & -0.04154514 \\
\hline 24 & 005082 & 2.58292E-05 & 0.00909 & -0.06154 & 0.000152 & 0.01668636 \\
\hline 26 & -0.00011 & 1.24324E-08 & 92 & -0.06165 & 25 & 62 \\
\hline $2 t$ & - & (. & 663 & -0.03875 & 403 & 761 \\
\hline 29 & .00193 & $70922 \mathrm{E}-06$ & 0.008889 & -0.04068 & 197 & 479 \\
\hline 30 & 0.000957 & 9.16724E-07 & 0.008096 & -0.03972 & 0.001869 & 0.23081009 \\
\hline 31 & 005379 & 2.89326E-05 & 0.007824 & -0.03434 & 0.00222 & 0.28368777 \\
\hline 34 & 002467 & 6 & 39 & -0.031 & 4 & 0.30 \\
\hline 35 & 385 & 8457 & 8 & -0. & 8 & 1 \\
\hline 37 & 0.01499 & 0.000224701 & 0.008878 & -0.0005 & 0.004792 & 0.53975036 \\
\hline 38 & -0.00619 & 3.83531E-05 & 0.008609 & -0.00669 & 0.003693 & 0.42898738 \\
\hline 39 & 0605 & 65561E-05 & 53 & -0.01274 & 19 & 001 \\
\hline 41 & 048 & $7 \mathrm{E}-05$ & 997 & -0.01753 & 968 & 457 \\
\hline 42 & -0.00467 & 2.17808E-05 & 0.007664 & -0.0222 & 0.001304 & 0.17016909 \\
\hline 46 & 0.021121 & 0.000446101 & 0.00901 & -0.00108 & 0.003286 & 0.36470044 \\
\hline \multirow[t]{4}{*}{47} & 0.012849 & 0.000165095 & 0.009394 & 0.011769 & 0.004242 & 0.45159789 \\
\hline & SSE & 8957 & & & & \\
\hline & SSE/28 & 0.000117911 & & & & \\
\hline & Delta(0) & 0.009263704 & & & & \\
\hline
\end{tabular}

Table A.1.3 Tracking Signal Analysis \{ AR(1) process, 18 observations missing \} 


\begin{tabular}{|c|c|c|c|c|c|c|}
\hline Period & Error & error Sq. & delta(T) & $\mathrm{Y}(\mathrm{T})$ & $Q(T)$ & $\mathrm{Q}(\mathrm{T}) / \operatorname{Delta}(\mathrm{T})$ \\
\hline 1 & -0.00124 & 1.53637E-06 & 0.007104 & -0.00124 & -0.00012 & -0.01744907 \\
\hline 2 & -0.0036 & 1.29611E-05 & 0.006753 & -0.00484 & -0.00047 & -0.06982916 \\
\hline 3 & -0.00315 & 9.91478E-06 & 0.006393 & -0.00799 & -0.00074 & -0.11564499 \\
\hline 5 & 0.019209 & 0.000368993 & 0.007674 & 0.011221 & 0.001256 & 0.16360308 \\
\hline 6 & 0.010017 & 0.000100332 & 0.007909 & 0.021237 & 0.002132 & 0.26953584 \\
\hline 7 & 0.002049 & 4.19772E-06 & 0.007323 & 0.023286 & 0.002123 & 0.28997383 \\
\hline 8 & -0.00013 & $1.7398 \mathrm{E}-08$ & 0.006604 & 0.023154 & 0.001898 & 0.28739719 \\
\hline 9 & -0.00252 & 6.35581E-06 & 0.006195 & 0.020633 & 0.001456 & 00889 \\
\hline 10 & -0.00118 & 1.39121E-06 & 0.005694 & 0.019454 & 0.001192 & 2486 \\
\hline 11 & -0.00451 & 2.03288E-05 & 0.005575 & 0.014945 & 0.000622 & 176 \\
\hline 13 & 0.017035 & 0.000290201 & 0.006721 & 0.03198 & 0.002264 & 0.33 \\
\hline 15 & -0.02833 & 0.000802786 & 0.008882 & 0.003647 & -0.0008 & 62758 \\
\hline 16 & 0.009468 & 8.96379E-05 & 0.008941 & 0.013114 & 0.00023 & 445 \\
\hline 18 & 0.01 & 3129 & 472 & 367 & 0.00 & 34 \\
\hline 19 & 0.007595 & 5.76907E-05 & 0.009284 & 0.034962 & 0.002229 & 0.24005422 \\
\hline 20 & -0.00139 & 1.94066E-06 & 0.008495 & 0.033569 & 0.001867 & 963 \\
\hline 21 & -1. & $44 \mathrm{E}-10$ & & 555 & 0.0 & \\
\hline 23 & 0.015543 & 0.000241591 & 0.008437 & 0.049098 & 0.003065 & 0.36328559 \\
\hline 24 & -0.0002 & 3.82438E-08 & 0.007613 & 0.048903 & 0.002739 & 3348 \\
\hline 25 & -0 . & 2.30541E-05 & & 01 & 0.0 & 773 \\
\hline 26 & 0.000307 & $9.4283 \mathrm{E}-08$ & 0.006629 & 0.044408 & 0.001817 & 0.27410865 \\
\hline 29 & 0.004064 & 1.65192E-05 & 0.006373 & 0.048472 & 0.002042 & 0.32040499 \\
\hline 30 & -0.004 & 1.60363E-05 & 0.006136 & 0.044468 & 0.001437 & 2916 \\
\hline 31 & 0.000436 & $1.90278 \mathrm{E}-07$ & 0.005566 & 0.044904 & 0.001337 & 0.24023067 \\
\hline 32 & 0.00363 & 1.31789E-05 & 0.005372 & 0.048534 & 0.001566 & 0.29157122 \\
\hline 33 & -0.004 & 1.60108E-05 & 0.005235 & 0.044533 & 0.00101 & 0.19285453 \\
\hline 35 & 0.013725 & 0.00018837 & 0.006084 & 0.058258 & 0.002281 & 0.37 \\
\hline 36 & 0.004376 & 1.91464E-05 & 0.005913 & 0.062634 & 0.002491 & 0.421185 \\
\hline 37 & 0.000235 & 5.51736E-08 & 0.005345 & 0.062868 & 0.002265 & 0.42372842 \\
\hline 39 & -0.00375 & 1.40269E-05 & 0.005185 & 0.059123 & 0.001664 & 0.32089797 \\
\hline 40 & -0.00133 & $1.76338 \mathrm{E}-06$ & 0.0048 & 0.057795 & 0.001365 & 0.28435295 \\
\hline 42 & 0.005745 & 3.30021E-05 & 0.004894 & 0.06354 & 0.001803 & 0.36835452 \\
\hline 44 & 0.010082 & 0.000101642 & 0.005413 & 0.073622 & 0.002631 & 0.48600006 \\
\hline 45 & 0.006278 & 3.94084E-05 & 0.005499 & 0.079899 & 0.002995 & 0.54467326 \\
\hline 46 & -0.00617 & 3.80409E-05 & 0.005566 & 0.073732 & 0.002079 & 0.37351476 \\
\hline 47 & -0.009 & 8.10599E-05 & 0.00591 & 0.064728 & 0.000971 & 0.16427145 \\
\hline \multirow[t]{4}{*}{48} & -0.00881 & 7.75491E-05 & 0.0062 & 0.055922 & $-6.9 E-06$ & -0.00110731 \\
\hline & SSE & 0.00289219 & & & & \\
\hline & SSE/35 & 8.2634E-05 & & & & \\
\hline & Delta(0) & 0.007755107 & & & & \\
\hline
\end{tabular}

Table A.2.1 Tracking Signal Analysis \{ AR(2) process, 11 observations missing \} 


\begin{tabular}{|c|c|c|c|c|c|c|}
\hline Period & Error & error Sq. & $\operatorname{delta}(\mathrm{T})$ & $\mathrm{Y}(\mathrm{T})$ & $\mathrm{Q}(\mathrm{T})$ & $\mathrm{Q}(\mathrm{T}) / \operatorname{Delta}(\mathrm{T})$ \\
\hline 1 & -0.00624 & 3.89314E-05 & 0.010045 & -0.00624 & -0.00062 & -0.06211701 \\
\hline 2 & -0.0086 & 7.39627E-05 & 0.0099 & -0.01484 & -0.00142 & -0.14358871 \\
\hline 3 & -0.00815 & 6.64025E-05 & 0.009725 & -0.02299 & -0.00209 & -0.21534808 \\
\hline 4 & -0.00785 & 6.16523E-05 & 0.009538 & -0.03084 & -0.00267 & -0.27994363 \\
\hline 5 & -0.00815 & 6.64968E-05 & 0.009399 & -0.03899 & -0.00322 & -0.34241232 \\
\hline 6 & -0.00724 & 5.24812E-05 & 0.009184 & -0.04624 & -0.00362 & -0.3942833 \\
\hline 7 & -0.00846 & 7.15717E-05 & 0.009112 & -0.0547 & -0.0041 & -0.45052341 \\
\hline 8 & -0.00617 & 3.8058E-05 & 0.008817 & -0.06087 & -0.00431 & -0.48896797 \\
\hline 9 & -0.01063 & 0.000113078 & 0.008999 & -0.0715 & -0.00494 & -0.54935507 \\
\hline 10 & -0.00741 & 5.49535E-05 & 0.00884 & -0.07892 & -0.00519 & -0.58714366 \\
\hline 11 & -0.00957 & 9.1498E-05 & 0.008913 & -0.08848 & -0.00563 & -0.63145201 \\
\hline 13 & 0.018086 & 0.0003271 & 0.00983 & -0.07039 & -0.00326 & -0.33129248 \\
\hline 15 & 0.02965 & 0.000879119 & 0.011812 & -0.04074 & $3.4 \mathrm{E}-05$ & 0.00287754 \\
\hline 16 & 0.015017 & 0.0002255 & 0.012133 & -0.02573 & 0.001532 & 0.12629227 \\
\hline 18 & 0.021739 & 0.000472563 & 0.013093 & -0.00399 & 0.003553 & 0.27135303 \\
\hline 19 & 0.01079 & 0.000116424 & 0.012863 & 0.0068 & 0.004277 & 0.33247538 \\
\hline 20 & -0.00035 & 1.25283E-07 & 0.011612 & 0.006446 & 0.003814 & 0.32841378 \\
\hline 21 & $6.13 \mathrm{E}-05$ & 3.76345E-09 & 0.010457 & 0.006508 & 0.003438 & 0.32880777 \\
\hline 23 & 0.015751 & 0.000248085 & 0.010986 & 0.022258 & 0.00467 & 0.42503444 \\
\hline 24 & -7.3E-05 & 5.33468E-09 & 0.009895 & 0.022185 & 0.004195 & 0.42398256 \\
\hline 25 & -0.0096 & 9.21956E-05 & 0.009866 & 0.012584 & 0.002816 & 0.28539218 \\
\hline 26 & 0.000622 & 3.87167E-07 & 0.008941 & 0.013206 & 0.002596 & 0.29036514 \\
\hline 28 & -0.0061 & 3.71894E-05 & 0.008657 & 0.007107 & 0.001727 & 0.19946735 \\
\hline 29 & 0.001002 & 1.00451E-06 & 0.007892 & 0.00811 & 0.001654 & 0.20963436 \\
\hline 30 & -0.01036 & 0.000107375 & 0.008139 & -0.00225 & 0.000453 & 0.05562195 \\
\hline 32 & 0.013508 & 0.000182453 & 0.008676 & 0.011255 & 0.001758 & 0.20265891 \\
\hline 33 & 0.002059 & 4.23765E-06 & 0.008014 & 0.013314 & 0.001788 & 0.22314068 \\
\hline 35 & 0.016276 & 0.000264894 & 0.00884 & 0.029589 & 0.003237 & 0.36617067 \\
\hline 37 & -0.02201 & 0.000484334 & 0.010157 & 0.007582 & 0.000712 & 0.07014943 \\
\hline 39 & 0.002957 & 8.74637E-06 & 0.009437 & 0.010539 & 0.000937 & 0.09929025 \\
\hline 42 & 0.021944 & 0.000481558 & 0.010688 & 0.032483 & 0.003038 & 0.28422985 \\
\hline 44 & 0.01411 & 0.000199085 & 0.01103 & 0.046593 & 0.004145 & 0.37579406 \\
\hline 45 & 0.008401 & 7.05797E-05 & 0.010767 & 0.054994 & 0.004571 & 0.42449933 \\
\hline 46 & -0.00589 & 3.46366E-05 & 0.010279 & 0.049109 & 0.003525 & 0.34293715 \\
\hline \multirow[t]{4}{*}{48} & 0.001177 & 1.38624E-06 & 0.009369 & 0.050286 & 0.00329 & 0.35119467 \\
\hline & SSE & 0.004968076 & & & & \\
\hline & SSE/33 & 0.000150548 & & & & \\
\hline & $\operatorname{DELTA}(0)$ & 0.010467563 & & & & \\
\hline
\end{tabular}

Table A.2.2 Tracking Signal Analysis $\{$ AR(2) process, 13 observations missing $\}$ 


\begin{tabular}{|c|c|c|c|c|c|c|}
\hline Period & Error & error Sq. & $\operatorname{delta}(\mathrm{T})$ & $\mathrm{Y}(\mathrm{T})$ & $\mathrm{Q}(\mathrm{T})$ & $\mathrm{Q}(\mathrm{T}) / \operatorname{Delta}(\mathrm{T})$ \\
\hline 1 & .00624 & 89314E-05 & .007096 & -0.00624 & -0.00062 & 0.08792541 \\
\hline 2 & & 5 & 7 & 4 & 2 & -0.19616698 \\
\hline 3 & .00815 & $64025 \mathrm{E}-05$ & 337 & -0.02299 & 209 & 169 \\
\hline 5 & 014209 & .000201901 & .008024 & -0.00878 & -0.00046 & 31828 \\
\hline 6 & 005017 & 2.51659E-05 & 0.007723 & -0.00376 & 8.41E-05 & 0.01088989 \\
\hline 7 & 295 & $\mathrm{E}-06$ & 246 & 671 & -0. & 081 \\
\hline 8 & -0.00013 & $1.7398 \mathrm{E}-08$ & 0.006535 & -0.00685 & -0.00021 & 3815 \\
\hline 9 & -0.00752 & 5.65665E-05 & 633 & -0.01437 & -0.00094 & 504 \\
\hline 10 & -0.00618 & 3.81862E-05 & 588 & -0.02055 & -0.0 & 813 \\
\hline 11 & 51 & E-05 & 88 & 6 & -0.0 & -0 . \\
\hline 13 & 012035 & 0.000144848 & 0.007396 & -0.01802 & -0.00084 & 8706 \\
\hline 15 & 0.023333 & 0.000544451 & 0.008989 & 0.005314 & 0.001578 & 0.17553757 \\
\hline 16 & 004468 & 06E-05 & 0.008537 & 0.009781 & 0.001867 & 365 \\
\hline 10 & 2 & 5 & 09 & 4 & 5 & \\
\hline 19 & 595 & $E-06$ & 007 & 0.021629 & 604 & 959 \\
\hline 20 & -0.00139 & 1.94066E-06 & 0.007346 & 0.020236 & 0.002205 & 2762 \\
\hline 21 & $-1.4 \mathrm{E}-05$ & $2.03444 \mathrm{E}-10$ & 0.006613 & 0.020222 & 0.001983 & 0.29984719 \\
\hline 23 & 10543 & 1159 & 006 & 0.030765 & 839 & 428 \\
\hline 24 & & 8 & 5 & 9 & 35 & 42 \\
\hline 26 & 0 & $E-05$ & 439 & 55 & 35 & 27 \\
\hline 29 & 001642 & 2.6951E-06 & 0.005959 & 0.024746 & 0.001546 & 3999 \\
\hline 30 & 0.0023 & 5.27848E-06 & 0.005593 & 0.022449 & 0.001162 & 408 \\
\hline 32 & 0.010052 & .000101052 & .006039 & 0.032501 & 0.002051 & 162 \\
\hline 33 & 7 & 6 & 2 & 88 & 88 & 84 \\
\hline 35 & 0.010554 & 0.000111378 & 0.006142 & 0.040882 & 0.002521 & 0.41040272 \\
\hline 37 & 0.009096 & 8.27444E-05 & 0.006438 & 0.049978 & 0.003178 & 0.49371021 \\
\hline 39 & -0.00485 & $.3537 E-05$ & 0.006279 & 0.045127 & 0.002375 & 0.37830208 \\
\hline 42 & 97 & E-05 & 31 & 924 & 18 & 827 \\
\hline 44 & 0.005361 & 2.87455E-05 & 0.006324 & 0.058285 & 0.003162 & 0.49999504 \\
\hline 45 & 0.00 & E-06 & 0.005861 & 0.059976 & 0.003015 & 187 \\
\hline 46 & -0.01105 & 0.000122182 & 0.00638 & 0.048923 & 0.001608 & 0.25204339 \\
\hline \multirow[t]{4}{*}{48} & -0.00389 & 1.51104E-05 & 0.006131 & 035 & 0.00 & 749 \\
\hline & SSE & 0.002131823 & & & & \\
\hline & SSE/30 & 7.10608E-05 & & & & \\
\hline & Delta(0) & 0.007191565 & & & & \\
\hline
\end{tabular}

Table A.2.3 Tracking Signal Analysis $\{\mathrm{AR}(2)$ process, 16 observations missing $\}$ 


\begin{tabular}{|c|c|c|c|c|c|c|}
\hline Period & Error & error Sq. & $\operatorname{delta}(T)$ & $Y(T)$ & $Q(T)$ & $\mathrm{Q}(\mathrm{T}) / \operatorname{Delta}(\mathrm{T})$ \\
\hline 1 & .00624 & $E-05$ & 3 & -0.00624 & -0.00062 & 35239 \\
\hline 2 & .0086 & $39627 \mathrm{E}-05$ & .010321 & -0.01484 & -0.00142 & -0.13773055 \\
\hline 3 & -0.00815 & 6.64025E-05 & 0.010104 & -0.02299 & -0.00209 & -0.20727082 \\
\hline 5 & 209 & 368993 & 015 & 378 & 5 & 351 \\
\hline 6 & 010017 & 00332 & 0.01 & 0.006237 & 0.001034 & 332 \\
\hline 7 & 002049 & $\mathrm{E}-06$ & 0.010028 & 0.008286 & 0.00 & 833 \\
\hline 9 & 941 & 0.000481415 & 0.01122 & 0.030227 & 0.003216 & 5564 \\
\hline 10 & 259 & $=-06$ & 357 & 633 & 35 & 31 \\
\hline 11 & 319 & E-05 & 641 & 0.024439 & 0.002052 & 125 \\
\hline 13 & .020387 & 0.00041562 & 0.010715 & 0.044825 & 0.003886 & 0.36262031 \\
\hline 15 & 0.028395 & 0.00080625 & 0.012483 & 0.07322 & 0.006336 & 0.50759923 \\
\hline 16 & -0.00129 & $1.6651 \mathrm{E}-06$ & 0.011364 & 0.071929 & 0.005574 & 35 \\
\hline 18 & 209 & $E-06$ & 437 & 0.069838 & 807 & 346 \\
\hline 19 & 006178 & 78E-05 & 0.010011 & 0.076016 & 0.004944 & 0071 \\
\hline 20 & -0.02853 & 0.000814002 & 0.011863 & 0.047485 & 0.001597 & 0.1346099 \\
\hline 21 & 111279 & 7217 & 0.0 & 764 & 565 & 716 \\
\hline 23 & 6 & 97 & $=0$ & .0 & & 19 \\
\hline 24 & 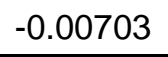 & 5 & 6 & 0.0 & 37 & 96 \\
\hline 26 & -0.00281 & 7.87921E-06 & 0.011059 & 0.067948 & 0.002497 & 0.22581613 \\
\hline 29 & -0.00474 & 2.24543E-05 & 0.010427 & 0.063209 & 0.001774 & 0.1701067 \\
\hline 30 & -0.00372 & 1.38733E-05 & 0.009756 & 0.059485 & 0.001224 & 0.125 \\
\hline 32 & 1 & 5 & 5 & 7 & 5 & 51 \\
\hline 33 & 0.0067 & 4.48966E-05 & 0.009339 & 0.05767 & 0.000895 & 0.09583412 \\
\hline 35 & 0.016952 & 0.000287373 & 0.0101 & 0.074622 & 0.002501 & 0.24758497 \\
\hline 37 & 0.014171 & 0.00020081 & 0.010507 & 0.088793 & 0.003668 & 0.34905837 \\
\hline 39 & 5 & 2.48064E-07 & 0.009507 & 0.088 & 0.00 & 0.34 \\
\hline 42 & 0.012004 & 0.000144086 & 0.009756 & 0.100299 & 0.004126 & 0.42294871 \\
\hline 44 & -0.00745 & 5.54497E-05 & 0.009525 & 0.092852 & 0.002969 & 0.31170835 \\
\hline 46 & -0.00084 & 7.13719E-07 & 0.008657 & 0.092007 & 0.002588 & 0.29890797 \\
\hline \multirow[t]{4}{*}{48} & 0.004974 & $2.47408 \mathrm{E}-05$ & 0.008289 & 0.09 & 0.002826 & 0.3 \\
\hline & SSE & 0.004644443 & & & & \\
\hline & SSE/28 & 0.000165873 & & & & \\
\hline & Delta(0) & 0.010987432 & & & & \\
\hline
\end{tabular}

Table A.2.4 Tracking Signal Analysis $\{$ AR(2) process, 18 observations missing \} 


\begin{tabular}{|c|c|c|c|c|c|c|}
\hline Period & Error & Error Sq. & $\operatorname{Delta}(\mathrm{T})$ & $\mathrm{Y}(\mathrm{T})$ & $\mathrm{Q}(\mathrm{T})$ & $\mathrm{Q}(\mathrm{T}) / \operatorname{Delta}(\mathrm{T})$ \\
\hline 1 & -0.00127 & $1.60321 \mathrm{E}-06$ & 0.007375 & -0.00127 & -0.00013 & -0.01716851 \\
\hline 2 & -0.00477 & 2.27779E-05 & 0.007115 & -0.00604 & -0.00059 & -0.083097364 \\
\hline 3 & -0.00347 & 1.20694E-05 & 0.006751 & -0.00951 & -0.00088 & -0.130283782 \\
\hline 4 & -0.00462 & $2.13858 \mathrm{E}-05$ & 0.006538 & -0.01414 & -0.00125 & -0.191800149 \\
\hline 5 & -0.00134 & 1.79484E-06 & 0.006018 & -0.01548 & -0.00126 & -0.209791433 \\
\hline 7 & 0.016834 & 0.000283384 & 0.0071 & 0.001357 & 0.000547 & 0.077056031 \\
\hline 8 & -0.00044 & 1.93798E-07 & 0.006434 & 0.000917 & 0.000448 & 0.069686473 \\
\hline 9 & -0.00186 & 3.47711E-06 & 0.005977 & -0.00095 & 0.000217 & 0.036314127 \\
\hline 10 & -0.00059 & 3.44716E-07 & 0.005438 & -0.00154 & 0.000137 & 0.025125258 \\
\hline 12 & 0.016502 & 0.000272325 & 0.006544 & 0.014967 & 0.001773 & 0.270948755 \\
\hline 13 & 0.009689 & 9.38736E-05 & 0.006859 & 0.024656 & 0.002565 & 0.373934986 \\
\hline 14 & -0.00075 & 5.58832E-07 & 0.006248 & 0.023908 & 0.002234 & 0.357495581 \\
\hline 15 & 0.000241 & 5.82389E-08 & 0.005647 & 0.02415 & 0.002034 & 0.360241321 \\
\hline 17 & 0.01151 & 0.000132482 & 0.006233 & 0.03566 & 0.002982 & 0.478374418 \\
\hline 18 & -0.00409 & 1.67504E-05 & 0.006019 & 0.031567 & 0.002274 & 0.377854976 \\
\hline 21 & 0.026904 & 0.000723836 & 0.008108 & 0.058471 & 0.004737 & 0.584302123 \\
\hline 22 & 0.016009 & 0.000256288 & 0.008898 & 0.07448 & 0.005865 & 0.659093897 \\
\hline 23 & -0.00277 & 7.68598E-06 & 0.008285 & 0.071708 & 0.005001 & 0.603579064 \\
\hline 24 & -0.00014 & 2.08141E-08 & 0.007471 & 0.071564 & 0.004486 & 0.600482532 \\
\hline 25 & -0.0114 & 0.000129946 & 0.007864 & 0.060164 & 0.002898 & 0.368484295 \\
\hline 26 & 0.000443 & 1.95852E-07 & 0.007122 & 0.060607 & 0.002652 & 0.3724085 \\
\hline 27 & -0.00251 & 6.28769E-06 & 0.00666 & 0.058099 & 0.002136 & 0.320740248 \\
\hline 28 & -0.00914 & 8.35894E-05 & 0.006909 & 0.048957 & 0.001008 & 0.145958281 \\
\hline 29 & -0.01012 & 0.000102314 & 0.007229 & 0.038842 & -0.0001 & -0.014380614 \\
\hline 31 & 0.006295 & 3.96222E-05 & 0.007136 & 0.045136 & 0.000536 & 0.075098885 \\
\hline 33 & 0.023861 & 0.000569351 & 0.008808 & 0.068997 & 0.002868 & 0.325645709 \\
\hline 34 & -0.00455 & 2.06618E-05 & 0.008382 & 0.064452 & 0.002127 & 0.253757263 \\
\hline 35 & 0.002838 & 8.05558E-06 & 0.007828 & 0.06729 & 0.002198 & 0.280815136 \\
\hline 36 & -0.00467 & 2.18528E-05 & 0.007512 & 0.062615 & 0.001511 & 0.20111464 \\
\hline 37 & -0.00654 & 4.27219E-05 & 0.007415 & 0.056079 & 0.000706 & 0.095235386 \\
\hline 38 & -0.0045 & 2.02781E-05 & 0.007124 & 0.051576 & 0.000185 & 0.026001196 \\
\hline 40 & 0.006912 & 4.77748E-05 & 0.007102 & 0.058488 & 0.000858 & 0.120788306 \\
\hline 42 & 0.002556 & 6.53158E-06 & 0.006648 & 0.061044 & 0.001028 & 0.154589038 \\
\hline 43 & -0.00188 & 3.55061E-06 & 0.006171 & 0.059159 & 0.000736 & 0.119336283 \\
\hline 44 & 0.001283 & 1.64591E-06 & 0.005683 & 0.060442 & 0.000791 & 0.139218604 \\
\hline 45 & -0.00174 & 3.02148E-06 & 0.005288 & 0.058704 & 0.000538 & 0.101771944 \\
\hline 46 & -0.00437 & 1.91017E-05 & 0.005196 & 0.054333 & 4.73E-05 & 0.009104699 \\
\hline \multirow[t]{4}{*}{48} & 0.015197 & 0.000230949 & 0.006196 & 0.06953 & 0.001562 & 0.252125095 \\
\hline & SSE & 0.003208362 & & & & \\
\hline & SSE/36 & 8.91212E-05 & & & & \\
\hline & $\operatorname{DELTA}(0)$ & 0.008053763 & & & & \\
\hline
\end{tabular}

Table A.3.1 Tracking Signal Analysis\{ARMA(1,1) process, 10 observations missing \} 


\begin{tabular}{|c|c|c|c|c|c|c|}
\hline Period & Error & Error Sq. & $\operatorname{Delta}(\mathrm{T})$ & $\mathrm{Y}(\mathrm{T})$ & $\mathrm{Q}(\mathrm{T})$ & $\mathrm{Q}(\mathrm{T}) / \operatorname{Delta}(\mathrm{T})$ \\
\hline 1 & -0.007266178 & 27973E-05 & 0.006976 & -0.00727 & -0.00073 & -0.104166353 \\
\hline 2 & -0.005772623 & 3.33232E-05 & 0.006855 & -0.01304 & -0.00123 & -0.179602005 \\
\hline 3 & -0.004474102 & 2.00176E-05 & 0.006617 & -0.01751 & -0.00156 & -0.23507223 \\
\hline 4 & -0.005624483 & 3.16348E-05 & 0.006518 & -0.02314 & -0.00196 & -0.301080274 \\
\hline 5 & -0.002339717 & $5.47428 \mathrm{E}-06$ & 0.0061 & -0.02548 & -0.002 & -0.327887779 \\
\hline 7 & 0.01583402 & 0.000250716 & 0.007073 & -0.00964 & -0.00022 & -0.030638492 \\
\hline 8 & 0.008559775 & 7.32697E-05 & 0.007222 & -0.00108 & 0.000661 & 0.091514993 \\
\hline 9 & 0.002135298 & 4.5595E-06 & 0.006713 & 0.001052 & 0.000808 & 0.120410683 \\
\hline 10 & -0.001587126 & 2.51897E-06 & 0.006201 & -0.00054 & 0.000569 & 0.09173313 \\
\hline 12 & 0.015502284 & 0.000240321 & 0.007131 & 0.014967 & 0.002062 & 0.289185744 \\
\hline 13 & 0.008688839 & 7.54959E-05 & 0.007287 & 0.023656 & 0.002725 & 0.373944707 \\
\hline 14 & 0.003252449 & 1.05784E-05 & 0.006883 & 0.026908 & 0.002778 & 0.403526657 \\
\hline 15 & -0.000758673 & 5.75584E-07 & 0.006271 & 0.02615 & 0.002424 & 0.386546181 \\
\hline 17 & -0.010510085 & 0.000110462 & 0.006695 & 0.01564 & 0.001131 & 0.168872615 \\
\hline 18 & 0.004907278 & $2.40814 \mathrm{E}-05$ & 0.006516 & 0.020547 & 0.001508 & 0.231465725 \\
\hline 21 & 2.58642E-05 & $6.68958 \mathrm{E}-10$ & 0.005867 & 0.020573 & 0.00136 & 0.231804528 \\
\hline 22 & 0.01500901 & 0.00022527 & 0.006781 & 0.035582 & 0.002725 & 0.401831348 \\
\hline 23 & 0.00122764 & $1.5071 \mathrm{E}-06$ & 0.006226 & 0.036809 & 0.002575 & 0.413626313 \\
\hline 24 & -0.001144271 & 1.30936E-06 & 0.005718 & 0.035665 & 0.002203 & 0.385335638 \\
\hline 25 & -0.012399371 & 0.000153744 & 0.006386 & 0.023266 & 0.000743 & \begin{tabular}{|l|l|}
0.116345884 \\
\end{tabular} \\
\hline 27 & 0.018904852 & 0.000357393 & 0.007638 & 0.042171 & 0.002559 & 0.335066637 \\
\hline 28 & 0.001225708 & 1.50236E-06 & 0.006997 & 0.043396 & 0.002426 & 0.346715445 \\
\hline 29 & -0.002 & $4 \mathrm{E}-06$ & 548 & 0.04 & 0.00 & 0.295136281 \\
\hline 31 & 0.007195508 & 5.17753E-05 & 0.006612 & 0.048084 & 0.002459 & 0.371837808 \\
\hline 33 & 0.000265979 & $7.0745 \mathrm{E}-08$ & 0.005978 & 0.04835 & 0.002239 & 0.374632784 \\
\hline 34 & 0.013920721 & 0.000193786 & 0.006772 & 0.062271 & 0.003408 & 0.503183251 \\
\hline 35 & 0.000977714 & 9.55924E-07 & 0.006193 & 0.063249 & 0.003165 & 0.511027127 \\
\hline 36 & -0.001696866 & 2.87935E-06 & 0.005743 & 0.061552 & 0.002678 & 0.466381905 \\
\hline 37 & -0.008614462 & 7.4209E-05 & 0.00603 & 0.052937 & 0.001549 & 0.256902056 \\
\hline 38 & -0.006469709 & 4.18571E-05 & 0.006074 & 0.046468 & 0.000747 & 0.123027072 \\
\hline 40 & -0.001560658 & 2.43565E-06 & 0.005623 & 0.044907 & 0.000516 & 0.091856547 \\
\hline 42 & 0.000777524 & 6.04543E-07 & 0.005138 & 0.045684 & 0.000543 & 0.105598544 \\
\hline 43 & 0.005106622 & 2.60776E-05 & 0.005135 & 0.050791 & 0.000999 & 0.194542325 \\
\hline 44 & 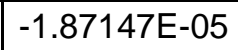 & $3.5024 \mathrm{E}-10$ & 0.004623 & 0.050772 & 0.000897 & 0.194058804 \\
\hline 45 & -0.003249453 & 1.05589E-05 & 0.004486 & 0.047523 & 0.000483 & 0.107568147 \\
\hline 46 & -0.00563538 & 3.17575E-05 & 0.004601 & 0.041887 & -0.00013 & -0.028088393 \\
\hline \multirow[t]{4}{*}{48} & 0.014090234 & 0.000198535 & 0.00555 & 0.055978 & 0.001293 & 0.232924021 \\
\hline & SSE & 0.002318345 & & & & \\
\hline & SSE/35 & 6.62384E-05 & & & & \\
\hline & DELTA(0) & 0.00694326 & & & & \\
\hline
\end{tabular}

Table A.3.2 Tracking Signal Analysis\{ARMA(1,1) process, 11 observations missing\} 


\begin{tabular}{|c|c|c|c|c|c|c|}
\hline Period & Error & Error Sq. & Delta(T) & $\mathrm{Y}(\mathrm{T})$ & $Q(T)$ & $\mathrm{Q}(\mathrm{T}) / \operatorname{Delta}(\mathrm{T})$ \\
\hline 1 & -0.01127 & 0.000126927 & 0.008468 & -0.01127 & -0.00113 & -0.133041823 \\
\hline 2 & -0.00977 & 9.55042E-05 & 0.008599 & -0.02104 & -0.00199 & -0.231574836 \\
\hline 3 & -0.00847 & 7.18104E-05 & 0.008586 & -0.02951 & -0.00264 & -0.307414605 \\
\hline 4 & -0.00962 & 9.26307E-05 & 0.00869 & -0.03914 & -0.00334 & -0.384121073 \\
\hline 5 & -0.00634 & 4.0192E-05 & 0.008455 & -0.04548 & -0.00364 & -0.430301079 \\
\hline 7 & 0.011834 & 0.000140044 & 0.008793 & -0.03364 & -0.00209 & -0.237801584 \\
\hline 8 & 0.00456 & 2.07915E-05 & 0.00837 & -0.02908 & -0.00143 & -0.170365514 \\
\hline 9 & -0.00186 & 3.47711E-06 & 0.007719 & -0.03095 & -0.00147 & -0.190407072 \\
\hline 10 & -0.00559 & $3.1216 \mathrm{E}-05$ & 0.007506 & -0.03654 & -0.00188 & -0.250670516 \\
\hline 12 & 0.011502 & 0.000132303 & 0.007906 & -0.02503 & -0.00054 & -0.068701724 \\
\hline 13 & 0.004689 & 2.19852E-05 & 0.007584 & -0.02034 & $-2 E-05$ & -0.002627468 \\
\hline 14 & -0.00075 & 5.58832E-07 & 0.0069 & -0.02109 & $-9.3 E-05$ & -0.013432734 \\
\hline 15 & -0.00476 & $2.2645 \mathrm{E}-05$ & 0.006686 & -0.02585 & -0.00056 & -0.083649708 \\
\hline 17 & 0.00651 & 4.23812E-05 & 0.006668 & -0.01934 & 0.000148 & 0.022141543 \\
\hline 18 & 0.000907 & 8.23154E-07 & 0.006092 & -0.01843 & 0.000224 & 0.03670391 \\
\hline 21 & 0.021904 & 0.000479794 & 0.007674 & 0.003471 & 0.002392 & 0.311678142 \\
\hline 22 & 0.011009 & 0.000121198 & 0.008007 & 0.01448 & 0.003253 & 0.40631617 \\
\hline 23 & -0.00277 & 7.68598E-06 & 0.007484 & 0.011708 & 0.002651 & 0.354218107 \\
\hline 24 & -0.00514 & 2.64635E-05 & 0.00725 & 0.006564 & 0.001871 & 0.258124618 \\
\hline 26 & 0.009095 & 8.27175E-05 & 0.007434 & 0.015659 & 0.002594 & 0.348884843 \\
\hline 27 & 0.001104 & 1.21956E-06 & 0.006801 & 0.016763 & 0.002445 & 0.359457229 \\
\hline 28 & -0.00998 & 9.95247E-05 & 0.007119 & 0.006787 & 0.001203 & 0.168942081 \\
\hline 29 & -0.0121 & 0.000146482 & 0.007617 & -0.00532 & -0.00013 & -0.016792562 \\
\hline 31 & 0.001694 & 2.87001E-06 & 0.007025 & -0.0 & 5.43E-05 & 0.007728411 \\
\hline 33 & 0.018536 & 0.000343581 & 0.008176 & 0.014914 & 0.001902 & 0.232689385 \\
\hline 35 & 0.022462 & 0.000504522 & 0.009605 & 0.037375 & 0.003958 & 0.412136378 \\
\hline 36 & 0.010594 & 0.000112223 & 0.009703 & 0.047969 & 0.004622 & 0.476315502 \\
\hline 37 & -0.00589 & 3.47126E-05 & 0.009322 & 0.042077 & 0.003571 & 0.383011012 \\
\hline 38 & -0.00644 & 4.15201E-05 & 0.009034 & 0.035634 & 0.002569 & 0.284370272 \\
\hline 40 & 0.001341 & 1.79765E-06 & 0.008265 & 0.036974 & 0.002446 & 0.295979332 \\
\hline 42 & -0.00344 & 1.18482E-05 & 0.007783 & 0.033532 & 0.001857 & 0.238661125 \\
\hline 43 & 0.001539 & 2.36707E-06 & 0.007158 & 0.035071 & 0.001826 & 0.255024513 \\
\hline 44 & -0.00489 & 2.39534E-05 & 0.006932 & 0.030177 & 0.001154 & 0.166414379 \\
\hline 45 & -0.00823 & 6.76551E-05 & 0.007061 & 0.021951 & 0.000216 & 0.030544813 \\
\hline \multirow[t]{4}{*}{46} & -0.00785 & 6.16447E-05 & 0.00714 & 0.0141 & -0.00059 & -0.082773778 \\
\hline & SSE & 0.003017069 & & & & \\
\hline & SSE/33 & 9.14263E-05 & & & & \\
\hline & $\operatorname{DELTA}(0)$ & 0.008157256 & & & & \\
\hline
\end{tabular}

Table A.3.3 Tracking Signal Analysis\{ARMA(1,1) process, 13 observations missing\} 


\begin{tabular}{|c|c|c|c|c|c|c|}
\hline Period & Error & Error Sq. & Delta(T) & $\mathrm{Y}(\mathrm{T})$ & $\mathrm{Q}(\mathrm{T})$ & $\mathrm{Q}(\mathrm{T}) / \operatorname{Delta}(\mathrm{T})$ \\
\hline 1 & -0.00127 & 1.60321E-06 & 0.007187 & -0.00127 & -0.00013 & -0.017617064 \\
\hline 2 & -0.01677 & 0.000281321 & 0.008146 & -0.01804 & -0.00179 & -0.21989561 \\
\hline 4 & 0.007533 & 5.67429E-05 & 0.008084 & -0.01051 & -0.00086 & -0.106230602 \\
\hline 5 & -0.00051 & 2.60276E-07 & 0.007327 & -0.01102 & -0.00082 & -0.112453801 \\
\hline 7 & 0.011022 & 0.000121491 & \begin{tabular}{|l|}
0.007697 \\
\end{tabular} & $6.1 \mathrm{E}-06$ & 0.000361 & 0.046861254 \\
\hline 8 & 0.00083 & $6.8964 \mathrm{E}-07$ & 0.00701 & 0.000837 & 0.000408 & 0.058152773 \\
\hline 9 & -0.00748 & 5.58818E-05 & 0.007056 & -0.00664 & -0.00038 & -0.053944353 \\
\hline 12 & 0.02123 & 0.000450721 & 0.008474 & 0.014591 & 0.00178 & 0.210108016 \\
\hline 13 & 0.002282 & 5.20906E-06 & 0.007855 & 0.016874 & 0.001831 & 0.23305983 \\
\hline 14 & -0.00581 & 3.3728E-05 & 0.00765 & 0.011066 & 0.001067 & 0.139450754 \\
\hline 15 & -0.01165 & 0.000135729 & 0.00805 & -0.00058 & -0.0002 & -0.025454597 \\
\hline 18 & 0.002059 & 4.23958E-06 & 0.007451 & 0.001475 & $2.15 \mathrm{E}-05$ & 0.002883273 \\
\hline 21 & 0.014609 & 0.000213432 & 0.008167 & 0.016084 & 0.00148 & 0.1812547 \\
\hline 22 & -0.00252 & 6.36588E-06 & 0.007602 & 0.013561 & 0.00108 & 0.142051446 \\
\hline 23 & -0.00981 & 9.61555E-05 & 0.007823 & 0.003755 & -8.7E-06 & -0.001105872 \\
\hline 25 & -0.00394 & 1.55383E-05 & 0.007435 & -0.00019 & -0.0004 & -0.05406729 \\
\hline 26 & 0.000879 & 7.72547E-07 & 0.006779 & 0.000692 & -0.00027 & -0.040400717 \\
\hline 27 & -0.00643 & 4.12865E-05 & 0.006744 & -0.00573 & -0.00089 & -0.131831901 \\
\hline 28 & -0.02173 & 0.000472024 & 0.008242 & -0.02746 & -0.00297 & -0.360684143 \\
\hline 31 & -0.00098 & 9.65373E-07 & 0.007516 & -0.02844 & -0.00277 & -0.369041619 \\
\hline 33 & 0.012473 & 0.000155585 & 0.008012 & -0.01597 & -0.00125 & -0.155897701 \\
\hline 34 & 0.000461 & 2.12454E-07 & 0.007257 & -0.01551 & -0.00108 & -0.148555694 \\
\hline 35 & -0.00882 & 7.78617E-05 & 0.007413 & -0.02433 & -0.00185 & -0.249900337 \\
\hline 37 & 0.003468 & 1.20299E-05 & 0.007019 & -0.02086 & -0.00132 & -0.188136102 \\
\hline 38 & -0.00079 & 6.26232E-07 & 0.006396 & -0.02165 & -0.00127 & -0.1981807 \\
\hline 40 & -0.00374 & 1.39653E-05 & 0.00613 & -0.02539 & -0.00151 & -0.247059896 \\
\hline 43 & 0.009061 & 8.20993E-05 & 0.006423 & -0.01633 & -0.00046 & -0.071146379 \\
\hline 44 & -0.00511 & 2.60753E-05 & 0.006292 & -0.02144 & -0.00092 & -0.146534049 \\
\hline 46 & 0.002272 & 5.16257E-06 & 0.00589 & -0.01916 & -0.0006 & -0.102302719 \\
\hline \multirow[t]{4}{*}{48} & 3.47E-05 & 1.20468E-09 & 0.005304 & -0.01913 & -0.00054 & -0.101581411 \\
\hline & SSE & 0.002367776 & & & & \\
\hline & SSE/28 & 8.45634E-05 & & & & \\
\hline & $\operatorname{DELTA}(0)$ & 0.007845121 & & & & \\
\hline
\end{tabular}

Table A.3.4 Tracking Signal Analysis\{ARMA(1,1) process, 18 observations missing\} 


\begin{tabular}{|c|c|c|c|c|c|c|}
\hline Period & Error & Error Sq. & Delta(T) & $\mathrm{Y}(\mathrm{T})$ & $\mathrm{Q}(\mathrm{T})$ & $\mathrm{Q}(\mathrm{T}) / \operatorname{Delta}(\mathrm{T})$ \\
\hline 1 & -0.003371379 & 1.13662E-05 & 0.004268 & -0.00337 & -0.000337138 & -0.07898444 \\
\hline 2 & 0.002124533 & 4.51364E-06 & 0.004054 & -0.00125 & -9.09708E-05 & -0.022439652 \\
\hline 3 & -0.00172442 & 2.97362E-06 & 0.003821 & -0.00297 & -0.000254316 & -0.066556304 \\
\hline 4 & 0.002672247 & $7.1409 \mathrm{E}-06$ & 0.003706 & -0.0003 & 3.83405E-05 & 0.010345026 \\
\hline 5 & -0.001439163 & 2.07119E-06 & 0.003479 & -0.00174 & -0.00010941 & -0.031444311 \\
\hline 7 & 0.010116298 & 0.000102339 & 0.004143 & 0.008378 & 0.000913161 & 0.220402032 \\
\hline 8 & 0.003241692 & 1.05086E-05 & 0.004053 & 0.01162 & 0.001146014 & 0.282756048 \\
\hline 9 & 0.002430894 & 5.90925E-06 & 0.003891 & 0.014051 & 0.001274502 & 0.327567996 \\
\hline 11 & 0.000115847 & 1.34205E-08 & 0.003513 & 0.014167 & 0.001158637 & 0.329 \\
\hline 12 & 0.001259603 & $1.5866 \mathrm{E}-06$ & 0.003288 & 0.015426 & 0.001168733 & 0.355461072 \\
\hline 13 & 0.002152153 & 4.63176E-06 & 0.003174 & 0.017578 & 0.001267075 & 0.399159568 \\
\hline 15 & 0.000156342 & 2.44429E-08 & 0.002873 & 0.017735 & 0.001156002 & 0.402429715 \\
\hline 17 & 9.85123E-05 & 7E-09 & 2595 & 0.01 & 0.001050253 & 0.40 \\
\hline 18 & -0.000623901 & 3.89252E-07 & 0.002398 & 0.017209 & 0.000882838 & 0.368151717 \\
\hline 20 & 0.00517085 & 2.67377E-05 & 0.002675 & 0.02238 & 0.001311639 & 0.490275665 \\
\hline 21 & -0.002764253 & E-06 & 2684 & 616 & 0.000 & 0.336 \\
\hline 22 & -0.000552734 & 3.05515E-07 & 0.002471 & 0.019063 & 0.000758371 & 0.306901635 \\
\hline 23 & -0.008803789 & 7.75067E-05 & 0.003104 & 0.010259 & -0.000197845 & -0.06373186 \\
\hline 25 & 0.000691678 & 4.78418E-07 & 0.002863 & 0.010951 & -0.000108892 & -0.0380 \\
\hline 26 & -0.002275442 & 5.17764E-06 & 0.002804 & 0.008676 & -0.000325547 & -0.116088573 \\
\hline 27 & -0.009829437 & 9.66178E-05 & 0.003507 & -0.00115 & -0.001275936 & -0.363844715 \\
\hline 29 & 0.006256252 & 3.91407E-05 & 0.003782 & 0.005102 & -0.000522717 & -0.138220715 \\
\hline 30 & 0.001202789 & 1.4467E-06 & 0.003524 & 0.006305 & -0.000350167 & -0.099370196 \\
\hline 31 & -0.004519619 & 2.0427E-05 & 0.003623 & 0.001786 & -0.000767112 & -0.211708357 \\
\hline 32 & -0.002618157 & 6.85475E-06 & 0.003523 & -0.00083 & -0.000952217 & -0.270292641 \\
\hline 33 & -0.007021941 & 7E-05 & 873 & -0.00785 & -0.001559189 & -0.402598585 \\
\hline 35 & 0.003675521 & 1.35095E-05 & 0.003853 & -0.00418 & -0.001035718 & -0.268802377 \\
\hline 36 & -0.0036781 & 1.35284E-05 & 0.003836 & -0.00786 & -0.001299956 & -0.338919909 \\
\hline 37 & -0.001091831 & 1.19209E-06 & 0.003561 & -0.00895 & -0.001279144 & -0.359187949 \\
\hline 38 & -0.010740062 & 0.000115349 & 0.004279 & -0.01969 & -0.002225236 & -0.520024792 \\
\hline 39 & -0.003368859 & 1.13492E-05 & 0.004188 & -0.02306 & -0.002339598 & -0.558633704 \\
\hline 41 & -0.008030842 & 6.44944E-05 & 0.004572 & -0.03109 & -0.002908722 & -0.636154982 \\
\hline 43 & 0.005038209 & 2.53835E-05 & 0.004619 & -0.02605 & -0.002114029 & -0.457687632 \\
\hline 45 & 0.000921287 & 8.4877E-07 & 0.004249 & -0.02513 & -0.001810498 & -0.426082663 \\
\hline 46 & 0.002544335 & 6.47364E-06 & 0.004079 & -0.02258 & -0.001375014 & -0.337121851 \\
\hline 47 & -0.002975391 & 8.85295E-06 & 0.003968 & -0.02556 & -0.001535052 & -0.386823065 \\
\hline \multirow[t]{4}{*}{48} & -0.013094056 & 0.000171454 & 0.004881 & -0.03865 & -0.002690952 & -0.551319962 \\
\hline & SSE & 0.000917555 & & & & \\
\hline & SSE/35 & 2.62159E-05 & & & & \\
\hline & Delta $(0)$ & 0.00436808 & & & & \\
\hline
\end{tabular}

Table A.4.1 Tracking Signal Analysis \{ARMA(1,2) process, 11 observations missing\} 


\begin{tabular}{|c|c|c|c|c|c|c|}
\hline Period & Error & Error Sq. & Delta(T) & $Y(T)$ & $\mathrm{Q}(\mathrm{T})$ & $\mathrm{Q}(\mathrm{T}) / \operatorname{Delta}(\mathrm{T})$ \\
\hline 1 & -0.00337 & $1.13662 \mathrm{E}-05$ & 0.004405 & -0.00337 & -0.000337138 & -0.076538032 \\
\hline 2 & 0.002125 & 4.51364E-06 & 0.004177 & -0.00125 & $-9.09708 \mathrm{E}-05$ & -0.021779975 \\
\hline 3 & -0.00172 & 2.97362E-06 & 0.003932 & -0.00297 & -0.000254316 & -0.064685512 \\
\hline 4 & 0.002672 & 7.1409E-06 & 0.003806 & -0.0003 & 3.83405E-05 & 0.010074662 \\
\hline 5 & -0.00144 & 2.07119E-06 & 0.003569 & -0.00174 & -0.00010941 & -0.030655661 \\
\hline 7 & 0.007116 & 5.06417E-05 & 0.003924 & 0.005378 & 0.000613161 & 0.156270224 \\
\hline 8 & 0.001742 & 3.03349E-06 & 0.003706 & 0.00712 & 0.000726014 & 0.19592776 \\
\hline 9 & 0.000931 & 8.66564E-07 & 0.003428 & 0.008051 & 0.000746502 & 0.217762463 \\
\hline 11 & 0.009742 & 9.48993E-05 & 0.004059 & 0.017792 & 0.001646015 & 0.405480847 \\
\hline 12 & -0.00024 & 5.77908E-08 & 0.003678 & 0.017552 & 0.001457373 & 0.396293289 \\
\hline 13 & 0.000652 & $4.25304 \mathrm{E}-07$ & 0.003375 & 0.018204 & 0.001376851 & 0.407958831 \\
\hline 15 & 0.000646 & 4.17179E-07 & 0.003102 & 0.01885 & 0.001303756 & 0.420285959 \\
\hline 17 & 0.00235 & 5.52191E-06 & 0.003027 & 0.0212 & 0.001408368 & 0.465291694 \\
\hline 18 & -0.00212 & 4.51095E-06 & 0.002937 & 0.019076 & 0.001055141 & 0.359312579 \\
\hline 20 & 0.003671 & 1.34751E-05 & 0.00301 & 0.022747 & 0.001316712 & 0.437448143 \\
\hline 21 & -0.00426 & 1.81839E-05 & 0.003135 & 0.018483 & 0.000758615 & 0.241950847 \\
\hline 22 & -0.00205 & 4.21372E-06 & 0.003027 & 0.01643 & 0.00047748 & 0.157732982 \\
\hline 23 & .0103 & 0.000106168 & 0.003 & 26 & -0.00060 & -0.15996 \\
\hline 25 & 0081 & 385E-07 & 0.00346 & 0.005318 & -0.000621414 & -0.179591216 \\
\hline 27 & 0.001367 & $1.86855 \mathrm{E}-06$ & 0.003251 & 0.006685 & -0.000422578 & -0.129990394 \\
\hline 29 & 0.009632 & 9.27794E-05 & 0.003889 & 0.016317 & 0.0005829 & 0.149885386 \\
\hline 30 & 0.002504 & 6.26988E-06 & 0.00375 & 0.018821 & 0.000775008 & 0.206642555 \\
\hline 31 & 494 & 311E-05 & 0.0 & 0.0 & 0.0 & 0.052517852 \\
\hline 33 & $0<4 \pi$ & 6.09934E-06 & 0.00373 & 0.016348 & 0.000429874 & 0.115256933 \\
\hline 35 & 0.005899 & 3.48004E-05 & 0.003947 & 0.022247 & 0.000976806 & 0.247502353 \\
\hline 36 & -0.00383 & 1.46811E-05 & 0.003935 & 0.018415 & 0.000495965 & 0.126034777 \\
\hline 38 & -0.00442 & 1.95357E-05 & 0.003984 & 0.013995 & 4.37695E-06 & 0.001098736 \\
\hline 39 & -0.00348 & 1.21286E-05 & 0.003934 & 0.010513 & -0.000344322 & -0.087535247 \\
\hline 41 & -0.00911 & 8.30113E-05 & 0.004451 & 0.001402 & -0.001220995 & -0.274302298 \\
\hline 43 & 0.002698 & 7.27932E-06 & 0.004276 & 0.0041 & -0.000829093 & -0.193896879 \\
\hline 46 & 0.002866 & 8.21365E-06 & 0.004135 & 0.006966 & -0.000459589 & -0.111147518 \\
\hline 47 & -0.00422 & 1.77705E-05 & 0.004143 & 0.00275 & -0.000835181 & -0.201588283 \\
\hline \multirow[t]{4}{*}{48} & -0.01449 & 0.000210076 & 0.005178 & -0.01174 & -0.002201063 & -0.425071164 \\
\hline & SSE & 0.000870079 & & & & \\
\hline & SSE/31 & 2.80671E-05 & & & & \\
\hline & Delta(0) & 0.004519671 & & & & \\
\hline
\end{tabular}

Table A.4.2 Tracking Signal Analysis\{ARMA(1,2) process, 15 observations missing\} 


\begin{tabular}{|c|c|c|c|c|c|c|}
\hline Period & Error & Error Sq. & Delta(T) & $Y(T)$ & $\mathrm{Q}(\mathrm{T})$ & $\mathrm{Q}(\mathrm{T}) / \operatorname{Delta}(\mathrm{T})$ \\
\hline 1 & -0.00337 & 1.13662E-05 & 0.005226 & -0.00337 & -0.00034 & -0.064506021 \\
\hline 2 & -0.00288 & $8.26831 \mathrm{E}-06$ & 0.004991 & -0.00625 & -0.00059 & -0.118398812 \\
\hline 3 & -0.00172 & 2.97362E-06 & 0.004665 & -0.00797 & -0.0007 & -0.150989597 \\
\hline 4 & 0.002672 & $7.1409 \mathrm{E}-06$ & 0.004465 & -0.0053 & -0.00037 & -0.082110815 \\
\hline 5 & -0.00144 & 2.07119E-06 & 0.004163 & -0.00674 & -0.00047 & -0.113844099 \\
\hline 7 & 0.007116 & 5.06417E-05 & 0.004458 & 0.000378 & 0.000285 & 0.063952806 \\
\hline 8 & 0.001742 & 3.03349E-06 & 0.004187 & 0.00212 & 0.000431 & 0.102894781 \\
\hline 9 & 0.000931 & 8.66564E-07 & 0.003861 & 0.003051 & 0.000481 & 0.124524482 \\
\hline 11 & 0.009742 & 9.48993E-05 & 0.004449 & 0.012792 & 0.001407 & 0.316220106 \\
\hline 12 & -0.00024 & 5.77908E-08 & 0.004028 & 0.012552 & 0.001242 & 0.308364994 \\
\hline 13 & 0.000652 & 4.25304E-07 & 0.003691 & 0.013204 & 0.001183 & 0.320586809 \\
\hline 15 & 0.000785 & $6.15471 \mathrm{E}-07$ & 0.0034 & 0.013989 & 0.001143 & 0.336263913 \\
\hline 17 & 0.011867 & 0.000140831 & 0.004247 & 0.025856 & 0.002216 & 0.521743104 \\
\hline 18 & -0.00212 & 4.51095E-06 & 0.004034 & 0.023732 & 0.001782 & 0.441631176 \\
\hline 20 & 0.003671 & $1.34751 \mathrm{E}-05$ & 0.003998 & 0.027403 & 0.001971 & 0.492898508 \\
\hline 21 & -0.00426 & 1.81839E-05 & 0.004025 & 0.023139 & 0.001347 & 0.334721289 \\
\hline 22 & -0.00205 & 4.21372E-06 & 0.003827 & 0.021086 & 0.001007 & 0.263137992 \\
\hline 23 & -0.0103 & 0.000106168 & 0.004475 & 0.010782 & -0.00012 & -0.027695846 \\
\hline 27 & 0.007425 & $5.5128 \mathrm{E}-05$ & 0.00477 & 0.018207 & 0.000631 & 0.132269383 \\
\hline 29 & 0.011751 & 0.000138086 & 0.005468 & 0.029958 & 0.001743 & 0.318743493 \\
\hline 31 & 0.00405 & 1.64012E-05 & 0.005326 & 0.034008 & 0.001974 & 0.370542334 \\
\hline 33 & 0.00637 & 4.0583E-05 & 0.005431 & 0.040378 & 0.002413 & 0.444380138 \\
\hline 35 & 0.006684 & 4.46808E-05 & 0.005556 & 0.047063 & 0.00284 & 0.51122492 \\
\hline 36 & -0.00394 & $1.55011 \mathrm{E}-05$ & 0.005394 & 0.043125 & 0.002163 & 0.400923301 \\
\hline 38 & -0.00451 & 2.03074E-05 & 0.005305 & 0.038619 & 0.001496 & 0.281930513 \\
\hline 39 & -0.00492 & 2.41887E-05 & 0.005267 & 0.033701 & 0.000854 & 0.162220151 \\
\hline 41 & -0.00982 & 9.64001E-05 & 0.005722 & 0.023882 & -0.00021 & -0.037209161 \\
\hline 43 & 0.000707 & 4.99894E-07 & 0.00522 & 0.024589 & -0.00012 & -0.023161551 \\
\hline 46 & 0.002677 & 7.16885E-06 & 0.004966 & 0.027267 & 0.000159 & 0.032002225 \\
\hline 47 & -0.00484 & 2.34126E-05 & 0.004953 & 0.022428 & -0.00034 & -0.068808385 \\
\hline \multirow[t]{4}{*}{48} & -0.01496 & 0.000223861 & 0.005954 & 0.007466 & -0.0018 & -0.302801828 \\
\hline & SSE & 0.001175961 & & & & \\
\hline & SSE/29 & 4.05504E-05 & & & & \\
\hline & $\operatorname{Delta}(0)$ & 0.005432577 & & & & \\
\hline
\end{tabular}

Table A.4.3 Tracking Signal Analysis \{ARMA(1,2) process, 17 observations missing\} 


\begin{tabular}{|c|c|c|c|c|c|c|}
\hline Period & Error & Error Sq. & Delta(T) & $Y(T)$ & $Q(T)$ & $\mathrm{Q}(\mathrm{T}) / \operatorname{Delta}(\mathrm{T})$ \\
\hline 1 & -0.00337 & 1.13662E-05 & 0.005512 & -0.00337 & -0.00034 & -0.061165489 \\
\hline 2 & 0488 & 2.37702E-05 & 0.005448 & 825 & -0.0 & -0.145178752 \\
\hline 3 & -0.00872 & 7.61155E-05 & 0.005776 & -0.01697 & -0.00158 & -0.274299001 \\
\hline 4 & -0.00433 & 1.87294E-05 & 0.005631 & -0.0213 & -0.00186 & -0.33007278 \\
\hline 5 & -0.00844 & 7.12195E-05 & 0.005912 & -0.02974 & -0.00252 & -0.425704535 \\
\hline 7 & 0.008116 & 6.58743E-05 & 0.006132 & -0.02162 & -0.00145 & -0.237008303 \\
\hline 8 & 0.002742 & 7.51688E-06 & 0.005793 & -0.01888 & -0.00103 & -0.178466106 \\
\hline 9 & 0.001931 & 3.72835E-06 & 0.005407 & -0.01695 & -0.00074 & -0.136382014 \\
\hline 11 & 0.010742 & 0.000115383 & 0.00594 & -0.00621 & 0.00041 & 0.069099686 \\
\hline 12 & 0.00076 & 5.76997E-07 & 0.005422 & -0.00545 & 0.000445 & 0.082140332 \\
\hline 13 & 0.001652 & $2.72961 \mathrm{E}-06$ & 0.005045 & -0.0038 & 0.000566 & 0.112196527 \\
\hline 15 & 0.00838 & 7.02231E-05 & 0.005379 & 0.004584 & 0.001347 & 0.250511693 \\
\hline 17 & 0.012867 & 0.000165565 & 0.006128 & 0.017451 & 0.002499 & 0.407893469 \\
\hline 18 & -0.00112 & $1.26315 \mathrm{E}-06$ & 0.005627 & 0.016327 & 0.002137 & 0.379774532 \\
\hline 20 & 0.004671 & 2.18168E-05 & 0.005532 & 0.020998 & 0.00239 & 0.432145623 \\
\hline 21 & -0.00326 & 1.06553E-05 & 0.005305 & 0.017734 & 0.001825 & 0.344021727 \\
\hline 22 & -0.00105 & 1.10825E-06 & 0.00488 & 0.016681 & 0.001537 & 0.315026046 \\
\hline 23 & -0.0093 & 8.65605E-05 & 0.005322 & 0.007377 & 0.000453 & 0.085140563 \\
\hline 27 & 0.008425 & 05 & 0.0 & 302 & 25 & 0.221984022 \\
\hline 29 & 0.012751 & 0.000162588 & 0.006344 & 0.028553 & 0.0024 & 0.378354291 \\
\hline 31 & 0.00505 & 2.55009E-05 & 0.006215 & 0.033603 & 0.002665 & 0.428866203 \\
\hline 33 & 0.00737 & 5.43239E-05 & 0.00633 & 0.040974 & 0.003136 & 0.495363642 \\
\hline 35 & 0.007684 & 5.90496E-05 & 0.006466 & 0.048658 & 0.003591 & 0.555338219 \\
\hline 36 & -0.00294 & 8.6268E-06 & 0.006113 & 0.045721 & 0.002938 & 0.480606964 \\
\hline 38 & -0.00351 & 1.22946E-05 & 0.005852 & 0.042214 & 0.002293 & 0.391896508 \\
\hline 39 & -0.00392 & 1.53523E-05 & 0.005659 & 0.038296 & 0.001672 & 0.295521114 \\
\hline 41 & -0.00882 & 7.77634E-05 & 0.005975 & 0.029478 & 0.000623 & 0.104311781 \\
\hline 43 & 0.001707 & 2.91396E-06 & 0.005548 & 0.031185 & 0.000732 & 0.1318706 \\
\hline 46 & 0.003677 & 1.35238E-05 & 0.005361 & 0.034862 & 0.001026 & 0.191421862 \\
\hline \multirow[t]{4}{*}{47} & -0.00384 & 1.47353E-05 & 0.005209 & 0.031024 & 0.00054 & 0.103618173 \\
\hline & SSE & 0.001271852 & & & & \\
\hline & SSE/28 & 4.54233E-05 & & & & \\
\hline & Delta(0) & 0.005749733 & & & & \\
\hline
\end{tabular}

Table A.4.4 Tracking Signal Analysis $\{\operatorname{ARMA}(1,2)$ process, 18 observations missing $\}$ 


\begin{tabular}{|c|c|c|c|c|c|c|}
\hline Period & Error & Error Sq. & $\operatorname{DELTA}(\mathrm{T})$ & $Y(T)$ & $\mathrm{Q}(\mathrm{T})$ & $\mathrm{Q}(\mathrm{T}) / \operatorname{Delta}(\mathrm{T})$ \\
\hline 1 & -0.0003 & $9.18865 E-08$ & 0.005426 & -0.0003 & $-3.03128 E-05$ & -0.005586531 \\
\hline 3 & 0.009729 & 9.46617E-05 & 0.005856 & 0.009426 & 0.000945661 & 0.161475175 \\
\hline 4 & -0.0008 & 6.44469E-07 & 0.005351 & 0.008624 & 0.000770816 & 0.144050132 \\
\hline 5 & $\mid-0.00396$ & 1.56969E-05 & 0.005212 & 0.004662 & 0.000297541 & 0.057086351 \\
\hline 8 & 0.011562 & 0.000133688 & 0.005847 & 0.016224 & 0.00142402 & 0.243541395 \\
\hline 9 & 0.002735 & 7.48095E-06 & 0.005536 & 0.018959 & 0.001555132 & 0.280915634 \\
\hline 10 & -0.00239 & 5.72966E-06 & 0.005222 & 0.016565 & 0.001160251 & 0.222197512 \\
\hline 11 & -0.00276 & 7.61247E-06 & 0.004975 & 0.013806 & 0.000768319 & 0.154422101 \\
\hline 14 & 0.007934 & 6.29559E-05 & 0.005271 & 0.021741 & 0.001484935 & 0.281699155 \\
\hline 15 & -0.00324 & $1.05075 E-05$ & 0.005068 & 0.018499 & 0.001012288 & 0.199726618 \\
\hline 16 & $-1.4 \mathrm{E}-05$ & $1.84194 \mathrm{E}-10$ & 0.004563 & 0.018486 & 0.000909702 & 0.199369773 \\
\hline 18 & 0.004961 & 2.46112E-05 & 0.004603 & 0.023447 & 0.001314829 & 0.285664854 \\
\hline 19 & -0.00137 & 1.8692E-06 & 0.004279 & 0.022079 & 0.001046627 & 0.244587859 \\
\hline 21 & 0.005464 & 2.98553E-05 & 0.004398 & 0.027543 & 0.001488364 & 0.338446819 \\
\hline 22 & 0.000248 & 6.1297E-08 & 0.003983 & 0.027791 & 0.001364286 & 0.342559402 \\
\hline 23 & -0.00736 & 5.41073E-05 & 0.00432 & 0.020435 & 0.000492281 & 0.113955553 \\
\hline 24 & -0.00682 & 4.64962E-05 & 0.00457 & 0.013616 & -0.000238828 & -0.052261978 \\
\hline 25 & -0.00981 & 9.62816E-05 & 0.005094 & 0.003804 & -0.001196177 & -0.234817318 \\
\hline 29 & 0.017984 & 0.000323426 & 0.006383 & 0.021788 & 0.000721846 & 0.113087553 \\
\hline 30 & -0.00376 & 1.41075E-05 & 0.00612 & 0.018032 & 0.000274062 & 0.04477872 \\
\hline 32 & 0.005297 & 2.80607E-05 & 0.006038 & 0.023329 & 0.00077638 & 0.128581104 \\
\hline 35 & 0.009379 & 8.79639E-05 & 0.006372 & 0.032708 & 0.001636632 & 0.256841898 \\
\hline 37 & 0.002712 & 7.35626E-06 & 0.006006 & 0.035421 & 0.001744193 & 0.290401257 \\
\hline 38 & -0.00271 & 7.36679E-06 & 0.005677 & 0.032706 & 0.001298356 & 0.228706441 \\
\hline 39 & -0.00598 & 3.57542E-05 & 0.005707 & 0.026727 & 0.000570572 & 0.099973939 \\
\hline 40 & -0.0088 & 7.73595E-05 & 0.006016 & 0.017931 & -0.000366028 & -0.060842153 \\
\hline 42 & -0.00676 & 4.57091E-05 & 0.006091 & 0.011171 & -0.00100551 & -0.165094585 \\
\hline 45 & 0.009821 & 9.6455E-05 & 0.006464 & 0.020992 & 7.71557E-05 & 0.011937005 \\
\hline 46 & 0.004361 & $1.90216 \mathrm{E}-05$ & 0.006253 & 0.025353 & 0.000505578 & 0.080849018 \\
\hline \multirow[t]{4}{*}{48} & 0.006918 & 4.78613E-05 & 0.00632 & 0.032271 & 0.001146839 & 0.181466473 \\
\hline & SSE & 0.001382794 & & & & \\
\hline & SSE/28 & 4.93855E-05 & & & & \\
\hline & $\operatorname{DELTA}(0)$ & 0.005995261 & & & & \\
\hline
\end{tabular}

Table A.5.1 Tracking Signal Analysis $\{\operatorname{ARMA}(2,1)$ process, 18 observations missing $\}$ 


\begin{tabular}{|c|c|c|c|c|c|c|}
\hline Period & Error & Error Sq. & DELTA(T) & $\mathrm{Y}(\mathrm{T})$ & $\mathrm{Q}(\mathrm{T})$ & $\mathrm{Q}(\mathrm{T}) / \operatorname{Delta}(\mathrm{T})$ \\
\hline 1 & 0.003461 & $1.19774 \mathrm{E}-05$ & 0.009117 & 0.003461 & 0.000346 & 0.037960229 \\
\hline 2 & -0.01088 & 0.000118283 & 0.009293 & -0.00741 & -0.00078 & -0.083515869 \\
\hline 4 & 0.037384 & 0.001397559 & 0.012102 & 0.029969 & 0.00304 & 0.251190342 \\
\hline 5 & 0.004607 & $2.12199 \mathrm{E}-05$ & 0.011352 & 0.034576 & 0.003197 & 0.281575004 \\
\hline 6 & 0.000505 & $2.55418 \mathrm{E}-07$ & 0.010268 & 0.035081 & 0.002927 & 0.28511117 \\
\hline 7 & 0.006956 & $4.83825 \mathrm{E}-05$ & 0.009937 & 0.042037 & 0.00333 & 0.335154679 \\
\hline 8 & -0.00236 & $5.55462 \mathrm{E}-06$ & 0.009179 & 0.03968 & 0.002762 & 0.300871257 \\
\hline 9 & -0.00532 & 2.8292E-05 & 0.008793 & 0.034361 & 0.001954 & 0.222175965 \\
\hline 11 & 0.012393 & 0.000153581 & 0.009153 & 0.046754 & 0.002997 & 0.327494377 \\
\hline 12 & 0.002033 & $4.13327 \mathrm{E}-06$ & 0.008441 & 0.048787 & 0.002901 & 0.34369255 \\
\hline 13 & 0.00398 & $1.5839 \mathrm{E}-05$ & 0.007995 & 0.052766 & 0.003009 & 0.376364509 \\
\hline 15 & 0.015253 & 0.000232658 & 0.00872 & 0.06802 & 0.004233 & 0.485446046 \\
\hline 16 & -0.01524 & 0.000232206 & 0.009372 & 0.052781 & 0.002286 & 0.243927284 \\
\hline 19 & 0.009104 & $8.28845 E-05$ & 0.009345 & 0.061885 & 0.002968 & 0.317582217 \\
\hline 20 & 0.00647 & 4.18645E-05 & 0.009058 & 0.068356 & 0.003318 & 0.366329014 \\
\hline 21 & 0.00012 & 1.44053E-08 & 0.008164 & 0.068476 & 0.002998 & 0.367260585 \\
\hline 22 & 0.00027 & $7.28336 \mathrm{E}-08$ & 0.007375 & 0.068746 & 0.002726 & 0.369576099 \\
\hline 23 & -0.01056 & 0.000111617 & 0.007694 & 0.058181 & 0.001396 & 0.1815078 \\
\hline 25 & 0.007683 & 5.90307E-05 & 0.007693 & 0.065864 & 0.002025 & 0.263255888 \\
\hline 26 & -0.00989 & 9.79013E-05 & 0.007913 & 0.055969 & 0.000833 & 0.105293633 \\
\hline 27 & 0.010439 & 0.000108981 & 0.008165 & 0.066409 & 0.001794 & 0.219679968 \\
\hline 28 & -0.02039 & 0.000415762 & 0.009388 & 0.046018 & -0.00042 & -0.045229093 \\
\hline 29 & -0.00672 & 4.51353E-05 & 0.009121 & 0.0393 & -0.00105 & -0.115554942 \\
\hline 30 & -0.02406 & 0.000578855 & 0.010615 & 0.015241 & -0.00335 & -0.316021615 \\
\hline 32 & 0.005354 & $2.86662 \mathrm{E}-05$ & 0.010089 & 0.020595 & -0.00248 & -0.246180648 \\
\hline 33 & 0.000615 & 3.78798E-07 & 0.009141 & 0.02121 & -0.00217 & -0.237790487 \\
\hline 34 & -0.00067 & $4.44271 \mathrm{E}-07$ & 0.008294 & 0.020544 & -0.00202 & -0.243915926 \\
\hline 36 & -0.0031 & 9.61514E-06 & 0.007775 & 0.017443 & -0.00213 & -0.274071516 \\
\hline 37 & -0.00166 & 2.7402E-06 & 0.007163 & 0.015788 & -0.00208 & -0.290848252 \\
\hline 38 & -0.00379 & 1.43962E-05 & 0.006826 & 0.011993 & -0.00225 & -0.330267323 \\
\hline 40 & 0.003833 & 1.46939E-05 & 0.006527 & 0.015827 & -0.00165 & -0.252136811 \\
\hline 41 & -0.01115 & 0.000124313 & 0.006989 & 0.004677 & -0.0026 & -0.371445323 \\
\hline 43 & -0.00395 & 1.55935E-05 & 0.006685 & 0.000728 & -0.00273 & -0.408574936 \\
\hline 45 & -0.00212 & 4.49832E-06 & 0.006228 & -0.00139 & -0.00267 & -0.428714118 \\
\hline 46 & 0.014715 & 0.000216546 & 0.007077 & 0.013323 & -0.00093 & -0.131644031 \\
\hline 47 & -0.00382 & 1.4567E-05 & 0.006751 & 0.009506 & -0.00122 & -0.180735509 \\
\hline \multirow[t]{4}{*}{48} & -0.01757 & 0.000308753 & 0.007833 & -0.00807 & -0.00286 & -0.364512917 \\
\hline & SSE & 0.004567263 & & & & \\
\hline & SSE/35 & 0.000130493 & & & & \\
\hline & DELTA $(0)$ & 0.009745462 & & & & \\
\hline
\end{tabular}

Table A.6.1 Tracking Signal Analysis \{ARMA(2,2) process, 11 observations missing\} 


\begin{tabular}{|c|c|c|c|c|c|c|}
\hline eriod & Error & Error Sq. & $\operatorname{ELTA}(\mathrm{T})$ & $\overline{Y(T)}$ & $\mathrm{Q}(\mathrm{T})$ & \\
\hline 1 & 000961 & $23201 \mathrm{E}-07$ & 0.008628 & 000961 & $.60834 \mathrm{E}-05$ & 0.011135608 \\
\hline 2 & .01338 & 000178911 & 0.009103 & -0.01241 & -0.001251103 & -0.137435339 \\
\hline 4 & 034884 & 001216889 & 011681 & 022469 & 0.002362401 & 202238174 \\
\hline 5 & 002107 & 43738E-06 & .010724 & 024576 & 0.002336812 & 0.217908854 \\
\hline 6 & .00199 & 3.97847E-06 & 0.009851 & .022581 & 0.00190367 & 0.193248588 \\
\hline 7 & .004456 & 1.98537E-05 & 0.009311 & 0.027037 & 0.002158878 & 0.231853904 \\
\hline 8 & -0.00486 & $35887 \mathrm{E}-05$ & .008866 & 0.02218 & 308 & 0.164371907 \\
\hline 9 & .00782 & 6.11372E-05 & 0.008761 & 0.014361 & 0.000529674 & 0.060456626 \\
\hline 11 & 0.009893 & $9.7867 \mathrm{E}-05$ & 0.008874 & 0.024254 & 0.001465985 & 0.165192839 \\
\hline 12 & -0.00047 & $2.18047 \mathrm{E}-07$ & 0.008034 & 0.023787 & 0.001272691 & 0155 \\
\hline 13 & 00148 & 18987E-06 & .007378 & .025266 & 0.001293404 & 0.175299319 \\
\hline 15 & 0.012753 & 000162642 & 0.007916 & 0.03802 & 0.002439375 & 0.308167495 \\
\hline 16 & -0.01774 & 0.000314647 & 0.008898 & 0.020281 & 0.000421607 & 0.047382259 \\
\hline 19 & 006604 & $4.3614 \mathrm{E}-05$ & .008669 & 0.026885 & 0.001039856 & 0.119956469 \\
\hline 20 & 397 & -05 & 99 & 56 & 0.0013 & 0.162572825 \\
\hline 21 & -0.00238 & 5.66429E-06 & 0.007617 & 0.028476 & 61611 & 0.126247039 \\
\hline 22 & -0.00223 & 4.97345E-06 & 0.007078 & 0.026246 & 0.000642437 & 0.090762559 \\
\hline 23 & 01306 & 000170691 & 007677 & 013181 & 0.000728296 & -0.094868617 \\
\hline 25 & 183 & 05 & 28 & 18364 & 37151 & -0.018465331 \\
\hline 26 & -0.01239 & .000153624 & 0.007924 & 0.005969 & -0.001362887 & -0.171990233 \\
\hline 27 & 0.007939 & $30343 E-05$ & 0.007926 & 0.013909 & -0.000432657 & -0.054588907 \\
\hline 28 & 39 & 964 & 9422 & -0.00898 & .002678417 & -0.284267063 \\
\hline 30 & -0.01332 & .000177409 & 0.009812 & -0.0223 & -0.003742526 & -0.381426595 \\
\hline 32 & 0.00913 & 8.33587E-05 & .009744 & -0.01317 & -0.002455264 & -0.251983838 \\
\hline 33 & 001721 & $96173 \mathrm{E}-06$ & .008941 & -0.01145 & -0.00203764 & -0.227886815 \\
\hline 36 & 04785 & 2.28972E-05 & 0.008526 & -0.00666 & -0.001355366 & -0.158971871 \\
\hline 37 & 0.003384 & 1.14496E-05 & 0.008012 & -0.00328 & -0.000881456 & -0.110022327 \\
\hline 38 & -0.00237 & 5.60259E-06 & 0.007447 & -0.00565 & -0.001030009 & -0.138309109 \\
\hline 40 & 001478 & 1836E-06 & 00685 & -0.00417 & -0.000779238 & -0.113753919 \\
\hline 41 & -0.01238 & .000153172 & 0.007403 & -0.01655 & -0.001938943 & -0.261919687 \\
\hline 43 & -0.01018 & 0.000103628 & 0.007681 & -0.02673 & -0.002763029 & -0.359745359 \\
\hline 45 & -0.00879 & 7.72111E-05 & 0.007791 & -0.03551 & -0.003365425 & -0.431954234 \\
\hline 46 & 0.006777 & $.59242 \mathrm{E}-05$ & 0.00769 & -0.02874 & -0.002351208 & -0.305760006 \\
\hline \multirow[t]{4}{*}{48} & -0.01306 & 0.000170481 & 008226 & -0.1 & 771 & -0.4 \\
\hline & SSE & 0.003951757 & & & & \\
\hline & SSE/32 & 0.000123492 & & & & \\
\hline & $\overline{E L T A}(0)$ & $\overline{44}$ & & & & \\
\hline
\end{tabular}

Table A.6.2 Tracking Signal Analysis $\{\operatorname{ARMA}(2,2)$ process, 14 observations missing\} 


\begin{tabular}{|c|c|c|c|c|c|c|}
\hline Period & Error & Error Sq. & $\operatorname{DELTA}(T)$ & $\mathrm{Y}(\mathrm{T})$ & $\mathrm{Q}(\mathrm{T})$ & $\mathrm{Q}(\mathrm{T}) / \operatorname{Delta}(\mathrm{T})$ \\
\hline 1 & 0.003461 & 1.19774E-05 & 0.008948 & 0.003461 & 0.000346 & 0.038675292 \\
\hline 2 & -0.01088 & 0.000118283 & 0.009141 & -0.00741 & -0.00078 & -0.084901898 \\
\hline 4 & 0.037384 & 0.001397559 & 0.011965 & 0.029969 & 0.00304 & 0.254056642 \\
\hline 5 & 0.004607 & 2.12199E-05 & 0.01123 & 0.034576 & 0.003197 & 0.284656217 \\
\hline 6 & 0.000505 & $2.55418 \mathrm{E}-07$ & 0.010157 & 0.035081 & 0.002927 & 0.288215554 \\
\hline 7 & 0.006956 & $4.83825 \mathrm{E}-05$ & 0.009837 & 0.042037 & 0.00333 & 0.338545913 \\
\hline 8 & -0.00236 & 5.55462E-06 & 0.009089 & 0.03968 & 0.002762 & 0.303836656 \\
\hline 9 & -0.00532 & $2.8292 E-05$ & 0.008712 & 0.034361 & 0.001954 & 0.224232045 \\
\hline 11 & 0.012393 & 0.000153581 & 0.00908 & 0.046754 & 0.002997 & 0.33011146 \\
\hline 12 & 0.002033 & 4.13327E-06 & 0.008375 & 0.048787 & 0.002901 & 0.346372407 \\
\hline 13 & 0.00398 & $1.5839 \mathrm{E}-05$ & 0.007936 & 0.052766 & 0.003009 & 0.379151946 \\
\hline 15 & 0.015253 & 0.000232658 & 0.008668 & 0.06802 & 0.004233 & 0.488408661 \\
\hline 16 & -0.01524 & 0.000232206 & 0.009325 & 0.052781 & 0.002286 & 0.245172665 \\
\hline 19 & 0.009104 & 8.28845E-05 & 0.009303 & 0.061885 & 0.002968 & 0.319044963 \\
\hline 20 & 0.00647 & 4.18645E-05 & 0.009019 & 0.068356 & 0.003318 & 0.36789524 \\
\hline 21 & 0.00012 & 1.440 & 0.008129 & 0.068476 & 0.002998 & 0.368828476 \\
\hline 22 & 0.00027 & 7.28336E-08 & 0.007343 & 0.068746 & 0.002726 & 0.371148077 \\
\hline 23 & -0.01056 & 0.000 & 0.007666 & 0.058181 & 0.001396 & 0.182173433 \\
\hline 25 & 0.007683 & 5.90307E-05 & 0.007667 & 0.065864 & 0.002025 & 0.26412457 \\
\hline 26 & -0.00989 & 9.79013E-05 & 0.00789 & 0.055969 & 0.000833 & 0.105597506 \\
\hline 27 & 0.010439 & 0.000108981 & 0.008145 & 0.066409 & 0.001794 & 0.220232697 \\
\hline 28 & -0.02039 & 0.000415762 & 0.00937 & 0.046018 & -0.00042 & -0.045318127 \\
\hline 29 & -0.00672 & 4.51353E-05 & 0.009104 & 0.0393 & -0.00105 & -0.115765628 \\
\hline 30 & -0.02406 & 0.000578855 & 0.0106 & 0.015241 & -0.00335 & -0.31646702 \\
\hline 32 & 0.005354 & 2.86662E-05 & 0.010075 & 0.020595 & -0.00248 & -0.24650918 \\
\hline 33 & 0.000615 & 3.78798E-07 & 0.009129 & 0.02121 & -0.00217 & -0.238105683 \\
\hline 34 & -0.00067 & $4.44271 \mathrm{E}-07$ & 0.008283 & 0.020544 & -0.00202 & -0.24423664 \\
\hline 36 & -0.0031 & 9.61514E-06 & 0.007765 & 0.017443 & -0.00213 & -0.274417489 \\
\hline 37 & -0.00166 & $2.7402 \mathrm{E}-06$ & 0.007154 & 0.015788 & -0.00208 & -0.291206907 \\
\hline 38 & -0.00379 & 1.43962E-05 & 0.006818 & 0.011993 & -0.00225 & -0.330651923 \\
\hline 40 & 0.003833 & 1.46939E-05 & 0.006519 & 0.015827 & -0.00165 & -0.252413163 \\
\hline 41 & -0.01115 & 0.000124313 & 0.006982 & 0.004677 & -0.0026 & -0.371787433 \\
\hline 43 & -0.00395 & 1.55935E-05 & 0.006679 & 0.000728 & -0.00273 & -0.408928995 \\
\hline 45 & -0.00212 & 4.49832E-06 & 0.006223 & -0.00139 & -0.00267 & -0.429072969 \\
\hline 46 & 0.014715 & 0.000216546 & 0.007073 & 0.013323 & -0.00093 & -0.131731295 \\
\hline \multirow[t]{4}{*}{48} & -0.00489 & 2.39296E-05 & 0.006854 & 0.008431 & -0.00133 & -0.193696871 \\
\hline & SSE & 0.004267874 & & & & \\
\hline & SSE/34 & 0.000125526 & & & & \\
\hline & $\operatorname{DELTA}(0)$ & 0.009558169 & & & & \\
\hline
\end{tabular}

Table A.6.3 Tracking Signal Analysis $\{\operatorname{ARMA}(2,2)$ process, 12 observations missing\} 